\title{
The Inertia Operator on the Motivic Hall Algebra
}

\author{
Kai Behrend, Pooya Ronagh
}

October 8, 2018

\begin{abstract}
We study the action of the inertia operator on the motivic Hall algebra, and prove that it is diagonalizable. This leads to a filtration of the Hall algebra, whose associated graded algebra is commutative. In particular, the degree 1 subspace forms a Lie algebra, which we call the Lie algebra of virtually indecomposable elements, following Joyce. We prove that the integral of virtually indecomposable elements admits an Euler characteristic specialization. In order to take advantage of the fact that our inertia groups are unit groups in algebras, we introduce the notion of algebroid.
\end{abstract}

\section{Contents}

Introduction

Algebroids . . . . . . . . . . . . . . . 3

The motivic Hall algebra . . . . . . . . . . . . . . . 4

Example . . . . . . . . . . . . . . . 5

The spectrum of semi-simple inertia . . . . . . . . . 6

Results . . . . . . . . . . . . . . . . 8

Discussion . . . . . . . . . . . . . . . 11

Acknowledgements . . . . . . . . . . . . . 12

1 Linear algebraic stacks and algebroids $\mathbf{1 3}$

1.1 Algebraic stacks . . . . . . . . . . . . . . . . 13

Sheaves on algebraic stacks ............ . . . 14

Coherent sheaves . . . . . . . . . . . . . . . . . . 14

Representable sheaves of modules . . . . . . . . . . . . . . . . . 16

1.2 Linear algebraic stacks . . . . . . . . . . . . . . . 18

Examples . . . . . . . . . . . . . . . . . . . . 19

Substacks . . . . . . . . . . . . . . . 20

Fibered products . . . . . . . . . . . . . . . . . . . . . 20

Lack of locality . . . . . . . . . . . . . . . . 21

Special linear stacks . . . . . . . . . . . . . . 22

1.3 Finite type algebras . . . . . . . . . . . . . . . . . 23

Inertia representation . . . . . . . . . . . . . . . 23

Algebra bundles . . . . . . . . . . . . . . . . . . 24

Central idempotents . . . . . . . . . . . . . 25 
Primitive idempotents . . . . . . . . . . . . . . . 25

The degree stratification . . . . . . . . . . . . . . . . . . . . . . . . . . .

Semi-simple elements . . . . . . . . . . . . . . . . 27

The semi-simple centre . . . . . . . . . . . . . . 28

Permanence of rank and split rank . . . . . . . . . . . . . 29

Families of idempotents . . . . . . . . . . . . 30

Group of units . . . . . . . . . . . . . . 30

1.4 Algebroids . . . . . . . . . . . . . . . . . 31

Morphisms of algebroids . . . . . . . . . . . . . . . . . . . 34

Algebroid Inertia . . . . . . . . . . . . . . . 36

Idempotents and algebroids . . . . . . . . . . . . . . . . . . . . . . . . . . .

Algebroid representations . . . . . . . . . . . . . . . . . . . 40

Clear algebroids . . . . . . . . . . . . . . . . 41

2 The spectrum of semi-simple inertia 43

Stack functions . . . . . . . . . . . . . . . . . . 43

The filtration by split central rank . . . . . . . . . . 44

2.1 The idempotent operators $E_{r} \ldots \ldots \ldots \ldots$. . . . . . . . . . . . . . . .

2.2 The spectrum of semisimple Inertia . . . . . . . . . . . . 49

2.3 Graded structure of multiplication . . . . . . . . . . . 56

A combinatorial lemma . . . . . . . . . . . 57

3 The order filtration 59

The Hall algebra . . . . . . . . . . . . . . . . . . . . 59

3.1 Filtered structure of the Hall algebra . . . . . . . . . . . . 60

Analysis of $E_{p}\left(E_{n} * E_{m}\right) \ldots \ldots \ldots$. . . . . . . . 61

Decomposing $E_{p}\left(E_{n} * E_{m}\right) \ldots \ldots \ldots 1$

Proof of the main lemma . . . . . . . . . . . . 64

3.2 The semi-classical Hall algebra . . . . . . . . . . 65

3.3 Epsilon functions . . . . . . . . . . . . . . . . 65

Epsilons as logarithms . . . . . . . . . . . . . . . . . . . . . . .

Hopf algebra . . . . . . . . . . . . . . . . . 71

4 Integration 73

The vector bundle relations . . . . . . . . . . . . . 73

Regular motivic weights . . . . . . . . . . . . . 73

The integral . . . . . . . . . . . . . . . . . . . . . . . . . . . . . . . . . . .

4.1 The No Poles theorem . . . . . . . . . . . . . . . 74

4.2 The integral vs. the Hall product . . . . . . . . . 78

The $\Gamma$-indexed integral . . . . . . . . . . . . . . . . . 78

Semi-classical limit . . . . . . . . . . . . . . . 78

Appendix. Comparison with Joyce's virtual projections in an example 


\section{Introduction}

For simplicity, let us work over a field $k$. (Later, $k$ will be replaced by a noetherian ring $R$.)

Let $\mathfrak{M}$ be an abelian $k$-linear algebraic stack. Roughly, this means that $\mathfrak{M}$ is at the same time a $k$-linear abelian category with finite-dimensional hom-spaces, and an algebraic stack, locally of finite type over $k$. (The precise definition of linear algebraic stack is Definition 1.9. In the body of the paper we work with exact, instead of abelian categories, see the beginning of Section 3 .)

Examples we are interested in include

(i) $\mathfrak{M}=\mathfrak{C o h}_{Y}$, the moduli stack of coherent $\mathscr{O}_{Y}$-modules, for a projective $k$-variety $Y$,

(ii) $\mathfrak{M}=\mathfrak{R e p}_{Q}$, the moduli stack of representations of a quiver $Q$ on finite-dimensional $k$-vector spaces,

(iii) (the case $Y=Q=\operatorname{Spec} k) \mathfrak{M}=\mathfrak{V e c t}$, the stack of finite-dimensional $k$-vector spaces. In this case, $\mathfrak{V} \mathfrak{e c t}(S)$, for a $k$-scheme $S$, is the exact $\mathscr{O}(S)$-linear category of vector bundles over $S$, and $\mathfrak{V e c t}(k)$ is the abelian $k$-linear category of finite-dimensional $k$-vector spaces. As an algebraic stack, $\mathfrak{V} e c t$ is

$$
\mathfrak{V e c t}=\operatorname{Spec} k \amalg \mathrm{BGL}_{1} \amalg \mathrm{BGL}_{2} \amalg \ldots
$$

\section{Algebroids}

There is a canonical sheaf of algebras $\mathfrak{A} \rightarrow \mathfrak{M}$ over $\mathfrak{M}$. The set of sections of $\mathfrak{A}$ over the $S$-valued point $x$ of $\mathfrak{M}$ is the $\mathscr{O}(S)$-algebra $\mathfrak{A}_{x}=\operatorname{End}(x)$. For $\mathfrak{M}=\mathfrak{V e c t}$, the point $x$ is a vector bundle over $S$, and $\operatorname{End}(x)$ is the $\mathscr{O}(S)$-module of endomorphisms of $x$.

There is also a canonical isomorphism of group sheaves $\mathfrak{A}^{\times} \rightarrow I_{\mathfrak{M}}$ over $\mathfrak{M}$, where $I_{\mathfrak{M}}$ is the inertia stack of $\mathfrak{M}$. (Recall that the sections of $I_{\mathfrak{M}}$ over the $S$-valued point $x$ of $\mathfrak{M}$ are the automorphisms of $x$, in other words the units in the algebra of endomorphisms.)

We call a triple $\left(X, A_{X}, \iota\right)$ an algebroid (see Definition 1.38 and Remark 1.44), if $X$ is an algebraic stack, $A_{X} \rightarrow X$ is a representable sheaf of finite $\mathscr{O}$-algebras over $X$ (or finite type algebras, as we call them, see Definition 1.18), and $\iota: A_{X}^{\times} \rightarrow I_{X}$ is an open immersion of relative group schemes over $X$, making the canonical diagram

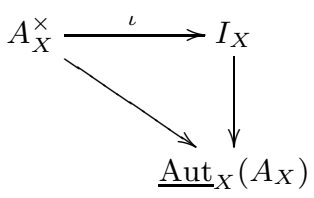

commute.

So $\mathfrak{M}$ with its canonical sheaf of algebras $\mathfrak{A}$ is an example of an algebroid. In this case, $\iota$ is an isomorphism, yielding what we call a strict algebroid.

Algebroids are generalizations of linear algebraic stacks (they are linear over their coarse moduli spaces, if they are strict). They are slightly 
more flexible, for example, schemes can be considered as algebroids in a canonical way. If the algebraic stack $X$ is the base of an algebroid, then the connected component $I_{X}^{\circ}$ of its inertia stack $I_{X}$ is the group of units in an algebra. This is the main significance of algebroids for us.

Just like algebraic stacks, algebroids form a 2-category, in which 2fibered products exist. Whenever $\left(X, A_{X}\right)$ is an algebroid, and $Y \rightarrow X$ is an inert morphism of algebraic stacks, i.e. $I_{Y}^{\circ}=\left.I_{X}^{\circ}\right|_{Y}$ (see Definition 1.47), the stack $Y$ is endowed with a natural structure of an algebroid via $\left.A_{X}\right|_{Y}$. Examples of inert morphisms include monomorphisms, and projections $Z \times X \rightarrow X$, for schemes $Z$. A locally closed immersion of algebroids $\left(Y, A_{Y}\right) \rightarrow\left(X, A_{X}\right)$ is a morphism where $Y \rightarrow X$ is a locally closed immersion of algebraic stacks, such that $A_{Y}=\left.A_{X}\right|_{Y}$. Every scheme $Z$ is an algebroid via the definition $A_{Z}=0_{Z}$.

A key observation is that if $(X, A)$ is an algebroid, then $\left(I_{X}, I_{A}\right)$ is another algebroid. In fact, $I_{A}$, the inertia stack of the stack $A$ (the total 'space' of the sheaf of algebras $A$ ), is equal to the subalgebra of $\left.A\right|_{I_{X}}$ fixed under its tautological automorphism. We call $\left(I_{X}, I_{A}\right)$ the inertia algebroid of $(X, A)$. It comes with a canonical morphism to $(X, A)$. There is also a semi-simple connected version of the algebroid inertia, denoted by $I^{\circ, s s}$.

\section{The motivic Hall algebra}

We define stack functions to be representable morphisms of algebroids $\left(X, A_{X}\right) \rightarrow(\mathfrak{M}, \mathfrak{A})$, where $X$ is of finite type. The Hall algebra $K(\mathfrak{M})$ of $\mathfrak{M}$ is the $\mathbb{Q}$-vector space on the isomorphism classes of stack functions modulo the scissor relations relative $\mathfrak{M}$ :

$$
[X \rightarrow \mathfrak{M}]=[Z \rightarrow X \rightarrow \mathfrak{M}]+[X \backslash Z \rightarrow X \rightarrow \mathfrak{M}]
$$

and the bundle relations relative $\mathfrak{M}$ :

$$
[Y \rightarrow X \rightarrow \mathfrak{M}]=[F \times X \rightarrow X \rightarrow \mathfrak{M}]
$$

Here $[X \rightarrow \mathfrak{M}]$ denotes the Hall algebra element defined by a stack function with base $X$. Also, $Z \rightarrow X$ is a closed immersion of algebroids, with open complement $X \backslash Z$, and $Y \rightarrow X$ is an inert fibre bundle (Definition 1.53) with special structure group and fibre $F$, all endowed with their canonical algebroid structure. (For strict algebroids, the bundle relations follow from the scissor relations.)

We have the following structures on the Hall algebra:

1. Module structure. Let $K(\operatorname{Var})$ denote the Grothendieck ring of varieties over $k$. We denote the motivic weight of the affine line by $q=\left[\mathbb{A}^{1}\right] \in K($ Var $)$. By $[Z] \cdot[X \rightarrow \mathfrak{M}]=[Z \times X \rightarrow \mathfrak{M}]$ we define a $K$ (Var)-module structure on $K(\mathfrak{M})$.

2. Multiplication. $\quad[X \rightarrow \mathfrak{M}] \cdot[Y \rightarrow \mathfrak{M}]=[X \times Y \rightarrow \mathfrak{M} \times \mathfrak{M} \stackrel{\oplus}{\longrightarrow}$ $\mathfrak{M}]$ defines a commutative multiplication on $K(\mathfrak{M})$, and $K(\mathfrak{M})$ is a $K$ (Var)-algebra with this multiplication.

3. Hall product. Using the stack of short exact sequences in $\mathfrak{M}$, we can define a Hall algebra product $[X \rightarrow \mathfrak{M}] *[Y \rightarrow \mathfrak{M}]$ on $K(\mathfrak{M})$. 
For details, see Section 3 The module $K(\mathfrak{M})$ is a $K$ (Var)-algebra also with respect to the Hall product.

4. Unit. The multiplicative unit with respect to both products is represented by $1=[\operatorname{Spec} k \stackrel{0}{\longrightarrow} \mathfrak{M}]$. Via this unit, we get an inclusion $K(\operatorname{Var}) \subset K(\mathfrak{M})$.

5. Inertia endomorphism. The algebroid inertia defines an operator $I: K(\mathfrak{M}) \rightarrow K(\mathfrak{M})$ via $I[X \rightarrow \mathfrak{M}]=\left[I_{X} \rightarrow X \rightarrow \mathfrak{M}\right]$. This inertia operator is linear over $K($ Var $)$, and multiplicative $I(x$. $y)=I(x) \cdot I(y)$, with respect to the commutative multiplication. The same facts hold for the connected semi-simple inertia operators $I^{\circ, s s}: K(\mathfrak{M}) \rightarrow K(\mathfrak{M})$.

There is also a 'non-representable' version of the Hall algebra, where we drop the representability requirement for stack functions, and simply define a stack function to be a morphism of algebroids $X \rightarrow \mathfrak{M}$, with $X$ of finite type. The representable Hall algebra is a subalgebra (with respect to both products) of the non-representable one. Our results on the diagonalizability of the various operators $I, I^{\circ, s s}, E_{n}$ hold true also in the non-representable Hall algebra, but the algebraic results on the structure of the Hall algebra need representability. For simplicity, we restrict ourselves therefore to the representable case.

Usually, when defining the Hall algebra of $\mathfrak{M}$, one requires the bundle relations also for non-inert morphisms. The connected inertia operator does not respect such relations, and we therefore do not include them.

\section{Example}

A stack function $X \rightarrow \mathfrak{V e c t}$ is the same thing as an algebroid $(X, A)$, together with a faithful representation.

Examples of stack functions with values in $\mathfrak{V e c t}$ include subalgebras $A \subset M_{n \times n}$. The elements of $K(\mathfrak{V e c t})$ defined by $A \subset M_{n \times n}$ and $B \subset$ $M_{n \times n}$ are equal if and only if $A$ and $B$ are conjugate in $M_{n \times n}$.

The subalgebra $U$ of $K(\mathfrak{V} \mathfrak{e c t})$ with respect to the Hall product, generated by the $[n]=\left[B \mathrm{GL}_{n} \rightarrow \mathfrak{V e c t}\right]$ is free on these elements $[n]$, for $n>0$, as a unitary $\mathbb{Q}$-algebra. In the literature, $U$ is known as the Hopf algebra of non-commutative symmetric functions, see [6. Example $4.1(\mathrm{~F})]$.

(If we add the (non-inert) vector bundle relations relative $\mathfrak{V e c t}$, see, e.g. 5], we get

$$
\left[\lambda_{1}\right] * \ldots *\left[\lambda_{r}\right]=\frac{\left[\mathrm{GL}_{n}\right]}{[P(\lambda)]}[n]=\left(\begin{array}{c}
n \\
\lambda_{1} \ldots \lambda_{n}
\end{array}\right)_{q}[n] .
$$

Here $n=\sum \lambda_{i}$, and $\left(\begin{array}{c}n \\ \lambda_{1} \ldots \lambda_{n}\end{array}\right)_{q}$ denotes the $q$-deformed multinomial coefficient, which gives the motivic weight of the flag variety of type $\lambda$. We have also denoted the parabolic subgroup of $\mathrm{GL}_{n}$ of type $\lambda$ by $P(\lambda)$. Hence the $\mathbb{Q}$-algebra obtained by dividing $U$ by the vector bundle relations is the commutative polynomial algebra over $\mathbb{Q}$, on the symbols $[1],[2],[3], \ldots$. This is the Hopf algebra of symmetric functions.) 


\section{The spectrum of semi-simple inertia}

The main point of this work is to study the spectral theory of the semisimple inertia operator $I^{\circ, s s}$ on $K(\mathfrak{M})$.

Before announcing our results, let us do a few sample calculations. They contain some of the central ideas of this paper. Only strict algebroids will occur, so we write $I^{s s}$ instead of $I^{\circ, s s}$.

We consider $\mathfrak{M}=\mathfrak{V e c t}$. The linear stack of line bundles defines the stack function $\left[\mathrm{BGL}_{1} \rightarrow \mathfrak{V} \mathfrak{e c t}\right] \in K(\mathfrak{V} \mathfrak{e c t})$. We have

$$
\begin{aligned}
I^{s s}\left[\mathrm{BGL}_{1} \rightarrow \mathfrak{V} \mathfrak{e c t}\right] & =\left[\mathrm{GL}_{1}^{s s} \times \mathrm{BGL}_{1} \rightarrow \mathfrak{V} \mathfrak{e c t}\right] \\
& =\left[\mathrm{GL}_{1} \times \mathrm{BGL}_{1} \rightarrow \mathfrak{V e c t}\right] \\
& =(q-1)\left[\mathrm{BGL}_{1} \rightarrow \mathfrak{V e c t}\right] .
\end{aligned}
$$

This proves that $\left[\mathrm{BGL}_{1} \rightarrow \mathfrak{V} \mathfrak{e c t}\right]$ is an eigenvector of $I^{s s}$, with corresponding eigenvalue $(q-1) \in K(\operatorname{Var})$.

Because $I^{s s}$ is an algebra morphism with respect to the commutative product, it immediately follows that every $(q-1)^{r}$, for $r \geq 0$ is and eigenvalue of $I^{s s}$, with corresponding eigenvector $\left[\mathrm{BGL}_{1}^{r} \rightarrow \mathrm{BGL}_{n} \rightarrow \mathfrak{V e c t}\right] \in$ $K(\mathfrak{V e c t})$.

These are not the only eigenvalues of $I^{s s}$. In fact, let us consider the stack function of all rank 2 vector bundles $\left[\mathrm{BGL}_{2} \rightarrow \mathfrak{V} \mathfrak{e c t}\right]$. Recall that the inertia stack of $\mathrm{BGL}_{2}$ is the quotient stack $\mathrm{GL}_{2} /{ }_{a d} \mathrm{GL}_{2}$, where $\mathrm{GL}_{2}$ acts on itself by the adjoint action. The semi-simple part of $\mathrm{GL}_{2}$ decomposes as $\mathrm{GL}_{2}^{e q} \sqcup \mathrm{GL}_{2}^{n e q}$, according to whether the two eigenvalues of an element of $\mathrm{GL}_{2}$ are equal or not equal. By the scissor relations we have,

$$
\begin{aligned}
I^{s s}\left[\mathrm{BGL}_{2}\right] & =\left[\mathrm{GL}_{2}^{\mathrm{eq}} /{ }_{a d} \mathrm{GL}_{2}\right]+\left[\mathrm{GL}_{2}^{\text {neq }} /{ }_{a d} \mathrm{GL}_{2}\right] \\
& =\left[\Delta \times \mathrm{BGL}_{2}\right]+\left[T^{*} / a d N\right] \\
& =(q-1)\left[\mathrm{BGL}_{2}\right]+x
\end{aligned}
$$

Here $\Delta$ is the one-parameter subgroup of scalar matrices, and $T$ is the maximal torus of diagonal matrices in $\mathrm{GL}_{2}$. Further notation: $T^{*}=T \backslash \Delta$, $N$ is the normalizer of $T$ in $\mathrm{GL}_{2}$, and $x=\left[T^{*} /{ }_{a d} N\right]$.

Next, we calculate $I^{s s} x$. In fact, we have $I_{T^{*} / N}^{s s}=I_{T^{*} / N}=\left(T^{*} \times T\right) / N$, by the 'stabilizer formula' for the inertia stack of a quotient stack

$$
I_{Y / G}=\{(y, g) \in Y \times G \mid y g=y\} / G .
$$

We note that $N=T \rtimes \mathbb{Z}_{2}$ acts on $T^{*} \times T$ diagonally, via its quotient $\mathbb{Z}_{2}$ by swapping the entries of $T$. We embed $T$ into $\mathbb{A}^{2}$ equivariantly with respect to $\mathbb{Z}_{2}$, and then decompose $\mathbb{A}^{2}$ as $T \sqcup\left(\mathrm{GL}_{1} \times 0\right) \sqcup\left(0 \times \mathrm{GL}_{1}\right) \sqcup(0,0)$. This gives

$$
\begin{aligned}
& {\left[\left(T^{*} \times \mathbb{A}^{2}\right) / N\right]} \\
& =\left[\left(T^{*} \times T\right) / N\right]+\left[T^{*} \times\left(\mathrm{GL}_{1} \times 0 \sqcup 0 \times \mathrm{GL}_{1}\right) / N\right]+\left[T^{*} \times(0,0) / N\right] .
\end{aligned}
$$


We have a pullback diagram of algebroids

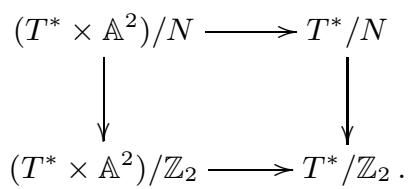

It shows that the vector bundle $\left(T^{*} \times \mathbb{A}^{2}\right) / N \rightarrow T^{*} / N$ is a pullback of the vector bundle $\left(T^{*} \times \mathbb{A}^{2}\right) / \mathbb{Z}_{2} \rightarrow T^{*} / \mathbb{Z}_{2}$. The latter is a vector bundle over a scheme, and is therefore Zariski-locally trivial by Hilbert's Theorem 90. The same is then true for any pullback bundle. Hence, we conclude that

$$
\left[\left(T^{*} \times \mathbb{A}^{2}\right) / N\right]=q^{2}\left[T^{*} / N\right],
$$

using only the scissor relations. So from (1) we conclude that

$$
q^{2} x=I^{s s} x+(q-1)\left[T^{*} / T\right]+x,
$$

which we solve for $I^{s s} x$ to get:

$$
I^{s s} x=\left(q^{2}-1\right) x-(q-1)^{2}(q-2)[B T] .
$$

We already know that $I^{s s}[B T]=(q-1)^{2}[B T]$, and so we conclude that $\left[\mathrm{BGL}_{2}\right], x$ and $[B T]$ generate an $I^{s s}$-invariant subspace of $K(\mathfrak{V e c t})$, and the matrix of $I^{s s}$ on this subspace is

$$
\left(\begin{array}{ccc}
q-1 & 0 & 0 \\
1 & q^{2}-1 & 0 \\
0 & -(q-1)^{2}(q-2) & (q-1)^{2}
\end{array}\right)
$$

This matrix is lower triangular, with distinct scalars on the diagonal, and is therefore diagonalizable over the field $\mathbb{Q}(q)$. So on this subspace, $I^{s s}$ is diagonalizable, with eigenvalues $(q-1),\left(q^{2}-1\right)$, and $(q-1)^{2}$. If we decompose $\left[\mathrm{BGL}_{2}\right]$ as a sum of eigenvectors, we get the eigenvectors

$$
\begin{array}{lll}
{\left[\mathrm{BGL}_{2}\right]-\frac{1}{q(q-1)} x-\frac{1}{q}[B T]} & \text { with eigenvalue } & (q-1), \\
\frac{1}{q(q-1)} x-\frac{q-2}{2 q}[B T] & \text { with eigenvalue } & \left(q^{2}-1\right), \\
\frac{1}{2}[B T] & \text { with eigenvalue } & (q-1)^{2} .
\end{array}
$$

A very important observation is that when we add together the eigencomponents whose eigenvalues have the same order of vanishing at $q=1$, we get coefficients in $\mathbb{Q}$, instead of $\mathbb{Q}(q)$. In the above example, we add together the components of $\left[\mathrm{BGL}_{2}\right]$ with eigenvalues $(q-1)$ and $\left(q^{2}-1\right)$ to obtain $\left[\mathrm{BGL}_{2}\right]-\frac{1}{2}[B T]$.

Another important observation is that diagonalizing $I^{s s}$ does not, despite appearances, require us to invert $(q-1)$. In fact, the algebroid $x$ appearing in the above argument is divisible by $(q-1)$, although the quotient is not a strict algebroid any longer. This is, in fact, the reason for considering non-strict algebroids at all.

(In the above calculations, we have suppressed the algebra part $A$ of the various algebroids $(X, A)$. We leave it to the reader to supply the natural algebra for each algebroid mentioned.) 


\section{Results}

We now summarize the main results of this paper.

Theorem 1 (Diagonalizability of $\boldsymbol{I}^{\circ, s s}$ ). The operator $I^{\circ, s s}$ on

$$
K(\mathfrak{M})(q)=K(\mathfrak{M}) \otimes_{\mathbb{Q}[u]} \mathbb{Q}(q)
$$

is diagonalizable, the eigenvalues are indexed by partitions $\lambda$, and the eigenvalue corresponding to the partition $\lambda$ is the cyclotomic polynomial

$$
\mathscr{Q}(\lambda)=\prod\left(q^{\lambda_{i}}-1\right) .
$$

In other words, we have a direct sum decomposition

$$
K(\mathfrak{M})(q)=\bigoplus_{\lambda} K^{\lambda}(\mathfrak{M})
$$

into subspaces invariant under $I^{s s}$, and $\left.I^{s s}\right|_{K^{\lambda}(\mathfrak{M})}$ is multiplication by Q $(\lambda)$.

The same theorem holds for the operator $I^{s s}$ in the context of strict algebroids. We also prove a stronger version avoiding denominators divisible by $(q-1)$, but this version only works for algebroids.

The proof of this theorem is a generalization of the above sample calculation for the stack of rank 2 vector bundles. One goal of Section 1 of the paper is to set up the necessary notation.

Theorem 2 (Graded structure of $\boldsymbol{K}(\mathfrak{M}))$. There is a direct sum decomposition

$$
K(\mathfrak{M})=\bigoplus_{r \geq 0} K^{r}(\mathfrak{M})
$$

such that

$$
K^{r}(\mathfrak{M})(q)=\bigoplus_{\operatorname{ord}_{q=1}(\lambda)=r} K^{\lambda}(\mathfrak{M}) .
$$

Moreover, the commutative product is graded with respect to (2).

Again, the same theorem holds in the context of strict algebroids.

The fact about the gradedness of the commutative product is expected from the fact that the semi-simple inertia respects the commutative product (it follows from this fact over $\mathbb{Q}(q)$, but is true over $\mathbb{Q}$ ).

Geometrically, the descending filtration $K^{\geq r}(\mathfrak{M})$ induced by the grading (2) can be described as follows: $K^{\geq r}(\mathfrak{M})$ is the $\mathbb{Q}$-span of all stack functions $[X \rightarrow \mathfrak{M}]$, for which the algebra of global sections $\Gamma\left(X, A_{X}\right)$ admits at least $r$ orthogonal non-zero central idempotents, where $A_{X}$ is the algebra of the algebroid $\left(X, A_{X}\right)$.

The direct summands $K^{r}(\mathfrak{M})$, are the common eigenspaces of the family of commuting operators $\left(E_{n}\right)_{n \geq 0}$, where $E_{n}(X)$ is the stack of decompositions of $1 \in A_{X}$ into a sum of $n$ orthogonal labelled idempotents. The eigenvalues of the operators $E_{n}$ are integers, and the whole family of operators $\left(E_{n}\right)$ is diagonalizable over $\mathbb{Q}$. The proof of this fact proceeds 
by proving that the $\left(E_{n}\right)$ preserve the descending filtration described geometrically above, and have distinct integer diagonal entries.

It turns out that the ascending filtration $K^{\leq n}(\mathfrak{M})$ associated to the grading in the above theorem can be described as

$$
K^{\leq n}(\mathfrak{M})=\operatorname{ker} E_{n+1} .
$$

Let us also point out that

$$
K^{0}(\mathfrak{M})=K(\mathrm{DM})
$$

and

$$
K^{0}(\mathfrak{M})=K(\operatorname{Var}),
$$

in the context of strict algebroids.

If we denote by $\pi_{r}: K(\mathfrak{M}) \rightarrow K(\mathfrak{M})$ the projection operator onto the summand $K^{r}(\mathfrak{M})$, and form the generating series $\pi_{t}=\sum_{r \geq 0} \pi_{r} t^{r}$, then we have

$$
\pi_{t}=\sum_{n \geq 0}\left(\begin{array}{l}
t \\
n
\end{array}\right) E_{n} .
$$

All the above results could be proved for pairs $(X, A)$ of algebraic stacks $X$ endowed with finite type algebras $A$, instead of algebroids or strict algebroids. One simply replaces $I^{\circ, s s}$ by $A^{\times, s s}$.

Theorem 3 (Filtered nature of the Hall algebra). The Hall product is filtered with respect to the filtration $K^{\leq r}(\mathfrak{M})$, induced by the grading (2). Moreover, for the associated graded algebra we have

$$
\operatorname{gr}(K(\mathfrak{M}), *)=(K(\mathfrak{M}), \cdot) \text {. }
$$

In other words, if $x \in K^{\leq r}(\mathfrak{M})$ and $y \in K^{\leq s}(\mathfrak{M})$, then $x * y \in K^{\leq r+s}(\mathfrak{M})$, and

$$
x * y \equiv x \cdot y \quad \bmod K^{\leq r+s-1}(\mathfrak{M}) .
$$

The proof of this theorem uses not much more than some simple combinatorics involving relabelling of direct sum decompositions, and compatibilities between direct sum decompositions of short exact sequences and splittings of short exact sequences.

The theorem implies that the one parameter family of algebras $(\mathscr{K}(\mathfrak{M}), *)$ given by the Rees construction

$$
\mathscr{K}(\mathfrak{M})=\bigoplus_{n \geq 0} t^{n} K^{\leq n}(\mathfrak{M})
$$

is a deformation quantization of (i.e., a one-parameter flat family of algebras with special fibre) the commutative algebra $(K(\mathfrak{M}), \cdot)$. Hence the graded algebra $(K(\mathfrak{M}), \cdot)$ inherits a Poisson bracket $\{$,$\} of degree -1$. In particular, $K^{1}(\mathfrak{M})$ is a Lie algebra, and it turns out that the Lie bracket on $K^{1}(\mathfrak{M})$ is equal to the commutator bracket associated to $*$.

Following Joyce [10, we call $K^{1}(\mathfrak{M})$ the Lie algebra of virtually indecomposable elements of $K(\mathfrak{M})$, with the notation $K^{\text {vir }}(\mathfrak{M})=K^{1}(\mathfrak{M})$. 
We denote the projection onto $K^{\text {vir }}(\mathfrak{M})$ by $\pi^{\text {vir }}$. With this notation, we have, as a special case of (3),

$$
\pi^{v i r}=\sum_{n>0} \frac{(-1)^{n+1}}{n} E_{n}
$$

In terms of eigenspaces of semi-simple inertia, we have

$$
K^{\text {vir }}(\mathfrak{M})(q)=K^{(q-1)}(\mathfrak{M}) \oplus K^{\left(q^{2}-1\right)}(\mathfrak{M}) \oplus K^{\left(q^{3}-1\right)}(\mathfrak{M}) \oplus \ldots
$$

Theorem 4 (Hall algebra logarithms). Let $\mathfrak{N} \subset \mathfrak{M}$ be a 'small enough' substack, closed under extensions and direct summands, and not intersect$\operatorname{ing} \operatorname{Spec} k \stackrel{0}{\longrightarrow} \mathfrak{M}$. Then

$$
\varepsilon_{t}[\mathfrak{N}]=\sum_{n \geq 0}\left(\begin{array}{c}
t \\
n
\end{array}\right)[\mathfrak{N}]^{* n} \in \hat{\mathscr{K}}(\mathfrak{M})_{+} .
$$

In particular, the $*$-logarithm

$$
\varepsilon[\mathfrak{N}]=\sum_{n \geq 1} \frac{(-1)^{n+1}}{n}[\mathfrak{N}]^{* n} \in \hat{K}^{v i r}(\mathfrak{M})_{+},
$$

is virtually indecomposable.

For the precise definition of 'small enough', see Section 3.3 For example, if $\mathfrak{M}=\mathfrak{C o h}_{Y}$ for a curve $Y$, we could take $\mathfrak{N}$ to consists of all non-zero semi-stable vector bundles of a fixed slope. Since $\mathfrak{N}$ is typically not of finite type, to make sense of $[\mathfrak{N}]$, we have to pass to a certain completion $\hat{K}(\mathfrak{M})_{+}$of $K(\mathfrak{M})$. See Section 3.3 for details.

Theorem 5 (No poles theorem). Let $K(\mathrm{St})$ be the $K$-ring of algebraic stacks, modulo all bundle relations with special structure group (inert or not). Consider the map

$$
\begin{aligned}
\int: K(\mathfrak{M}) & \longrightarrow K(\mathrm{St}) \\
{[(X, A) \rightarrow(\mathfrak{M}, \mathfrak{A})] } & \longmapsto[X],
\end{aligned}
$$

which forgets the structure map to $(\mathfrak{M}, \mathfrak{A})$, and the algebroid structure over the stack $X$. If $x \in K^{\leq r}(\mathfrak{M})$, then $(q-1)^{r} \int x$ is a regular element of $K(\mathrm{St})$, i.e., under the identification

$$
K(\mathrm{St})=K(\operatorname{Var})\left[\frac{1}{q}, \frac{1}{q-1}, \frac{1}{q^{2}-1}, \ldots\right],
$$

it can be written with a denominator that does not vanish at $q=1$.

Moreover, suppose we have a grading monoid $\Gamma$ for $\mathfrak{M}$ :

$$
\mathfrak{M}=\coprod_{\gamma \in \Gamma} \mathfrak{M}_{\gamma}
$$

We say that $x$ has 'degree' $\gamma$ if $x \in \mathfrak{M}_{\gamma}$. We need $\Gamma$ to be endowed with a $\mathbb{Z}$-valued bilinear form $\chi$, such that for every object $x$ in $\mathfrak{M}_{\gamma}$, and $y$ in $\mathfrak{M}_{\beta}$, 
(i) every extension of $y$ by $x$ is in $\mathfrak{M}_{\beta+\gamma}$,

(ii) the stack of extensions of $y$ by $x$ is the quotient of a vector space $E_{1}$ by another vector space $E_{0}$, acting trivially, such that $\operatorname{dim} E_{0}-$ $\operatorname{dim} E_{1}=\chi(\beta, \gamma)$.

For the precise assumptions, see Section 4.2. They are satisfied if $\mathfrak{M}=$ $\mathfrak{R e p}{ }_{Q}$, or if $\mathfrak{M}=\mathfrak{C o h}_{Y}$ and $Y$ is a smooth curve, or more generally, if $\mathfrak{M}$ is hereditary.

Then we have a commutative diagram

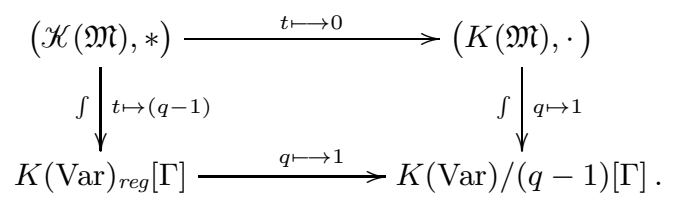

Here we use the $\Gamma$-graded integral, which is essentially a generating function, indexed by $\Gamma$, of the integrals of the components of degree $\gamma \in \Gamma$ of a given stack function.

The upper horizontal arrow in this diagram is the specialization map, which exists because of Theorem 3. The left vertical arrow exists by the 'No Poles Theorem' [5] It is a standard fact, that this map is an algebra morphism, i.e., respects the $*$-product, if the target $K(\operatorname{Var})_{\text {reg }}[\Gamma]$ is endowed with its q-deformed product twisted by $\chi$. It is a formal consequence of the commutativity of this diagram that the right vertical map is a morphism of Poisson algebras, if the target $K(\operatorname{Var}) /(q-1)[\Gamma]$ is endowed with its bracket induced by $\chi$.

In particular, we deduce that

$$
\begin{aligned}
& K^{\text {vir }}(\mathfrak{M}) \longrightarrow K(\operatorname{Var}) /(q-1)[\Gamma] \\
&\left.x_{\gamma} \longmapsto\left((q-1) \int x_{\gamma}\right)\right|_{q=1} u^{\gamma}=\operatorname{res}_{q=1}\left(\int x_{\gamma}\right) u^{\gamma}
\end{aligned}
$$

is a morphism of Lie algebras.

The proof of the No Poles Theorem combines the above results about the diagonalizability of $I^{\circ, s s}$, especially in its form avoiding denominators divisible by $(q-1)$, with the result that for an algebroid $(X, A)$, the stack $I_{X}^{\circ, s s}$ has regular motivic weight, i.e.,

$$
\left[I_{X}^{\circ, s s}\right] \in K(\mathrm{St})_{r e g} .
$$

We think of this as a motivic version of Burnside's lemma. The more natural looking conjecture that for an algebraic stack $X$, the motivic weight of $I_{X}$ is contained in $K(\operatorname{Var}) \subset K(\mathrm{St})$ is most likely false.

\section{Discussion}

To produce counting invariants for $\mathfrak{M}$, we need to look for subcategories $\mathfrak{N} \subset \mathfrak{M}$, to which we can apply Theorem 4 , giving us virtually indecomposable elements $\varepsilon[\mathfrak{N}]$, to which we can apply the integral (44), yielding generating functions with coefficients in $K(\operatorname{Var}) /(q-1)$. We can apply the Euler characteristic to these elements of $K(\operatorname{Var}) /(q-1)$ to obtain rational numbers. In the hereditary case, the fact that (4) is a morphism 
of Lie algebras, gives the relations among generating functions one is interested in. This leads to wall-crossing formulas, and other results. For details we refer to the works of Joyce, Joyce-Song, and many others.

To deal with the Calabi-Yau 3 case one needs to insert the correct motivic vanishing cycle weights, to define the integral. This is done by Joyce and Song in 12 .

The work of Joyce on configurations in abelian categories contains results which correspond to ours, but his definitions are more ad-hoc. In fact, one reason for writing the present article is to give a more conceptual treatment of Joyce's results. We do not prove that our notion of 'virtual indecomposable object' coincides with Joyce's (except for in the case of $\mathfrak{M}=\mathfrak{V} \mathfrak{e c t}$, see the appendix), but instead prove that our notion has the same properties as Joyce's and is just as useful. (Of course, the counting invariants we obtain are the same as the ones obtained by Joyce, as they do not depend on the definition of virtual indecomposable object.)

We think of the Lie algebra $K^{\text {vir }}(\mathfrak{M})$ as an analogue of the Lie algebra of primitive elements in a cocommutative Hopf algebra. In fact, one may ask whether $(K(\mathfrak{M}), *)$ is equal to the universal enveloping algebra of the Lie algebra $K^{\text {vir }}(\mathfrak{M})$. To deduce such a statement from structure theorems for Hopf algebras, one would need to enhance $K(\mathfrak{M})$ to a cocommutative Hopf algebra. We have not been able to construct the necessary coproduct. We view the family of operators $\left(E_{n}\right)$ as somewhat of a replacement. It lets us prove at least some of the results expected of a cocommutative Hopf algebra, in particular Theorems 3 and 4 .

\section{Acknowledgements}

The idea to consider the inertia stack as an operator on $K$-groups of stacks and motivic Hall algebras, and to study its eigenspace decomposition to understand Joyce's work in a more conceptual fashion is due to Tom Bridgeland. In particular, the conjecture that the semi-simple inertia operator is diagonalizable is due to him. We would like to thank Tom Bridgeland for sharing his ideas with us. We would also like to thank Dominic Joyce and Arend Bayer for fruitful discussions. 


\section{Linear algebraic stacks and algebroids}

\subsection{Algebraic stacks}

Let us briefly summarize our conventions about algebraic stacks.

We choose a noetherian base ring $R$ (commutative and with unit), and we fix our base category $\mathscr{S}$ to be the category of $R$-schemes, endowed with the étale topology. Over $\mathscr{S}$ we have a canonical sheaf of $R$-algebras $\mathscr{O}_{\mathscr{S}}$, it is represented by $\mathbb{A}^{1}=\mathbb{A}_{\text {Spec } R}^{1}$, and called the structure sheaf.

We will assume our algebraic stacks to be locally of finite type. Thus, an algebraic stack is a stack over the site $\mathscr{I}$, which admits a presentation by a smooth groupoid $X_{1} \rightrightarrows X_{0}$, where $X_{0}$ and $X_{1}$ are algebraic spaces, locally of finite type over $R$, the source and target morphisms $s, t: X_{1} \rightarrow$ $X_{0}$ are smooth, and the diagonal $X_{1} \rightarrow X_{0} \times X_{0}$ is of finite type. In fact, all algebraic stacks we encounter will have affine diagonal.

By a stratification of an algebraic stack $X$, we mean a morphism of algebraic stacks $X^{\prime} \rightarrow X$, which is a surjective monomorphism, and which admits a finite decomposition $X^{\prime}=\coprod_{i} X_{i}$, such that every $X_{i} \rightarrow X$ is a locally closed embedding of algebraic stacks.

If $G$ is an algebraic group acting on the algebraic space $X$, we will denote the quotient stack by $X / G$, because we fear the more common notation $[X / G]$ would lead to confusion with the notation for elements of various $K$-groups of schemes and stacks.

Suppose $G \rightarrow X$ is a relative group scheme over the stack $X$. The connected component of $G$, notation $G^{\circ}$, is the subsheaf of $G$, defined by requiring a section $g \in G(S)$ to factor through $G^{\circ}(S)$, if and only if for all points (equivalently geometric points) $s$ of $S$, we have $g(s) \in G_{s}^{\circ}$. If $G \rightarrow X$ is smooth, the connected component $G^{\circ} \subset G$ is represented by an open substack of $G$, which is a smooth group scheme with geometrically connected fibers over $X$. (See [2], Exposé $\mathrm{VI}_{\mathrm{B}}$, Théoreme 3.10.)

If the inertia stack $I_{X}$ of an algebraic stack $X$ is smooth over $X$, the connected component $I_{X}^{\circ}$ exists. We can apply the rigidification construction (see for example [1], Appendix) to $I_{X}^{\circ} \subset I_{X}$, and obtain a (uniquely determined) Deligne-Mumford stack $\frac{X}{X}$, together with a morphism $X \rightarrow \bar{X}$, making $X$ a connected gerbe over $\bar{X}$, (which means that the relative inertia of $X$ over $\bar{X}$ has connected fibres). The structure morphism $X \rightarrow \bar{X}$ is smooth.

A gerbe $X \rightarrow \bar{X}$ is an isotrivial gerbe, if it admits a section over a finite étale $\bar{X}$-stack. If $X \rightarrow \bar{X}$ is a smooth gerbe over a Deligne-Mumford stack, there exists a stratification $\bar{X}^{\prime} \rightarrow \bar{X}$, such that the restriction of the gerbe $X$ to each piece of $\bar{X}^{\prime}$ is isotrivial. (This follows from the fact that a quasifinite morphism of Deligne-Mumford stacks is generically finite. This, in turn, follows from Zariski's main theorem [15, Section 16].)

Let us also remark that every Deligne-Mumford stack admits a stratification by integral normal Deligne-Mumford stacks, although we do not use this fact. 


\section{Sheaves on algebraic stacks}

We need to clarify the notions of vector bundle, coherent sheaf, and representable sheaf of $\mathscr{O}_{X}$-modules, and how they relate to each other.

In particular, an algebraic stack $X$ is a fibered category $X \rightarrow \mathscr{S}$. The category $X$ inherits a topology from $\mathscr{S}$, called the étale topology, and $X$ endowed with this topology is the big étale site of $X$. Sheaves over $X$ are by definition sheaves on this big étale site. For example, $\mathscr{O}_{\mathscr{S}}$ induces a sheaf of $R$-algebras on $X$, which is denoted by $\mathscr{O}_{X}$, and called the structure sheaf of $X$. It is represented by $\mathbb{A}_{X}^{1}$.

A sheaf $\mathscr{F}$ over $X$ induces for every object $x$ of $X$ lying over the object $U$ of $\mathscr{S}$ a sheaf on the usual (small) étale site $U_{\text {ét }}$ of the scheme $U$, denoted $\mathscr{F}_{U}$. Moreover, for every morphism $\alpha: y \rightarrow x$ lying over $f: V \rightarrow U$, we obtain a morphism of sheaves $\alpha^{*}: f^{-1}\left(\mathscr{F}_{U}\right) \rightarrow \mathscr{F}_{V}$. (The $\alpha^{*}$ satisfy an obvious cocycle condition, and the condition that they are isomorphisms if $f$ is étale.) For example, the structure sheaf $\mathscr{O}_{X}$ induces the structure sheaf on $U_{\text {ét }}$, for every such $x / U$. The data of the small étale sheaves $\mathscr{F}_{U}$, together with the compatibility morphisms $\alpha^{*}$, satisfying the two parenthetical conditions, is equivalent to the data defining $\mathscr{F}_{\text {(see }}$ 3, Exp. IV, 4.10]). The functor $\mathscr{F} \mapsto \mathscr{F}_{U}$ is the sheaf pullback morphism of a morphism of sites $U_{\text {ét }} \rightarrow X$, from the small étale site of $U$ to the big étale site of $X$. In particular, $\mathscr{F} \mapsto \mathscr{F}_{U}$ is exact. Both $U_{\text {ét }}$ and $X$ are ringed sites, and $\mathscr{F} \mapsto \mathscr{F}_{U}$ is also the sheaf of modules pullback of the morphism of ringed sites $U_{\text {ét }} \rightarrow X$. Therefore, the functor $\mathscr{F} \rightarrow \mathscr{F}_{U}$ is also exact when considered as a functor from the category of big sheaves of $\mathscr{O}_{X}$-modules to the category of small sheaves of $\mathscr{O}_{U_{\text {ét }}}$-modules.

If $\mathscr{F}$ and $\mathscr{G}$ are sheaves of $\mathscr{O}_{X}$-modules, then $\mathscr{H}$ om $(\mathscr{F}, \mathscr{G})$ is again a sheaf of $\mathscr{O}_{X}$-modules. In particular, for a sheaf of $\mathscr{O}_{X}$-modules, we have the dual $\mathscr{F}^{\vee}=\mathscr{H} \operatorname{lom}\left(\mathscr{F}, \mathscr{O}_{X}\right)$.

Note that, in general, the natural homomorphism $\mathscr{H}$ om $(\mathscr{F}, \mathscr{G})_{U} \rightarrow$ $\mathscr{H} \operatorname{lom}\left(\mathscr{F}_{U}, \mathscr{G}_{U}\right)$ is not an isomorphism, see below (1.6).

\section{Coherent sheaves}

A sheaf $\mathscr{F}$ of $\mathscr{O}_{X}$-modules is locally coherent, if for every $x / U$ the sheaf $\mathscr{F}_{U}$ is a coherent sheaf of $\mathscr{O}_{U_{\text {ét }}}$-modules. (This terminology is inspired by 18 , Tag 06WJ.) It is cartesian, if all compatibility morphisms $\alpha^{*}: f^{*} \mathscr{F}_{U} \rightarrow$ $\mathscr{F}_{V}$ are isomorphisms of sheaves of $\mathscr{O}_{V_{\text {ét }}}$-modules. A sheaf which is both locally coherent and cartesian is coherent.

For example, a groupoid presentation $X_{1} \rightrightarrows X_{0}$ of $X$, and a coherent sheaf $\mathscr{F}_{0}$ on $X_{0}$, together with an isomorphism $s^{*} \mathscr{F}_{0} \rightarrow t^{*} \mathscr{F}_{0}$, satisfying the usual cocycle condition on $X_{2}=X_{1} \times X_{0} X_{1}$, give rise to a coherent sheaf on $X$.

The sheaf of sections of a vector bundle over $X$ is coherent. In fact, the notion of vector bundle and locally free coherent sheaf are equivalent, and we will use them interchangeable, even though the two categories are anti-equivalent. The cokernel of a homomorphism of vector bundles is coherent. In fact, every cokernel of a homomorphism of coherent sheaves is coherent.

If the cokernel of a homomorphism of vector bundles is locally free, we 
call the homomorphism a strict homomorphism of vector bundles. For a strict homomorphism of vector bundles, the image and the kernel, as well as the cokernel are locally free.

A strict monomorphism of vector bundles is a strict homomorphism whose kernel is zero. A homomorphism of vector bundles is a strict monomorphism/an epimorphism, if and only if over every geometric point of $X$, the induced linear map is injective/surjective. A homomorphism of vector bundles, which is a monomorphism of sheaves, is a strict monomorphism of bundles.

Let $\varphi: E \rightarrow F$ be a homomorphism of vector bundles over the algebraic stack $X$. The flattening stratification $X^{\prime} \rightarrow X$ of $\operatorname{cok} \varphi$ serves also as strictening stratification for $\varphi$. This means that an object of $X(S)$ lifts to $X^{\prime}(S)$, if and only if $\varphi_{S}$ is strict.

In general, the kernel (in the category of big sheaves of $\mathscr{O}_{X}$-modules) of a homomorphism of vector bundles is locally coherent, but not coherent.

By [18, Tag 06WK], a sheaf of $\mathscr{O}_{X}$-modules $\mathscr{F}$ is coherent if and only if there exists a smooth covering $X_{i} \rightarrow X$ of $X$ by finite type affine schemes $X_{i}$, such that for every $i$, the restriction $\mathscr{F}_{i}$ of $\mathscr{F}$ to the big étale site of $X_{i}$ is isomorphic to the cokernel of a homomorphism of vector bundles.

Proposition 1.1. Suppose that $\mathscr{F}$ is a coherent sheaf on the algebraic stack $X$. Then

(i) for every $x / U$, we have $\left(\mathscr{F}^{\vee}\right)_{U}=\left(\mathscr{F}_{U}\right)^{\vee}$,

(ii) $\mathscr{F}^{\vee}$ is locally coherent,

(iii) $\mathscr{F}^{\vee}$ is represented by a an algebraic stack, which is of finite type and affine over $X$, namely $\operatorname{Spec}_{X} \operatorname{Sym}_{\mathscr{O}_{X}} \mathscr{F}$,

(iv) the canonical homomorphism $\mathscr{F} \rightarrow \mathscr{F}^{\vee \vee}$ is an isomorphism of sheaves of $\mathbb{O}_{X}$-modules,

(v) if $\mathscr{F}$ is locally free coherent, then $\mathscr{F}^{\vee}$ is a vector bundle.

Moreover, the functor $\mathscr{F} \mapsto \mathscr{F}^{\vee}$ is a fully faithful functor from the category of coherent sheaves to the category of locally coherent sheaves of $\mathbb{O}_{X}$-modules. It maps right exact sequences of coherent sheaves to left exact sequences of locally coherent sheaves.

Proof. The first claim follows directly from the definitions, the main fact being the adjunction

$$
\operatorname{Hom}_{\mathscr{C}_{U}}\left(\mathscr{F}_{U}, f_{*} \mathscr{O}_{V}\right)=\operatorname{Hom}_{\mathscr{C}_{V}}\left(f^{*} \mathscr{F}_{U}, \mathscr{O}_{V}\right)=\operatorname{Hom}_{\mathscr{C}_{V}}\left(\mathscr{F}_{V}, \mathscr{O}_{V}\right)
$$

for every morphism of $X$-schemes $f: V \rightarrow U$.

The second claim follows from the first (see also [18, Tag 06WM]).

For the third claim, see [15, (14.2.6)].

For the fourth claim, consider the sheaf of $\mathbb{G}_{m}$-equivariant $X$-morphisms from $\operatorname{Spec}_{X} \operatorname{Sym}_{\mathscr{C}_{X}} \mathscr{F}$ to $\mathbb{A}_{X}^{1}$, denoted by $\underline{\operatorname{Hom}}_{\mathbb{G}_{m}}\left(\mathscr{F}^{\vee}, \mathbb{A}^{1}\right)$. It is equal to the sheaf of homomorphisms of graded $\mathscr{O}_{X}$-algebras from $\mathscr{O}_{X}[t]$ to $\operatorname{Sym}_{\mathscr{O}_{X}} \mathscr{F}$, hence equal to $\mathscr{F}$. But sections of $\mathscr{F}$ give rise to $\mathscr{O}_{X}$-linear homomorphisms $\mathscr{F}^{\vee} \rightarrow \mathscr{O}_{X}$, not just $\mathbb{G}_{m}$-equivariant ones. Hence

$$
\mathscr{F}=\underline{\operatorname{Hom}}_{\mathbb{G}_{m}}\left(\mathscr{F}^{\vee}, \mathbb{A}^{1}\right)=\mathscr{H} \operatorname{mom}_{\mathscr{O}_{X}}\left(\mathscr{F}^{\vee}, \mathscr{O}_{X}\right)=\mathscr{F}^{\vee} \vee
$$


The fifth claim is clear.

The 'moreover' follows from the fact that we can reconstruct $\mathscr{F}$ from $\mathscr{F}^{\vee}=\operatorname{Spec}_{X} \operatorname{Sym}_{\mathscr{C}_{X}} \mathscr{F}$, as the degree one part of the the graded sheaf of $\mathscr{O}_{X}$-modules $\pi_{*}\left(\mathscr{O}_{\mathscr{F}}\right)$, where $\pi: \mathscr{F} \rightarrow X$ is the projection morphism.

\section{Representable sheaves of modules}

If $\varphi: E \rightarrow F$ is a homomorphism of vector bundles over $X$, then $\operatorname{ker} \varphi$, constructed in the category of big sheaves, is a representable sheaf of $\mathscr{O}_{X}$-modules. In fact, $\operatorname{ker} \varphi$ is equal to the fibered product of stacks

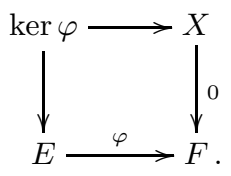

Sheaves such as $\operatorname{ker} \varphi$ belong to a class of $\mathfrak{O}_{X}$-modules dual to coherent sheaves.

Proposition 1.2. Let $\mathscr{F}_{\text {be }}$ a sheaf of $\mathscr{O}_{X}$-modules. The following are equivalent:

(i) there exists a coherent sheaf $\mathcal{N}$, such that $\mathscr{F}$ is isomorphic to $\mathcal{N}^{\vee}$,

(ii) there exists a smooth cover $X_{i} \rightarrow X$ of $X$ by finite type affine schemes $X_{i}$, such that, for every $i$, the restriction $\mathscr{F}_{i}$ of $\mathscr{F}_{\text {to }}$ the big étale site of $X_{i}$ is isomorphic to the kernel of a homomorphism of vector bundles over $X_{i}$.

Proof. The fact that (i) implies (ii), follows from the results proved above. So let us indicate how to prove that (ii) implies (i).

Let us first assume that $\mathscr{F}$ is isomorphic to the kernel of a homomorphism of vector bundles $E_{0} \rightarrow E_{1}$. One checks that $\mathscr{F}$ is then represented by $\operatorname{Spec}_{X} \operatorname{Sym}_{\mathscr{O}_{X}} \operatorname{cok}\left(E_{1}^{\vee} \rightarrow E_{0}^{\vee}\right)$. Thus $\mathscr{F}$ is isomorphic to the dual of the coherent sheaf $\operatorname{cok}\left(E_{1}^{\vee} \rightarrow E_{0}^{\vee}\right)$.

Now suppose that $\mathscr{F}$ is locally isomorphic to the kernel of a homomorphism of vector bundles. It suffices to prove that $\mathscr{F}^{\vee}$ is coherent, and that $\mathscr{F} \rightarrow \mathscr{F}^{\vee \vee}$ is an isomorphism. Both claims can be checked locally, and are true for duals of coherent sheaves.

Definition 1.3. We call a sheaf of $\mathscr{O}_{X}$-modules locally coherent representable, if any of the two equivalent conditions of Proposition 1.2 is satisfied. The terminology is justified by Proposition 1.4 below.

In other words, the category of locally coherent representable sheaves over $X$ is the essential image of the fully faithful functor mentioned in Proposition 1.1. We therefore have an equivalence of categories

$$
\begin{aligned}
\text { (coh. sheaves over } X) & \longrightarrow \text { (loc. coh. repr. sheaves over } X) \\
\mathscr{F} & \longmapsto \mathscr{F}^{\vee} .
\end{aligned}
$$

The following proposition summarizes facts about locally coherent representable sheaves, which all follow easily from facts mentioned above. 
Proposition 1.4. Let $\mathscr{F}$ be a locally coherent representable sheaf over the algebraic stack $X$. Then

(i) the sheaf $\mathscr{F}$ is locally coherent,

(ii) the sheaf $\mathscr{F}^{\vee}$ is coherent,

(iii) the canonical homomorphism $\mathscr{F} \rightarrow \mathscr{F} \vee \vee$ is an isomorphism of sheaves of $\mathfrak{O}_{X}$-modules,

(iv) the sheaf $\mathscr{F}$ is representable by an algebraic stack $F \rightarrow X$, which is of finite type and affine over $X$,

(v) in fact, $\mathscr{F}=\operatorname{Spec}_{X} \operatorname{Sym}_{\mathscr{O}_{X}} \mathscr{F} \vee$.

Moreover, the functor $\mathscr{F} \mapsto \mathscr{F} \vee$ is an essential inverse to the functor (5). It maps left exact sequences of locally coherent representable sheaves to right exact sequences of coherent sheaves.

Proposition 1.5. Let $\mathscr{F}$ be a locally coherent representable sheaf over the finite type algebraic stack $X$. There is a unique stratification $X^{\prime} \rightarrow X$, with the property that an $X$-scheme $S$ factors through $X^{\prime}$, if and only if $\left.\mathscr{F}\right|_{S}$ is a vector bundle. More precisely, $X^{\prime}=\coprod_{n>0} X_{n}$, and $X_{n} \rightarrow X$ is a locally closed immersion of algebraic stacks, with the property that $S \rightarrow X$ factors through $X_{n}$ if and only if $\mathscr{F}_{S_{S}}$ is a vector bundle of rank $n$.

Proof. The sought after stratification is the flattening stratification of the coherent sheaf $\mathscr{F}^{\vee}$.

Example 1.6. Consider $X=\mathbb{A}^{1}$, with coordinate $t$, and let $\mathscr{C}$ be the cokernel of the homomorphism of vector bundles $t: \mathbb{A}_{X}^{1} \rightarrow \mathbb{A}_{X}^{1}$. It is the skyscraper sheaf of the origin, considered as a coherent sheaf on $X$, and extended to a big sheaf over $X$ in the usual way. The sheaf $\mathscr{C}$ is an example of a coherent sheaf which is not representable.

Let $\mathscr{K}$ be the kernel of $t: \mathbb{A}_{X}^{1} \rightarrow \mathbb{A}_{X}^{1}$. This is locally coherent representable, but not cartesian, hence not coherent.

Note that $\mathscr{C}^{\vee}=\mathscr{K}$. This shows that $\mathscr{F}^{\vee}$ may not be coherent, even if $\mathscr{F}$ is.

Note also, that $\mathscr{K}^{\vee}=\mathscr{C}$, which shows that $\mathscr{F}^{\vee}$ may not be representable, even if $\mathscr{F}$ is.

Finally, note that $\left(\mathscr{K}_{X}\right)^{\vee}=0^{\vee}=0$, but $\left(\mathscr{K}^{\vee}\right)_{X}=\mathscr{C}_{X}$ is the structure sheaf of the origin in $X$, considered as a skyscraper sheaf on $X_{\text {ét }}$, which is not zero. This gives an example where $\left(\mathscr{F}^{\vee}\right)_{U} \neq\left(\mathscr{F}_{U}\right)^{\vee}$.

Remark 1.7. Of course the category of coherent sheaves on an algebraic stack $X$ has kernels and internal homs, but they do not agree with those in the category of big sheaves, which we considered above. It is therefore important to specify the context, when dealing with kernels or duals in the category of coherent sheaves. Unless specified otherwise, we will always consider sheaves of $\mathscr{O}_{X}$-modules as big sheaves. 


\subsection{Linear algebraic stacks}

We will review the definition of linear algebraic stacks, and some basic constructions. For definitions and basic properties of fibered categories we refer the reader to [8, Exposé VI]. The material here is presumably known, but we could not find a suitable reference.

Suppose $\mathfrak{X} \rightarrow \mathscr{I}$ is a category over $\mathscr{S}$. We write $\mathfrak{X}(S)$ for the fiber of $\mathfrak{X}$ over the object $S$ of $\mathscr{S}$. If $f: S^{\prime} \rightarrow S$ is a morphism in $\mathscr{S}$, and $x^{\prime} \in \mathfrak{X}\left(S^{\prime}\right)$ and $x \in \mathfrak{X}(S)$ are $\mathfrak{X}$-objects lying over $S^{\prime}$ and $S$, respectively, we write $\operatorname{Hom}_{f}\left(x^{\prime}, x\right)$ for the set of morphisms from $x^{\prime}$ to $x$ in $\mathfrak{X}$, lying over $f$. For $S^{\prime}=S$ and $f=\operatorname{id}_{S}$, we write $\operatorname{Hom}_{S}\left(x^{\prime}, x\right)$.

Recall that a morphism $\alpha: x^{\prime} \rightarrow x$ lying over $f: S^{\prime} \rightarrow S$ is cartesian, if for every object $x^{\prime \prime}$ of $\mathfrak{X}(S)$, composition with $\alpha$ induces a bijection $\operatorname{Hom}_{S}\left(x^{\prime \prime}, x^{\prime}\right) \stackrel{\simeq}{\longrightarrow} \operatorname{Hom}_{f}\left(x^{\prime \prime}, x\right)$. Recall further that $\mathfrak{X} \rightarrow \mathscr{S}$ is a fibered category, if every composition of cartesian morphisms is cartesian, and if for every $f: S^{\prime} \rightarrow S$ in $\mathscr{S}$, and every $x$ over $S$, there exists a cartesian morphism over $f$ with target $x$. A cartesian functor between categories over $\mathscr{S}$ is one that preserves cartesian morphisms.

If $\mathfrak{X}$ is a fibered category over $\mathscr{I}$, the subcategory of $\mathfrak{X}$, consisting of the same objects and all cartesian morphisms is a category fibered in groupoids over $\mathscr{S}$. We denote it by $\mathfrak{X}_{\mathrm{cfg}}$, and call it the underlying category fibered in groupoids.

Definition 1.8. A category $\mathfrak{X}$ over $\mathscr{S}$ is an $\mathscr{O}$-linear category over $\mathscr{S}$, if for every $f: S^{\prime} \rightarrow S$ in $\mathscr{S}$ and all $x^{\prime} \in \mathfrak{X}\left(S^{\prime}\right), x \in \mathfrak{X}(S)$, the set $\operatorname{Hom}_{f}\left(x^{\prime}, x\right)$ is endowed with the structure of an $\mathscr{O}\left(S^{\prime}\right)$-module, in such a way that for every pair of morphisms $g: S^{\prime \prime} \rightarrow S^{\prime}, f: S^{\prime} \rightarrow S$, and every triple of objects $x^{\prime \prime} \in \mathfrak{X}\left(S^{\prime \prime}\right), x^{\prime} \in \mathfrak{X}\left(S^{\prime}\right), x \in \mathfrak{X}(S)$, the composition

$$
\operatorname{Hom}_{f}\left(x^{\prime}, x\right) \times \operatorname{Hom}_{g}\left(x^{\prime \prime}, x^{\prime}\right) \longrightarrow \operatorname{Hom}_{f \circ g}\left(x^{\prime \prime}, x\right)
$$

is $\mathbb{O}\left(S^{\prime}\right)$-bilinear.

An $\mathscr{O}$-linear functor $F: \mathfrak{X} \rightarrow \mathfrak{Y}$ between $\mathscr{O}$-linear categories is a functor of categories over $\mathscr{S}$, such that for every $f: S^{\prime} \rightarrow S$, and all $x^{\prime} \in \mathfrak{X}\left(S^{\prime}\right), x \in \mathfrak{X}(S)$ the $\operatorname{map} \operatorname{Hom}_{f}\left(x^{\prime}, x\right) \rightarrow \operatorname{Hom}_{f}\left(F\left(x^{\prime}\right), F(x)\right)$ is $\mathscr{O}\left(S^{\prime}\right)$-linear.

Assume given an $\mathscr{O}$-linear fibered category $\mathfrak{X}$ over $\mathscr{S}$. Pullback in $\mathfrak{X}$ is $\mathscr{O}$-linear, i.e., if $f: S^{\prime} \rightarrow S$ is a morphism in $\mathscr{S}$, and $x, y \in \mathfrak{X}(S)$ are objects with pullbacks $x^{\prime}, y^{\prime} \in \mathfrak{X}\left(S^{\prime}\right)$, the pullback map $f^{*}: \operatorname{Hom}_{S}(x, y) \rightarrow$ $\operatorname{Hom}_{S^{\prime}}\left(x^{\prime}, y^{\prime}\right)$ is $\mathscr{O}(S)$-linear.

So if we fix objects $x, y \in \mathfrak{X}(S)$, the presheaf $\underline{\operatorname{Hom}}_{S}(x, y)$ over the usual small étale site of $S$, defined by $\underline{\operatorname{Hom}}_{S}(x, y)(T)=\operatorname{Hom}_{T}\left(\left.x\right|_{T},\left.y\right|_{T}\right)$, for every étale $T \rightarrow S$, is a presheaf of $\mathscr{O}_{S_{\text {ét }}}$-modules. Moreover, for any morphism $f: S^{\prime} \rightarrow S$ in $\mathscr{S}$, we have a natural homomorphism of presheaves of $\mathscr{O}_{S}$-modules $\underline{\operatorname{Hom}}_{S}(x, y) \rightarrow f_{*} \underline{\operatorname{Hom}}_{S^{\prime}}\left(x^{\prime}, y^{\prime}\right)$. Put together, the small presheaves $\underline{\operatorname{Hom}}_{T}(x, y)$, as $T \rightarrow S$ varies over the big étale site of the scheme $S$, form a big presheaf, which we denote by $\underline{\operatorname{Hom}}(x, y)$.

Definition 1.9. A linear algebraic stack is an $\mathscr{O}$-linear fibered category $\mathfrak{X}$ over $\mathscr{S}$, such that 
(i) for every object $S \in \mathscr{S}$, and every pair $x, y \in \mathfrak{X}(S)$, the presheaf $\underline{\operatorname{Hom}}(x, y)$ on the big étale site of the scheme $S$ is a locally coherent representable sheaf of $\mathbb{O}_{S}$-modules,

(ii) the underlying category fibered in groupoids $\mathfrak{X}_{\mathrm{cfg}} \rightarrow \mathscr{S}$ is an algebraic stack over $R$ (locally of finite type).

A morphism of linear algebraic stacks is an $\mathbb{O}$-linear cartesian functor over $\mathscr{S}$

Remark 1.10. If $\mathfrak{X}$ is a linear algebraic stack, with underling algebraic stack $X=\mathfrak{X}_{\text {cfg }}$, there exists a locally coherent representable sheaf $\mathscr{H}$ over $X \times X$, which represents the sheaf over $X \times X$, whose set of sections over the pair $x, y \in X(S)$ is the $\mathscr{O}(S)$-module $\operatorname{Hom}_{S}(x, y)$. The sheaf $\mathscr{H}$ is the universal sheaf of homomorphisms. The subsheaf $\mathscr{I} \subset \mathscr{H}$ representing isomorphisms is naturally identified with $X$, and the projection to $X \times X$ with the diagonal.

Pulling back $\mathscr{H}$ via the diagonal to $X$, we obtain the universal sheaf of endomorphisms $\mathscr{E} \rightarrow X$, which represents the sheaf whose set of sections over $x \in X(S)$ is the $\mathscr{O}(S)$-algebra $\operatorname{End}_{S}(x)$. Let us emphasize that $\mathscr{E} \rightarrow X$ is a representable morphism of algebraic stacks, which is at the same time a sheaf of algebras, and a locally coherent representable sheaf of $\mathscr{O}_{X}-$ modules.

The linear algebraic stack $\mathfrak{X}$ can be reconstructed from its underlying algebraic stack $X$, and the representable sheaf of $\mathscr{O}_{X \times X}$-algebras $\mathscr{H}$. We leave it to the reader to write down axioms for the pair $(X, \mathscr{H})$, which assure that $(X, \mathscr{H})$ comes from a linear algebraic stack.

\section{Examples}

Example 1.11. Let $X$ be a projective $R$-scheme. The linear stack $\mathfrak{C o h}_{X}$ has as objects lying over the $R$-scheme $S$, the coherent sheaves on $X \times$ $S$, which are flat over $S$. For a morphism of $R$-schemes $f: S^{\prime} \rightarrow$ $S$, and $\mathscr{F}^{\prime} \in \mathfrak{C o h}_{X}\left(S^{\prime}\right)$, and $\mathscr{F} \in \mathfrak{C o h}_{X}(S)$, we set $\operatorname{Hom}_{f}\left(\mathscr{F}^{\prime}, \mathscr{F}\right)=$ $\operatorname{Hom}_{\mathscr{C}_{X \times S^{\prime}}}\left(\mathscr{F}^{\prime}, f^{*} \mathscr{F}\right)$. A morphism $\mathscr{F}^{\prime} \rightarrow \mathscr{F}$ in $\mathfrak{C o h}_{X}$ over $f$ in $\mathscr{S}$ is cartesian, if it induces an isomorphism $\mathscr{F}^{\prime} \cong f^{*} \mathscr{F}$.

The linear stack $\mathfrak{C o h}_{X}$ is algebraic.

To see this, suppose $\mathscr{F}$ and $\mathscr{G}$ are coherent sheaves on $X \times S$, flat over $S$. The fact that $\underline{\operatorname{Hom}}(\mathscr{F}, \mathscr{G})$ is a locally coherent representable sheaf of $\mathscr{O}_{X}$-modules, follows from the fact that there exists a coherent sheaf $\mathcal{N}$ on the big étale site of $S$, such that $\underline{\operatorname{Hom}}(\mathscr{F}, \mathscr{G})=\mathcal{N}^{\vee}$ (see [9], EGA III 7.7.8, 7.7.9]). In fact, for a morphism of schemes $T \rightarrow S$, we have $\underline{\operatorname{Hom}}_{T}(\mathscr{F}, \mathscr{G})=\pi_{T *} \mathscr{H} \operatorname{lom}\left(\mathscr{F}_{X \times T}, \mathscr{G}_{X \times T}\right)$. The fact that pushforward does not commute with arbitrary pullbacks means that $\underline{\operatorname{Hom}}(\mathscr{F}, \mathscr{G})$ is not in general cartesian, and hence not in general coherent. On the other hand, by [ibid.], we have $\pi_{T *} \mathscr{H} \operatorname{lom}\left(\mathscr{F}_{X \times T}, \mathscr{G}_{X \times T}\right)=\left(\mathcal{N}_{T}\right)^{\vee}$, which proves that, indeed, $\underline{\operatorname{Hom}}(\mathscr{F}, \mathscr{G})=\mathcal{N}^{\vee}$.

The fact that $\left(\mathfrak{C o h}_{X}\right)_{\text {cfg }}$ is algebraic and locally of finite type is proved in [15, 4.6.2.1.]. 
Example 1.12. As a special case of the previous example, consider the case $X=\operatorname{Spec} R$. Then the linear algebraic stack $\mathfrak{C o h}_{\operatorname{Spec} R}$ is the linear stack of vector bundles, notation $\mathfrak{V e c t}$. The underlying algebraic stack $\mathfrak{V e c t}_{\text {cfg }}$ is the disjoint union $\coprod_{n \geq 0} B G L_{n}$. The sheaf $\mathscr{H}$ over

$$
\coprod_{n \geq 0} B G L_{n} \times \coprod_{n \geq 0} B G L_{n}=\coprod_{n, m \geq 0} B\left(G L_{n} \times G L_{m}\right)
$$

is given by the natural representation $M(m \times n)$ of $G L_{n} \times G L_{m}$ over the component $B\left(G L_{n} \times G L_{m}\right)$.

Example 1.13. A generalization of $\mathfrak{V e c t}$ in a different direction is given by quiver representations.

Let $Q$ be a quiver. The stack of representations of $Q$, notation $\mathfrak{R e p}_{Q}$, has as $\mathfrak{R e p}_{Q}(S)$ the set of diagrams $(\mathscr{F})$ in the shape of $Q$ of locally free finite rank $\mathscr{O}_{S}$-modules. For a morphism $f: S^{\prime} \rightarrow S$ of $R$-schemes we have that $\operatorname{Hom}_{f}\left(\mathscr{F}^{\prime}, \mathscr{F}\right)$ is the $\mathscr{O}\left(S^{\prime}\right)$-module of homomorphisms $\mathscr{F}^{\prime} \rightarrow f^{*} \mathscr{F}$ of diagrams of locally free $\mathscr{O}_{S^{\prime}}$-modules.

Example 1.14. As a toy example, let $A$ be a smooth $R$-algebra scheme of finite type, with smooth group scheme of units $A^{\times}$, also of finite type, such that the underlying $R$-module is locally free. Then we define the linear stack of $A^{\times}$-torsors to have as objects over the $R$-scheme $S$ the right $A^{\times}$-torsors over $S$, and for $f: S^{\prime} \rightarrow S$ and $A^{\times}$-torsors $P^{\prime}$ over $S^{\prime}$ and $P$ over $S$, we set $\operatorname{Hom}_{f}\left(P^{\prime}, P\right)=\operatorname{Hom}_{S^{\prime}}\left(P^{\prime}, f^{*} P\right)=P^{\prime} \times_{A} \times A \times_{A \times} f^{*} P$. In this example, the underlying algebraic stack is $B A^{\times}$and we have $\mathscr{H}=$ $A^{\times} \backslash A / A^{\times}$.

The case $A=0$ is not excluded. The associated linear stack is id : $\mathscr{S} \rightarrow \mathscr{I} . \operatorname{All}_{\operatorname{Hom}_{f}}(x, y)$ are singletons, endowed with their unique module structure. This stack is represented by $\operatorname{Spec} R$. It can also be thought of as the stack of zero-dimensional vector bundles.

\section{Substacks}

Let $\mathfrak{X}$ be a linear algebraic stack with underlying algebraic stack $X=\mathfrak{X}_{\text {cfg }}$. If $Y \subset X$ is a locally closed algebraic substack, there is a canonical linear algebraic stack $\mathfrak{Y}$, with underlying algebraic stack $\mathfrak{Y}_{\text {cfg }}=Y$. In fact, we can define $\mathfrak{Y}$ to be the full subcategory of $\mathfrak{X}$ consisting of objects which are in $X$.

In this situation, we call $\mathfrak{Y} \rightarrow \mathfrak{X}$ a locally closed linear substack of $\mathfrak{X}$.

\section{Fibered products}

Let $F: \mathfrak{X} \rightarrow \mathfrak{Z}$ and $G: \mathfrak{Y} \rightarrow \mathfrak{Z}$ be cartesian morphisms of $\mathfrak{O}$-linear fibered categories. We define a new $\mathscr{O}$-linear fibered category $\mathfrak{W}$ as follows: objects of $\mathfrak{W}$ over the object $T$ of $\mathscr{S}$ are triples $(x, \alpha, y)$, where $x$ is an $\mathfrak{X}$ object over $T, y$ is a $\mathfrak{Y}$-object over $T$, and $\alpha$ is an isomorphism $\alpha: F(x) \rightarrow$ $G(y)$, over $T$. A morphism from $\left(x^{\prime}, \alpha^{\prime}, y^{\prime}\right)$ to $(x, \alpha, y)$ over $T^{\prime} \rightarrow T$ is a 
pair of morphisms $f: x^{\prime} \rightarrow x$ over $T^{\prime} \rightarrow T$ and $g: y^{\prime} \rightarrow y$ over $T^{\prime} \rightarrow T$, such that $\alpha \circ F(f)=G(g) \circ \alpha^{\prime}$.

In other words, we can write the set of morphisms from $\left(x^{\prime}, \alpha^{\prime}, y^{\prime}\right)$ to $(x, \alpha, y)$ over $\varphi: T^{\prime} \rightarrow T$ as the fibered product

$$
\operatorname{Hom}_{\varphi}\left(x^{\prime}, x\right) \times_{\operatorname{Hom}_{\varphi}\left(F\left(x^{\prime}\right), G(y)\right)} \operatorname{Hom}_{\varphi}\left(y^{\prime}, y\right),
$$

and as each of the sets in this fibered product is an $\mathscr{O}\left(T^{\prime}\right)$-module, and the maps are linear, this fibered product is also an $\mathbb{O}\left(T^{\prime}\right)$-module. We leave it to the reader to verify that composition is bilinear.

Let us verify that $\mathfrak{W}$ is a fibered category. Suppose that $(x, \alpha, y)$ is a triple over $T$, and $\varphi: T^{\prime} \rightarrow T$ a morphism in $\mathscr{S}$. We construct a triple $\left(x^{\prime}, \alpha^{\prime}, y^{\prime}\right)$ over $T^{\prime}$ by taking as $x^{\prime}$ a pullback of $x$ via $\varphi$, and for $y^{\prime}$ a pullback of $y$ via $\varphi$. Then, as $G$ is cartesian, $G\left(y^{\prime}\right)$ is a pullback of $G(y)$ via $\varphi$. Hence there exists a unique morphism $\alpha^{\prime}: F\left(x^{\prime}\right) \rightarrow$ $G\left(y^{\prime}\right)$ covering $T^{\prime}$, such that $\alpha \circ F\left(x^{\prime} \rightarrow x\right)=G\left(y^{\prime} \rightarrow y\right) \circ \alpha^{\prime}$. Then $\alpha^{\prime}$ is cartesian, because cartesian morphisms satisfy the necessary two out of three property. Then $\alpha^{\prime}$ is invertible, because cartesian morphisms covering an identity are invertible. The triple $\left(x^{\prime}, \alpha^{\prime}, y^{\prime}\right)$ comes with a given morphism to $(x, \alpha, y)$ which covers $\varphi$. It is easily verified that this morphism is cartesian.

Therefore, $\mathfrak{W}$ is an $\mathfrak{O}$-linear fibered category. By construction, the two projections $\mathfrak{W} \rightarrow \mathfrak{X}$ and $\mathfrak{W} \rightarrow \mathfrak{Y}$ are cartesian. We call $\mathfrak{W}$ the fibered product of $\mathfrak{X}$ and $\mathfrak{Y}$ over $\mathfrak{Z}$.

Suppose $\mathfrak{X}, \mathfrak{Y}$ and $\mathfrak{Z}$ are algebraic, with underlying algebraic stacks $X, Y$ and $Z$, respectively. For triples $\left(x^{\prime}, \alpha^{\prime}, y^{\prime}\right)$ and $(x, \alpha, y)$ over $S$, the presheaf $\underline{\text { Hom }}\left(\left(x^{\prime}, \alpha^{\prime}, y^{\prime}\right),(x, \alpha, y)\right)$ is equal to the fibered product

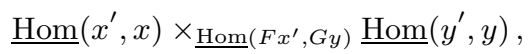

and is therefore a locally coherent representable sheaf of $\mathfrak{O}_{S}$-modules. We see that $\mathfrak{W}$ is again a linear algebraic stack. Moreover, the underlying algebraic stack of $W$ is the fibered product $X \times{ }_{Z} Y$.

\section{Lack of locality}

Remark 1.15. Suppose $\mathfrak{X}$ and $\mathfrak{Y}$ are linear algebraic stacks, with underlying algebraic stacks $X$ and $Y$. We can construct a disjoint union linear algebraic stack $\mathfrak{X} \amalg \mathfrak{Y}$ whose underlying algebraic stack is $X \amalg Y$, by declaring all homomorphisms between objects of $\mathfrak{X}$ and objects of $\mathfrak{Y}$ to be zero. This concept of disjoint union is not useful for our purposes. For the linear algebraic stacks we are interested in, the underlying algebraic stack often decomposes into a disjoint union, even though the linear algebraic stack does not. An example is given by the linear stack of vector bundles $\mathfrak{V} \mathfrak{c} \mathfrak{c t}$, Example 1.12

Thus linear algebraic stacks exhibit less local behaviour than algebraic stacks, and are therefore less geometrical. This is one of the reasons we prefer to work with algebroids, rather than linear algebraic stacks. 


\section{Special linear stacks}

For a linear algebraic stack $\mathfrak{M}$, every fiber category $\mathfrak{M}(S)$ is an $R$-linear category. By putting special requirements on these linear categories, we get stronger notions of linear algebraic stack.

For a linear algebraic stack $\mathfrak{M}$, we denote the universal sheaf of endomorphisms by $\mathfrak{A} \rightarrow \mathfrak{M}$.

Definition 1.16. A linear algebraic stack $\mathfrak{M}$ has a zero object, if the $R$-linear category $\mathfrak{M}(R)$ admits a zero object.

If $\mathfrak{M}$ admits a zero object, then for every $R$-scheme $S$, the $R$-linear category $\mathfrak{M}(S)$ admits a zero object, namely the pullback of the zero object in $\mathfrak{M}(\operatorname{Spec} R)$ via the unique morphism $S \rightarrow \operatorname{Spec} R$.

A zero object for $\mathfrak{M}$ defines a section $\operatorname{Spec} R \stackrel{0}{\longrightarrow} \mathfrak{M}$, which is an isomorphism onto the closed substack of $\mathfrak{M}$ defined by the condition $1=0$ inside $\mathfrak{A}$.

If $\mathfrak{M}$ admits a zero object, we denote the complement of the zero object in $\mathfrak{M}$ by $\mathfrak{M}_{*}$. It is a linear open substack of $\mathfrak{M}$.

Definition 1.17. The linear algebraic stack $\mathfrak{M}$ admits direct sums, if for ever $R$-scheme $S$, the $R$-linear category $\mathfrak{M}(S)$ admits all (finite) direct sums.

The pullback functor $\mathfrak{M}(S) \rightarrow \mathfrak{M}\left(S^{\prime}\right)$, for a morphism of $R$-schemes $S^{\prime} \rightarrow S$ commutes with direct sums. Hence, if $\mathfrak{M}$ admits direct sums, there is a canonical morphism of linear stacks

$$
\begin{aligned}
\mathfrak{M} \times \mathfrak{M} & \longrightarrow \mathfrak{M} \\
(x, y) & \longmapsto x \oplus y .
\end{aligned}
$$

See also Remark 1.63 


\subsection{Finite type algebras}

Definition 1.18. Let $X$ be an algebraic stack. By an algebra over $X$, we mean a sheaf of $\mathscr{O}_{X}$-algebras over $X$. If the algebra $A$ over the algebraic stack $X$ is an algebraic stack itself, i.e., if the structure morphism $A \rightarrow X$ is a representable morphism of stacks, then we say that $A$ is representable. If $A$ is represented by a finite type affine stack of the form $\operatorname{Spec}_{X} \operatorname{Sym}_{\mathscr{O}_{X}} \mathscr{F}$, for a coherent sheaf $\mathscr{F}$ over $X$, we call $A$ a finite type algebra over $X$.

For an automorphism $\varphi$ of an algebra $A$, we denote the subalgebra of fixed sections by $A^{\varphi}$. For a section $a$ of $A$ we denote by $A^{a}$ the subalgebra of sections commuting with $a$.

So the sheaf of $\mathscr{O}_{X}$-modules underlying a finite type algebra is locally coherent representable.

If $\mathfrak{X}$ is a linear algebraic stack with underlying algebraic stack $X$, then the universal sheaf of endomorphisms $\mathscr{E} \rightarrow X$ is a finite type algebra.

Note that finite type algebras need not have a coherent underlying sheaf of $\mathscr{O}_{X}$-modules. For example, let $X=\mathbb{A}^{1}$, with coordinate $t$, and let $A \rightarrow X$ be the centralizer in $\left(M_{2 \times 2}\right)_{X}$ of the matrix $\left(\begin{array}{ll}t & 0 \\ 0 & 0\end{array}\right)$. In this case the big sheaf underlying $A$ is not cartesian.

Remark 1.19. Dually, a finite type algebra $A$ over $X$ corresponds to a coherent sheaf $M$ over $X$, which is endowed with a coalgebra structure $(\Delta, \varepsilon)$, where $\Delta: M \rightarrow M \otimes_{\mathscr{O}_{X}} M$ is an associative comultiplication with counit $\varepsilon: M \rightarrow \mathscr{O}_{X}$. For any $X$-scheme $U$, we have $A_{U}=M_{U}^{\vee}$.

\section{Inertia representation}

Whenever $A \rightarrow X$ is an algebra over the algebraic stack $X$, we have a tautological morphism of sheaves of groups over $X$

$$
I_{X} \longrightarrow \underline{\operatorname{Aut}}(A) \text {. }
$$

Here $I_{X}$ is the inertia stack of $X$, i.e., the stack of pairs $(x, \varphi)$, where $x$ is an object of $X$, and $\varphi$ an automorphism of $x$, and $\underline{\operatorname{Aut}}(A)$ is the sheaf of automorphisms of the sheaf of algebras $A$ over $X$. To construct (6), consider the stack of sheaves of algebras $\mathfrak{A} \mathfrak{g}$ over $\mathscr{S}$, which has as objects over the scheme $S$, the sheaves of $\mathscr{O}_{S}$-algebras on the usual (small) étale site of $S$. A morphism from the sheaf of $\mathscr{O}_{S^{\prime}}$-algebras $A^{\prime}$ over $S^{\prime}$, covering the morphism of schemes $f: S^{\prime} \rightarrow S$, to the sheaf of $\mathscr{O}_{S^{-}}$-algebras $A$ over $S$, is, by definition, an isomorphism of sheaves of $\mathscr{O}_{S^{\prime}}$-algebras $A^{\prime} \rightarrow f^{*} A$. The sheaf of algebras $A \rightarrow X$ gives rise to a morphism of $\mathscr{S}$-stacks $a: X \rightarrow \mathfrak{A} \mathfrak{l g}$. We get an induced morphism on inertia stacks $I_{X} \rightarrow I_{\mathfrak{A} \mathfrak{g}}$, and notice that $a^{*} I_{\mathfrak{A} \mathfrak{g}}=\underline{\operatorname{Aut}}(A)$.

With this definition, an automorphism $\varphi$ of the object $x$ of the stack $X$ is mapped to the inverse of the restriction morphism $\varphi^{*}: A(x) \rightarrow A(x)$.

Lemma 1.20. Suppose $X$ is a gerbe over the algebraic space $Y$, and $A \rightarrow$ $X$ is an algebra. Then there exists a sheaf of $\mathscr{O}_{Y}$-algebras $B$, and an 
isomorphism $\left.A \cong B\right|_{X}$ if and only if the inertia representation $I_{X} \rightarrow$ Aut $_{X}(A)$ is trivial.

Similarly, if $X$ is a connected gerbe over the Deligne-Mumford stack $Y$, then an algebra $A$ over $X$ descends to $B$ over $Y$, if and only if the inertia representation restricts to a trivial homomorphism $I_{X}^{\circ} \rightarrow \underline{\mathrm{Aut}}_{X}(A)$.

In either case, $A$ is representable or of finite type if and only if $B$ is.

We can pull back the sheaf of algebras $A$ over $X$, via the structure morphism $I_{X} \rightarrow X$, to obtain the sheaf of algebras $\left.A\right|_{I_{X}}$. This sheaf of algebras is endowed with a tautological automorphism, induced from (6). The algebra of invariants for this automorphism we shall denote by $A_{I_{X}}^{f i x}$.

The following statement is somewhat tautological, and holds more generally than for algebras.

Proposition 1.21. Suppose that $A$ is a representable algebra over the algebraic stack $X$. Then the inertia stack of $A$ is naturally identified with $A_{I_{X}}^{f i x}$. In particular, $I_{A}$ is a representable algebra over $I_{X}$.

Proof. We have a commutative diagram of algebraic stacks

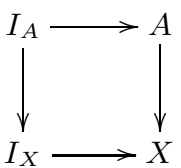

which identifies $I_{A}$ with a substack of $\left.A\right|_{I_{X}}$. The algebra $\left.A\right|_{I_{X}}$ is the stack of triples $(x, \varphi, a)$, where $x$ is an object of $X, \varphi$ is an automorphism of $x$, and $a \in A(x)$ is an object of $A$ lying over $x$. Such a triple is in $I_{A}$, if and only if $\varphi \in \operatorname{Aut}(x)$ is in the subgroup $\operatorname{Aut}(a) \subset \operatorname{Aut}(x)$. This is equivalent to $\varphi$ fixing $a$ under the action of $\operatorname{Aut}(x)$ on $A(x)$. This is the claim.

In fact, the fibre of $I_{A}$ over the object $x$ of $X$ is equal to

$$
I_{A}(x)=\left\{(\varphi, a) \in \operatorname{Aut}(x) \times A(x) \mid \varphi^{*}(a)=a\right\} .
$$

The fibre of $I_{A}(x)$ over $\varphi \in \operatorname{Aut}(x)$ is the subalgebra $A(x)^{\varphi} \subset A(x)$, and the fibre of $I_{A}(x)$ over $a \in A(x)$ is the subgroup $\operatorname{Stab}_{\operatorname{Aut}(x)}(a) \subset \operatorname{Aut}(x)$.

\section{Algebra bundles}

Definition 1.22. We call a finite type algebra $A \rightarrow X$ an algebra bundle, if the underlying $\mathscr{O}_{X}$-module is locally free (necessarily of finite rank).

When studying finite type algebras over finite type stacks $X$, we may, after passing to a locally closed stratification of $X$, assume that the finite type algebra is an algebra bundle.

Definition 1.23. Let $A \rightarrow X$ be a finite type algebra over the algebraic stack $X$. The stratification $X^{\prime} \subset X$ of Proposition 1.5 is characterized by the property that an $X$-scheme $S$ factors through $X^{\prime}$ if and only if $\left.A\right|_{S}$ is an algebra bundle. The pullback $A^{\prime}=\left.A\right|_{X^{\prime}}$ is an algebra bundle, and the induced stratification $A^{\prime} \rightarrow A$ of $A$ is called the rank stratification of A. 
Remark 1.24. By considering the representation of $A$ on itself by left multiplication, we see that every algebra bundle is a sheaf of subalgebras of the algebra $\operatorname{End}(V)$ of endomorphisms of a vector bundle $V$ over the stack $X$.

\section{Central idempotents}

Lemma 1.25. The centre of an algebra bundle is a finite type algebra.

Proof. The centre of $A$ is the kernel of the $\mathscr{O}_{X}$-linear homomorphism of vector bundles $A \rightarrow \underline{\text { End }}_{\mathscr{O}_{X}}(A)$, given by $a \mapsto[a, \cdot]$. As such, it is a locally coherent representable sheaf.

Thus, if $A \rightarrow X$ is a finite type algebra over a finite type stack, after passing to a locally closed stratification of $X$, we may assume that $A$ is an algebra bundle, whose centre is an algebra bundle.

If $A \rightarrow X$ is a commutative algebra bundle, then $\pi: Y=\operatorname{Spec}_{X} A \rightarrow$ $X$ is a finite flat representable morphism, and $A=\pi_{*} \mathscr{O}_{Y}$. In fact, the category of commutative algebra bundles over $X$ is equivalent to the category of finite flat representable stacks over $X$.

For a commutative finite type algebra $A \rightarrow X$, we denote the stack of idempotents in $A$ by $E(A)$.

Lemma 1.26. Suppose $A$ is a commutative algebra bundle over the algebraic stack $X$. Then the structure morphism $E(A) \rightarrow X$ is affine, of finite type, and étale. In particular, there exists a non-empty open substack $U \subset X$, such that $\left.E(A)\right|_{U}=E\left(\left.A\right|_{U}\right)$ is finite étale over $U$.

Proof. We reduce to the case where $X$ is a scheme, and then quote Lemme (18.5.3) of EGA IV 9.

By this lemma, when studying the centre of finite type algebras over the finite type stack $X$, we may, after passing to a stratification of $X$, assume that the stack of central idempotents is finite étale over $X$.

\section{Primitive idempotents}

Recall that a non-zero idempotent $e$ is called primitive, if whenever $e=$ $e_{1}+e_{2}$, for orthogonal idempotents $e_{1}, e_{2}$, then necessarily $e_{1}=0$ or $e_{2}=0$.

In a finite-dimensional commutative algebra over a field, the following is true:

(i) every idempotent is in a unique way (up to order of the summands) a sum of orthogonal primitive idempotents, this is the primitive decomposition,

(ii) orthogonal idempotents have disjoint primitive decompositions,

(iii) distinct primitive idempotents are orthogonal to each other,

(iv) the primitive idempotents add up to 1 . 
Thus, the idempotents are in bijection with the subsets of the (finite) set of primitive idempotents.

Let $A \rightarrow X$ be a finite type algebra.

Definition 1.27. An idempotent local section $e: U \rightarrow A$ of $A \rightarrow X$ is primitive, if it gives rise to a primitive idempotent in the fiber of $A$ over every geometric point of $U$.

Suppose $A=\pi_{*} \mathscr{O}_{Y}$ is a commutative algebra bundle, and $e$ an idempotent global section. Let $Y_{1} \subset Y$ be the open and closed substack defined by the equation $e=1$. Then $e$ is primitive, if and only if the geometric fibres of $Y_{1} \rightarrow X$ are connected. As the function counting the number of connected components of the fibres is lower semi-continuous, the subset of $X$ where $e$ is primitive, is closed. In general, this subset is not open. Therefore, when studying primitive idempotents, we assume that $E(A) \rightarrow X$ is finite étale.

Lemma 1.28. Let $A \rightarrow X$ be a commutative algebra bundle, with finite étale stack of idempotents $E(A) \rightarrow X$. There is an open and closed substack $P E(A) \subset E(A)$, such that an idempotent local section factors through $P E(A)$ if and only if it is primitive.

Proof. We may assume that $E(A) \rightarrow X$ is constant. Then the multiplication operation and the partially defined addition operation on $E(A)$ are also constant. The claim follows.

Definition 1.29. Let $A \rightarrow X$ be an algebra bundle, with centre $Z \rightarrow X$. Let $Z E(A)$ be the stack of idempotents in $Z$, in other words the stack of central idempotents in $A$. Assume that $Z E(A) \rightarrow X$ is finite étale. The substack of primitive idempotents in $Z E(A)$ is denoted by $P Z E(A)$, and called the stack of primitive central idempotents of $A$. It is finite étale over $X$. The degree of $P Z E(A) \rightarrow X$ is called the central rank of A.

If $X$ is connected, the number of connected components of $P Z E(A)$ is the split central rank of $A$. More precisely, the partition of the central rank given by the degrees of the connected components of $P Z E(A)$ is called the central type of $A$. (So the split central rank is the length of the type.)

Remark 1.30. Let $X$ be connected, and let $A \rightarrow X$ be a commutative finite type algebra, with finite étale stack of idempotents $E(A) \rightarrow X$. Then there is a one-to-one correspondence between the connected components of $P E(A)$ and the primitive idempotents in the algebra of global sections $\Gamma(X, A)$. 


\section{The degree stratification}

Let $k$ be a field and $A$ a finite-dimensional $k$-algebra. The rank $r$ of $A$ is the dimension of $A$ as a $k$-vector space. For an element $a \in A$, we define its degree to be the dimension of the commutative subalgebra $k[a] \subset A$. It is equal to the degree of the minimal polynomial of $a$, i.e., the monic generator of the kernel of the algebra map $k[x] \rightarrow A$, defined by $x \mapsto a$.

Now let $A$ be an algebra bundle of rank $r$ over the algebraic stack $X$, and $a \in A(S)$ a local section of $A$ over an $X$-scheme $S$.

Definition 1.31. If the cokernel (as a homomorphism of $\mathscr{O}_{S}$-modules) of the morphism of $\mathscr{O}_{S}$-algebras $\mathscr{O}_{S}[x] \rightarrow A_{S}$, defined by $x \mapsto a$ is flat over $\mathscr{O}_{S}$, we say that $a$ is strict, and we call the rank of the image of $\mathscr{O}_{S}[x] \rightarrow A$ the degree of $a$.

If $f(x) \in \mathscr{O}_{S}[x]$ is the characteristic polynomial of $a$, the morphism $\mathscr{O}_{S}[x] \rightarrow A$ factors through $\mathscr{O}_{S}[x] /(f)$, by the theorem of Caley-Hamilton. Hence the cokernel of $\mathscr{O}_{S}[x] \rightarrow A$ is actually a cokernel of a homomorphism of vector bundles, and hence coherent. The condition that this cokernel be flat is equivalent to it being locally free. It implies that forming the image of $\mathscr{O}_{S}[x] \rightarrow A$ commutes with base change, and that this image, denoted $\mathscr{O}_{S}[a]$, is also locally free.

Proposition 1.32. For every $n=1, \ldots, r$, there exists a locally closed substack $A_{n} \subset A$ with the property that a local section $a \in A(S)$ factors through $A_{n}(S)$ if and only if a is strict of degree $n$. The $A_{n}$ are pairwise disjoint and their disjoint union

$$
A^{\text {strat }}=\coprod_{n=1}^{r} A_{n}
$$

maps surjectively to $A$. The section $a \in A(S)$ factors through $A^{\text {strat }} \rightarrow A$ if and only if it is strict.

Proof. Consider the tautological section $\Delta$ of the pullback of $A$ via the structure map $A \rightarrow X$. It gives rise to a morphism of $\mathscr{O}_{A}$-algebras $\mathscr{O}_{A}[x] \rightarrow$ $A_{A}$. Then $A^{\text {strat }}$ is given by the flattening stratification of its cokernel, and $A_{n} \subset A^{\text {strat }}$ is the component where the cokernel has rank $r-n$.

We call the stratification $A^{\text {strat }} \rightarrow A$ the degree stratification of $A$.

\section{Semi-simple elements}

Let $k$ be an algebraically closed field, and $A$ a finite-dimensional $k$-algebra. Recall that an element $a \in A$ is semi-simple, if the following equivalent conditions are satisfied:

(i) the map $A \rightarrow A$ given by left multiplication by $a$ is diagonalizable,

(ii) the minimal polynomial $f \in k[x]$ of $a$ is separable, i.e., satisfies $\left(f, f^{\prime}\right)=1$,

(iii) the commutative subalgebra $k[a] \subset A$ is reduced, or, equivalently, étale over $k$. 
Definition 1.33. Let $A \rightarrow X$ be an algebra bundle. A local section $a \in A(S)$, for an $X$-scheme $S$, is called semi-simple, if it is strict, and for every geometric point $s \in S$, the element induced by $a$ in $A(s)$ is semi-simple.

For example, an idempotent section $e \in A(S)$ is semi-simple over the open subset of $S$, where $e$ is neither 0 nor 1 (in a commutative algebra bundle, this subset is also closed).

Assuming $a \in A(S)$ is strict, $a$ is semi-simple if and only if the geometric fibres of the finite flat $S$-scheme $\operatorname{Spec}_{S} \mathscr{O}_{S}[a]$ are unramified. This condition is equivalent to $\operatorname{Spec}_{S} \mathscr{O}_{S}[a]$ being unramified, hence étale over $S$.

The semi-simple sections of $A$ form a subsheaf $A^{s s} \subset A$.

Proposition 1.34. Let $A \rightarrow X$ be an algebra bundle over the algebraic stack $X$. Then $A^{\text {ss }}$ is an algebraic stack with a representable structure morphism of finite type $A^{s s} \rightarrow X$.

Proof. In fact, $A^{s s} \subset A^{\text {strat }}$ is the open substack defined by the condition that the finite flat representable morphism $\operatorname{Spec}_{A} \mathscr{O}_{A}[\Delta] \rightarrow A$ is unramified. Thus, we have a factorization of the monomorphism $A^{s s} \rightarrow A$ as

$$
A^{\text {ss }} \stackrel{\text { open immersion }}{\longrightarrow} A^{\text {strat }} \stackrel{\text { stratification }}{\longrightarrow} A .
$$

Thus, $A^{s s} \subset A$ is a constructible substack.

\section{The semi-simple centre}

For a commutative finite dimensional algebra over an algebraically closed field, we have

(i) the primitive idempotents are linearly independent,

(ii) an element is semi-simple if and only if it is a linear combination of primitive idempotents.

We need a version of this statement for algebra bundles.

Proposition 1.35. Let $A \rightarrow X$ be a commutative algebra bundle whose stack of idempotents is finite étale, and let $Y \rightarrow X$ be the finite flat cover corresponding to $A$. There is a canonical finite flat morphism of X-stacks $Y \rightarrow P E(A)$. Over every geometric point $x$ of $X$, this morphism maps each point in the fibre $Y_{x}$ to the characteristic function of its connected component in $Y_{x}$ (which is a primitive idempotent in $\left.A\right|_{x}$ ). Dually, we obtain a strict monomorphism of algebra bundles

$$
\pi_{*} \mathscr{O}_{P E(A)} \longrightarrow A
$$

where $\pi: P E(A) \rightarrow X$ is the structure map.

The induced morphism

$$
\left(\pi_{*} \mathscr{O}_{P E(A)}\right)^{\text {strat }} \longrightarrow A^{\text {strat }}
$$

factors through the open substack $A^{s s} \subset A^{\text {strat }}$, and induces a surjective closed immersion of algebraic stacks $\left(\pi_{*} \mathscr{O}_{P E(A)}\right)^{\text {strat }} \rightarrow A^{\text {ss }}$. 
Proof. Consider the finite étale cover of primitive idempotents $\pi: P E(A) \rightarrow$ $X$. We have a tautological global section $e$ of $\left.A\right|_{P E(A)}$, and $a \mapsto a e$ defines a homomorphism of $\mathscr{O}_{P E(A)}$-modules $\left.\mathscr{O}_{P E(A)} \rightarrow A\right|_{P E(A)}$. Pushing forward with $\pi$ and composing with the trace map $\pi_{*}\left(\left.A\right|_{P E(A)}\right) \rightarrow A$ defines the morphism of algebra bundles over $X$

$$
\pi_{*} \mathscr{O}_{P E(A)} \longrightarrow A \text {. }
$$

It is a strict monomorphism of vector bundles, because it is injective over every geometric point, by Fact (i), above. Dually, we obtain a morphism of $X$-stacks $Y \rightarrow P E(A)$, which is the morphism described in the statement of the proposition. It is flat, because $P E(A)$ is étale over $X$, and flatness can be checked étale locally.

Passing to the degree stratification commutes with strict monomorphisms of algebra bundles, so we have a cartesian diagram of $X$-stacks

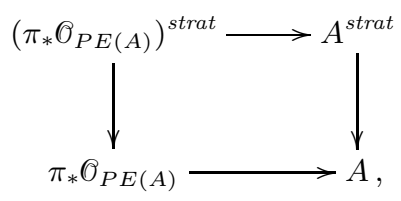

which shows that $\left(\pi_{*} \mathscr{O}_{P E(A)}\right)^{\text {strat }} \rightarrow A^{\text {strat }}$ is a closed immersion. The facts that this closed immersion factors through $A^{s s} \subset A^{\text {strat }}$, and is surjective onto $A^{s s}$ can be checked over the geometric points of $X$, where it follows from Fact (ii), above.

\section{Permanence of rank and split rank}

Proposition 1.36. Let $A \hookrightarrow A^{\prime}$ be a monomorphism of commutative finite type algebras with finite étale stacks of idempotents over the connected stack $X$. Denote the ranks of $A$ and $A^{\prime}$ by $n$ and $n^{\prime}$, and the split ranks by $k$ and $k^{\prime}$, respectively. Then $n \leq n^{\prime}$ and $k \leq k^{\prime}$. Moreover,

(i) if $A^{\prime}$ admits a semi-simple global section which does not factor through the fiber $\left.A\right|_{x}$ for all points of $x$, then $n<n^{\prime}$,

(ii) if $A^{\prime}$ admits an idempotent global section, which is not in $A$, then $k<k^{\prime}$.

Proof. The monomorphism $A \hookrightarrow A^{\prime}$ induces an open and closed embedding of finite étale $X$-stacks $E(A) \hookrightarrow E\left(A^{\prime}\right)$. Every idempotent $e$ in $A$ can be decomposed uniquely into a sum of orthogonal primitive idempotents in $A^{\prime}$. Let us call this the primitive decomposition of $e$ in $A^{\prime}$. Consider the correspondence $Q \subset P E(A) \times{ }_{X} P E\left(A^{\prime}\right)$ defined by

$\left(e, e^{\prime}\right) \in Q \quad \Longleftrightarrow \quad e^{\prime}$ partakes in the primitive decomposition of $e$ in $A^{\prime}$ $\Longleftrightarrow e e^{\prime}=e^{\prime}$.

One shows that $Q$ is a finite étale cover of $X$ locally in the étale topology of $X$, reducing to the case where both $E(A)$ and $E\left(A^{\prime}\right)$ are trivial covers. 
By properties of the primitive decomposition, the projection $Q \rightarrow P E(A)$ is surjective, and the projection $Q \rightarrow P E\left(A^{\prime}\right)$ is injective. Thus we have

$$
n=\operatorname{deg} P E(A) \leq \operatorname{deg} Q \leq \operatorname{deg} P E\left(A^{\prime}\right)=n^{\prime} .
$$

If $n=n^{\prime}$, then both $Q \rightarrow P E(A)$ and $Q \rightarrow P E\left(A^{\prime}\right)$ are isomorphisms, showing that $P E(A)=P E\left(A^{\prime}\right)$. By Proposition 1.35 this implies that the morphism $A^{s s} \rightarrow\left(A^{\prime}\right)^{s s}$ is surjective. This proves (i).

We can repeat the argument for the algebras of global sections $\Gamma(X, A) \hookrightarrow$ $\Gamma\left(X, A^{\prime}\right)$. We deduce that $k \leq k^{\prime}$, and if $k=k^{\prime}$, every primitive idempotent in $\Gamma(X, A)$ remains primitive in $\Gamma\left(X, A^{\prime}\right)$, and every primitive idempotent of $\Gamma\left(X, A^{\prime}\right)$ is in $\Gamma(X, A)$. We deduce that $\Gamma(X, A)$ and $\Gamma\left(X, A^{\prime}\right)$ have the same idempotents, which proves (ii).

\section{Families of idempotents}

Definition 1.37. For a finite type algebra $A \rightarrow X$, we denote by $E_{n}(A) \rightarrow$ $X$ the stack of $n$-tuples of non-zero idempotents in $A$, which are pairwise orthogonal, and add up to unity. We call sections of $E_{n}(A)$ also complete sets of orthogonal idempotents.

Note that the family members of the sections of $E_{n}(A)$ need not be central.

The stack $E_{n}(A)$ is algebraic, and of finite type over $X$.

For $n=0$, the stack $E_{0}(A)$ is empty, unless $A=0$, in which case it is identified with $X$. For $n=1$, the stack $E_{1}(A)$ contains exactly the unit in $A$ (so is identified with $X$ ), unless $A=0$, in which case $E_{1}(A)$ is empty.

\section{Group of units}

Let $A \rightarrow X$ be a finite type algebra over the algebraic stack $X$. The subsheaf of units $A^{\times} \subset A$ is defined by

$$
A^{\times}(x)=\{a \in A(x) \mid \exists b \in A(x): a b=b a=1\},
$$

for every object $x$ of $X$. We can see that $A^{\times}$is a (relative) affine group scheme over $X$, by writing it as the fibered product

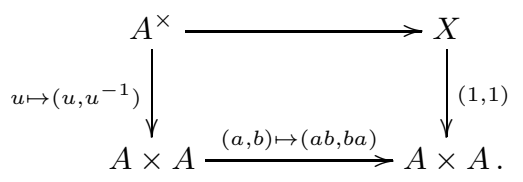

if $A$ is an algebra bundle, the subsheaf $A^{\times} \subset A$ is represented by an open substack, because in this case, a local section $a$ is invertible if and only if the determinants of left and right multiplication by $a$ on $A$ do not vanish. We conclude that if $A$ is an algebra bundle, $A^{\times}$is smooth over $X$ with geometrically connected fibers. For the general case, this implies that $A^{\times}$is an affine group scheme with smooth and geometrically connected fibers over $X$. 
A similar argument using the determinant proves that if $A \rightarrow B$ is a strict monomorphism of algebra bundles, we have $A^{\times}=A \cap B^{\times}$. Also, if $A$ is an algebra bundle, any morphism $A \rightarrow B$ to another algebra bundle is determined by its restriction to $A^{\times}$.

\subsection{Algebroids}

Definition 1.38. A algebroid is a triple $(X, A, \iota)$, where $X$ is an algebraic stack, $A$ is a finite type algebra over $X$, and $\iota: A^{\times} \rightarrow I_{X}$ is a homomorphism of sheaves of groups over $X$, which identifies $A^{\times}$with an open substack of $I_{X}$. Moreover, we require that the diagram of groups over $X$

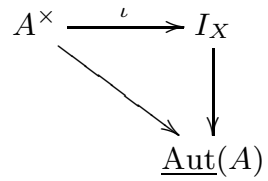

commutes. Here, the map $A^{\times} \rightarrow \underline{\operatorname{Aut}}_{X}(A)$ associates to a unit $u$ of $A$ the inner automorphism $x \mapsto u x u^{-1}$. The vertical map $I_{X} \rightarrow \underline{\operatorname{Aut}}_{X}(A)$ is the inertia representation (6). If $\iota$ is an isomorphism, we call $(X, A, \iota)$ a strict algebroid.

We will usually abbreviate the triple $(X, A, \iota)$ to $X$, and write $A_{X}$ for $A$, if we need to specify the algebra. We call the commutativity of (7) the algebroid property.

For an explanation of the terminology, see Remark 1.44

The condition that $\iota$ is an open immersion implies that $A^{\times}$represents the subsheaf $I_{X}^{\circ} \subset I_{X}$ of connected components of the identity. (Over a field, $A^{\times}$is connected, and $A^{\times} \rightarrow I_{X}$ being an open immersion implies that $I_{X} / A^{\times}$is étale. These conditions characterize the connected component over a field.) In particular, if $X$ is a connected gerbe over a Deligne-Mumford stack $S$, then the relative inertia $I_{X / S}$ is necessarily equal to $I_{X}^{\circ}$, and hence $A^{\times}$is identified with $I_{X / S}$.

If $A$ is an algebra bundle, $A^{\times}$, and therefore also $I_{X}^{\circ}$, is an affine smooth (relative) group scheme over $X$. Hence $X$ admits a coarse DeligneMumford stack $X \rightarrow \bar{X}$, which is uniquely determined by being a DeligneMumford stack, and $X \rightarrow \bar{X}$ being a connected gerbe. Moreover, $A^{\times}$is then identified with the relative inertia group $I_{X / \bar{X}} \subset I_{X}$, and we have a short exact sequence of relative group schemes

$$
\left.1 \longrightarrow A^{\times} \stackrel{\iota}{\longrightarrow} I_{X} \longrightarrow I_{\bar{X}}\right|_{X} \longrightarrow 1
$$

over $X$. In case $(X, A)$ is a strict algebroid, $I_{X}^{\circ}=I_{X}$, and $\bar{X}$ is an algebraic space, in fact the coarse moduli space of $X$.

In many cases, the algebroid property is automatic:

Proposition 1.39. Consider a triple $(X, A, \iota)$, where $X$ is an algebraic stack, $A$ is an algebra bundle over $X$, and $\iota: A^{\times} \rightarrow I_{X}$ is a homomorphism of sheaves of groups over $X$, which identifies $A^{\times}$with an open substack of $I_{X}$. Then (7) commutes, so that $(X, A, \iota)$ is an algebroid. 
Proof. As $\iota: A^{\times} \rightarrow I_{X}$ is defined over $X$, the homomorphism $\iota$ is equivariant with respect to the inertia action. The proof now combines the facts that $\iota$ is a monomorphism, that the inertia action on $I_{X}$ is the inner action, and the fact that a morphism of algebra bundles is determined by its restriction to units.

In fact, let $a \in A^{\times}$. To show that ${ }^{a}(\cdot)=\iota(a)$, as automorphism of $A$, it suffices to show that for all $b \in A^{\times}$, we have ${ }^{a} b=\iota(a) \cdot b$. We can check this after applying $\iota$, so it suffices that $\iota\left({ }^{a} b\right)=\iota(\iota(a) \cdot b)$, or ${ }^{\iota(a)} \iota(b)=\iota(a) \cdot \iota(b)$, which is true.

Example 1.40. (algebroid underlying a linear stack) Let $\mathfrak{X}$ be a linear algebraic stack with underlying algebraic stack $X$, and let $A \rightarrow X$ be the universal sheaf of endomorphisms of Remark 1.10. Then automorphisms are invertible endomorphisms, so we use for $\iota$ the tautological identification $A^{\times}=I_{X}$.

The inertia representation being the inverse of the pullback action, it is, indeed, given by (left) inner automorphisms.

We call $(X, A)$ the algebroid underlying the linear algebraic stack $\mathfrak{X}$. It is a strict algebroid.

Example 1.41. Consider the linear stack of vector bundles $\mathfrak{V e c t}$, Example 1.12. The underlying algebroid consists of the disjoint union of the quotient stacks $\mathrm{GL}_{n} \backslash M_{n \times n}$, given by the adjoint representations, for $n \geq 0$. Thus, in passing from the linear stack to the underlying algebroid, we discard all $M_{m \times n}$, for $m \neq n$, and for $m=n$, restrict the left-right biaction of $\mathrm{GL}_{n}$ on $M_{n \times n}$ to the (left only) adjoint action. Thus we remove exactly the information which we consider non-local, see Remark 1.15.

Example 1.42. (classifying algebroid) Let $A \rightarrow X$ be an algebra bundle over a Deligne-Mumford stack $X$. Let $A^{\times}$act on $A$ from the left by inner automorphisms. Then $A^{\times} \backslash A$ is an algebra bundle over the relative classifying stack $Y=B_{X} A^{\times}$. We have an exact sequence of group schemes over $Y$

$$
\left.1 \longrightarrow I_{Y / X} \longrightarrow I_{Y} \longrightarrow I_{X}\right|_{Y} \longrightarrow 1
$$

where $I_{Y / X}=A^{\times} \backslash A^{\times}$. As $I_{X} \rightarrow X$ is unramified, $I_{Y / X} \rightarrow I_{Y}$ is an open embedding, and so $\left(Y, A^{\times} \backslash A\right)$ is an algebroid. If $X$ is a space, $\left(Y, A^{\times} \backslash A\right)$ is a strict algebroid.

Remark 1.43. (algebroids which are trivial gerbes are classifying algebroids) Let $(X, A)$ be an algebroid, such that $A$ is an algebra bundle over $X$, and therefore $X$ is a connected gerbe over the Deligne-Mumford stack $S$, with $A^{\times}=I_{X / S}$. Suppose the gerbe $X \rightarrow S$ admits a section $x: S \rightarrow X$. Via $x$, we pull back $A$ to a bundle of algebras $C$ over $S$. We claim that $(X, A)$ is canonically isomorphic to $\left(B C^{\times}, C^{\times} \backslash C\right)$. 
In fact, because $X$ is a gerbe over $S$, the section $x: S \rightarrow X$ is a universal principal $x^{*} I_{X / S}$-bundle. The pullback diagram

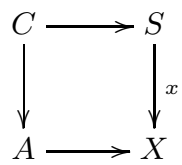

shows that $C$ is an $x^{*} I_{X / S}$-bundle over $A$. Hence, $A=C / x^{*} I_{X / S}$. Via the isomorphism $\iota: C^{\times} \rightarrow x^{*} I_{X / S}$, the action of $x^{*} I_{X / S}$ on $C$ is identified with the action by left inner automorphisms. This follows from the algebroid property of $(X, A)$, and proves the claim.

Remark 1.44. If $X \rightarrow \operatorname{Spec} R$ is a gerbe, any strict algebroid over $X$ can be promoted to a linear algebraic stack, whose underlying algebraic stack is $X$.

More generally, there exists a notion of relative linear algebraic stack, where the base $R$ is replaced by an arbitrary scheme (or algebraic space). Then every strict algebroid $(X, A)$ where $X$ is a gerbe over a space $S$ becomes naturally a linear algebraic stack over $S$. These types of linear algebraic stacks occur naturally in the theory of deformation quantization, where they were introduced by Kontsevich under the name of 'stack of algebroids', see [13. In [loc. cit.], one can also find a description of these stacks of algebroids in terms of cocycles (compare also [7]).

Thus our notion of algebroid is a natural generalization of Kontsevich's notion of stack of algebroids. This justifies our terminology.

Loosely speaking, algebroids are stacks which are linear over their coarse Deligne-Mumford stack.

Example 1.45 (schemes as algebroids). Every Deligne-Mumford stack $Z$ is an algebroid via the definition $A_{Z}=0_{Z}$. There is no natural way to enhance the algebroid $\left(Z, 0_{Z}\right)$ to a linear algebraic stack, unless $Z=\operatorname{Spec} R$ is the final scheme. This exhibits one way in which algebroids are more flexible than linear algebraic stacks.

Example 1.46 (algebroids over quotient stacks). Let $X=G \backslash Y$ be a quotient stack. A finite type algebra $A$ over $X$ is given by a finite type algebra $B$ over $Y$, together with a lift of the $G$-action on $X$ to an action on $B$ by algebra automorphisms. The inertia stack of $X$ is naturally identified with $G \backslash \operatorname{Stab}_{G} Y$. Thus, $(X, A)$ becomes an algebroid, if we specify a $G$ equivariant open embedding of $Y$-group schemes $\iota: B^{\times} \rightarrow \operatorname{Stab}_{G} Y$. If $B$ is not an algebra bundle, we also need to require that ${ }^{u} b=\iota(u) \cdot b$, for all $u \in B^{\times}, b \in B$. 


\section{Morphisms of algebroids}

Definition 1.47. We call a morphism of algebraic stacks $f: X \rightarrow Y$ inert, if the diagram of stacks

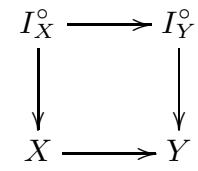

is cartesian. If $\left.I_{X} \rightarrow I_{Y}\right|_{X}$ is an isomorphism, we call $f$ strictly inert.

The connected component $I_{Y}^{\circ}$ is not necessarily an algebraic stack, but if it is, then so is $I_{X}^{\circ}$, if $X \rightarrow Y$ is inert. We will only apply this concept when $Y$ is an algebroid, so that $I_{Y}^{\circ}$ is representable over $Y$.

The basic facts about inert morphisms are:

(i) Every inert morphism of algebraic stacks is Deligne-Mumford representable, because its relative inertia group scheme is unramified.

(ii) Every base change of an inert morphism of algebraic stacks is inert.

(iii) Every monomorphism of algebraic stacks (in particular every locally closed immersion and every stratification) is (strictly) inert.

(iv) Being inert is local in the étale topology of the target.

(v) Every morphism of Deligne-Mumford stacks is inert.

Base changes of morphisms of Deligne-Mumford stacks are, in fact, the only inert morphisms, at least up to stratifications:

Proposition 1.48. Suppose $X \rightarrow Y$ is an inert morphism of algebraic stacks, and suppose $I_{Y}^{\circ}$ (and hence also $I_{X}^{\circ}$ ) is smooth and representable over $X$, so that we have coarse Deligne-Mumford stacks $\bar{X}, \bar{Y}$, and an induced morphism $\bar{X} \rightarrow \bar{Y}$. Then the diagram

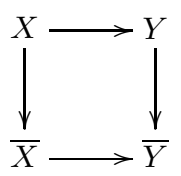

is cartesian.

Proof. To prove that the morphism of gerbes $\left.X \rightarrow Y\right|_{\bar{X}}$ over $\bar{X}$ is an isomorphism, we may pass to an étale cover $\bar{X}^{\prime}$ of $X$ and assume that the gerbe $X$ is trivial. Then $X=B_{\bar{X}} G$, where $G$ is the pullback of $I_{X}^{\circ}$ to $\bar{X}$ via a trivializing section. Moreover, $\left.Y\right|_{\bar{X}}=B_{\bar{X}} H$, where $H$ is the pullback of $\left.I_{Y}^{\circ}\right|_{X}$ to $\bar{X}$ via the same trivializing section. Since $\left.I_{X}^{\circ} \rightarrow I_{Y}^{\circ}\right|_{X}$ is an isomorphism, so is $G \rightarrow H$, and hence $\left.X \rightarrow Y\right|_{\bar{X}}$. 
Definition 1.49. A morphism of algebroids $X \rightarrow Y$ is a pair $(f, \varphi)$, where $f: X \rightarrow Y$ is a morphism of algebraic stacks, and $\varphi: A_{X} \rightarrow A_{Y}$ is a morphism of algebras over $f$, such that the diagram

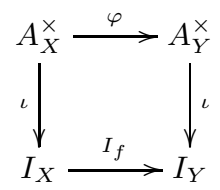

commutes.

The morphism $(f, \varphi)$ is a representable morphism of algebroids if $\varphi:\left.A_{X} \rightarrow A_{Y}\right|_{X}$ is a monomorphism of sheaves of algebras over $X$. (This implies that $f$ is Deligne-Mumford representable.)

The morphism $(f, \varphi)$ is inert, if $\varphi:\left.A_{X} \rightarrow A_{Y}\right|_{X}$ is an isomorphism of finite type algebras. (This implies that $f$ is inert.)

There is a natural notion of 2-morphism of algebroid, which makes algebroids into a 2-category.

Remark 1.50 (inert morphisms in the case of algebra bundles). Suppose $\left(X, A_{X}\right) \rightarrow\left(Y, A_{Y}\right)$ is a morphism of algebroids, where $A_{X}$ and $A_{Y}$ are algebra bundles. If $X \rightarrow Y$ is an inert morphism of algebraic stacks, then we automatically have $A_{X}^{\times}=\left.A_{Y}^{\times}\right|_{X}$ and hence $A_{X}=\left.A_{Y}\right|_{X}$, and so $\left(X, A_{X}\right) \rightarrow\left(Y, A_{Y}\right)$ is an inert morphism of algebroids.

Remark 1.51 (strict algebroids and representable morphisms). Suppose $(X, A)$ is a strict algebroid, and $f: Y \rightarrow X$ a representable morphism of algebraic stacks. If $\left.B \subset A\right|_{Y}$ is a finite type subalgebra, such that $\iota\left(B^{\times}\right)=I_{Y} \cap \iota\left(\left.A^{\times}\right|_{Y}\right)$ inside $\left.I_{X}\right|_{Y}$, then $(Y, B)$ is a strict algebroid with a representable morphism $(Y, B) \rightarrow(X, A)$. (The algebroid condition for $(Y, B)$ is automatic. $)$

Every strict algebroid over $f: Y \rightarrow X$ and $(X, A)$ comes about in this way.

Remark 1.52 (pullbacks). Suppose $\left(X, A_{X}\right)$ is an algebroid, and $Y \rightarrow$ $X$ an inert morphism of algebraic stacks. In this case, $Y$ admits a unique algebroid $A_{Y}$, endowed with an inert morphism of algebroids $\left(Y, A_{Y}\right) \rightarrow$ $\left(X, A_{X}\right)$. In fact, $A_{Y}=\left.A_{X}\right|_{Y}$.

If $\left(X, A_{X}\right)$ is a strict algebroid, then the morphism $Y \rightarrow X$ is necessarily strictly inert, and $\left(Y, A_{Y}\right)$ is necessarily a strict algebroid. We call $\left(Y, A_{Y}\right) \rightarrow\left(X, A_{X}\right)$ is a strictly inert morphism of strict algebroids.

Definition 1.53. We call a morphism of algebroids $\left(X, A_{X}\right) \rightarrow\left(Y, A_{Y}\right)$

(i) a vector bundle,

(ii) a principal homogeneous $G$-bundle, for an algebraic group $G$, 
(iii) a fibre bundle with group $G$ and fibre $F$,

(iv) a locally closed immersion,

(v) a stratification,

if it is inert, and the underlying morphism of algebraic stacks $X \rightarrow Y$ has the indicated property.

Remark 1.54 (fibered products). Fibered products of algebroids exist, and commute with fibered products of underlying stacks and underlying algebras. Fibered products of strict algebroids are strict algebroids. The algebroid underlying a fibered product of linear algebraic stacks is equal to the fibered product of the underlying algebroids.

Proposition 1.55. Suppose $(X, A) \rightarrow(Y, B)$ is a morphism of algebroids where $X \rightarrow Y$ is a stratification of algebraic stacks, then there exists a stratification of algebroids $\left(X^{\prime}, A^{\prime}\right) \rightarrow(X, A)$, such that the composition $\left(X^{\prime}, A^{\prime}\right) \rightarrow(Y, B)$ is also a stratification of algebroids.

If $(Y, B)$ is strict, then $\left(X^{\prime}, A^{\prime}\right) \rightarrow(X, A)$ and $\left(X^{\prime}, A^{\prime}\right) \rightarrow(Y, B)$ are stratifications of strict algebroids.

Proof. Passing to the rank stratifications of $A$ and $B$, we obtain a commutative diagram of algebroids

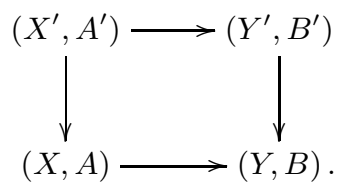

The upper horizontal morphism is an inert morphism of algebroids, because $A^{\prime}$ and $B^{\prime}$ are algebra bundles, by Remark 1.50 The claim follows.

\section{Algebroid Inertia}

Remark 1.56 (Inertia). Let $(X, A)$ be an algebroid. Then $\left(I_{X}, I_{A}\right)$ is another algebroid, which we call the algebroid inertia of $(X, A)$. In fact, $I_{A}=\left(\left.A\right|_{I_{X}}\right)^{f i x}$, the subalgebra of $\left.A\right|_{I_{X}}$ of elements invariant under the tautological automorphism induced by the inertia action of $I_{X}$ on $A$. The subgroup of units is $\left(\left.A^{\times}\right|_{I_{X}}\right)^{f i x}$, and we have a cartesian diagram

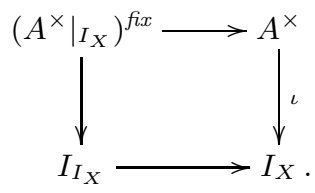

If $(X, A)$ is a strict algebroid, then so is $\left(I_{X}, I_{A}\right)$. 
Remark 1.57 (Induced algebroid structure on the algebra). Let $(X, A)$ be an algebroid. Let $\left(\left.A\right|_{A}\right)^{f i x}$ be the subalgebra of the pullback $\left.A\right|_{A}$ of elements commuting with the tautological section of $\left.A\right|_{A}$. (This is equal to the space of commuting pairs in $A \times_{X} A$ ). The subgroup of units is $\left(\left.A^{\times}\right|_{A}\right)^{f i x}$, and we have a cartesian diagram

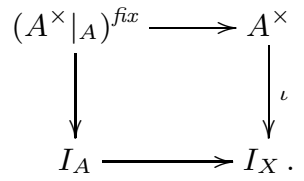

This proves that $\left(A,\left(\left.A\right|_{A}\right)^{f i x}\right)$ is an algebroid over $A$.

In fact, we have a commutative diagram of algebroids

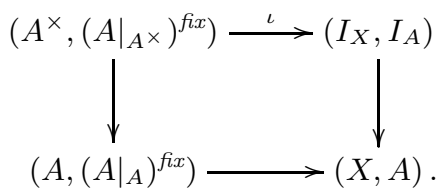

If $(X, A)$ is a strict algebroid, this is a diagram of strict algebroids.

Remark 1.58 (semi-simple algebroid inertia). There is also a semisimple version of the algebroid inertia. To define it, let $(X, A)$ be an algebroid, and consider the diagram of algebroids

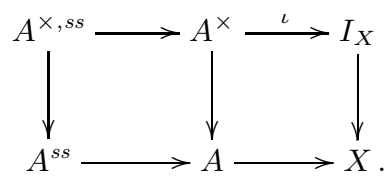

The square on the right was constructed in Remark 1.57 The morphism $A^{s s} \rightarrow A$ is the composition of a stratification and an open immersion (see Proposition 1.34), in particular it is inert. Hence we get an induced algebroid structure on $A^{s s}$. Requiring the square on the left to be a cartesian diagram of algebroids defines the algebraic stack $A^{\times, s s}$ and the algebroid structure over it.

If $(X, A)$ is a strict algebroid, all objects in Diagram (9) are strict, and $\iota$ is an isomorphism. We are then justified in defining $I_{X}^{s s}=A^{\times, s s}$, and calling it the semi-simple algebroid inertia of $X$. In the general case we define $I_{X}^{\circ, s s}=A^{\times, s s}$, and also call it the semi-simple algebroid inertia, by a slight abuse of language.

\section{Idempotents and algebroids}

Lemma 1.59. Let $a \in A^{\times, s s}$ be a semi-simple invertible global section of an algebra bundle $A \rightarrow X$. Let $\widetilde{A}^{\times, s s}=A^{\times, s s} / \mathbb{G}_{m}$ be the quotient of 
$A^{\times, s s}$ by the subgroup of scalars. Consider the action of the group $A^{\times}$on $A^{\times, s s} \rightarrow \widetilde{A}^{\times, s s}$ by conjugation. The induced group homomorphism

$$
\operatorname{Stab}_{A \times}(a) \longrightarrow \operatorname{Stab}_{A} \times[a]
$$

where $[a]$ is the class of a in $\widetilde{A}^{\times, s s}$, is an open immersion.

Proof. Let $Y_{a}$ be the relative spectrum of $\mathscr{O}_{X}[a]$ over $X$. The epimorphism of commutative algebras $\mathscr{O}_{X}[x] \rightarrow \mathscr{O}_{X}[a]$ gives rise to a closed immersion $\varphi: Y_{a} \rightarrow\left(\mathbb{G}_{m}\right)_{X}$, because $a$ is invertible. We get an induced proper morphism

$$
\begin{aligned}
Y_{a} \times_{X} Y_{a} & \longrightarrow\left(\mathbb{G}_{m}\right)_{X} \\
(\lambda, \mu) & \longmapsto \varphi(\lambda) / \varphi(\mu) .
\end{aligned}
$$

As $Y_{a} \rightarrow X$ is unramified, the diagonal $Y_{a} \rightarrow Y_{a} \times_{X} Y_{a}$ is an open immersion, so the complement, denoted $\left(Y_{a} \times Y_{a}\right)^{\neq}$, is closed in $Y_{a} \times Y_{a}$, and hence proper over $X$. Hence the image of $\left(Y_{a} \times Y_{a}\right)^{\neq}$in $\left(\mathbb{G}_{m}\right)_{X}$, denoted by $Z$, is closed. The complement of $Z$ in $\left(\mathbb{G}_{m}\right)_{X}$ is hence an open neighborhood $U$ of the identity section.

We have a cartesian diagram

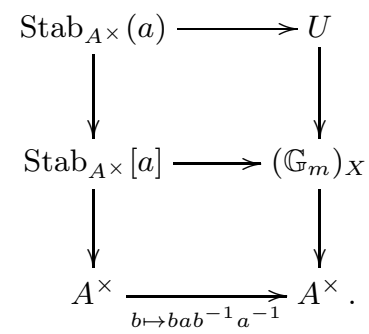

The lower square is cartesian by the definition of $\operatorname{Stab}_{A \times}[a]$. The fact that the upper square is cartesian follows from the fact that the image of $\operatorname{Stab}_{A} \times[a]$ in $\left(\mathbb{G}_{m}\right)_{X}$ is contained in the image of $[10)$. The latter claim follows from the fact that if ${ }^{b} a=\lambda a$, for a scalar $\lambda \in\left(\mathbb{G}_{m}\right)_{X}$, then $\lambda$ is necessarily a quotient of eigenvalues of $a$.

Remark 1.60. Let $(X, A)$ be an algebroid, and let $Y=E_{k}(A)$ be the stack of complete labelled sets of $k$ orthogonal idempotents in $A$. Let us write $B=\left(\left.A\right|_{Y}\right)^{e_{1}, \ldots, e_{k}}$, for the subalgebra of $\left.A\right|_{Y}$, consisting of elements commuting with each of the $k$ tautological idempotents in $\left.A\right|_{Y}$. The homomorphism $\iota: A^{\times} \rightarrow I_{X}$ restricts to a homomorphism $B^{\times} \rightarrow I_{Y}$, and defines an algebroid structure over $Y$. The algebra $B$ is endowed with a canonical complete set of orthogonal central idempotents, and hence decomposes as a product $B=B_{1} \times \ldots \times B_{k}$.

Let $(X, A)$ be an algebroid, where $A$ is an algebra bundle, and let $e_{1}, \ldots, e_{k}$ be a complete set of orthogonal central idempotents in $A$, decomposing $A$ into a product of algebra bundles $A=A_{1} \times \ldots \times A_{k}$. We get an induced decomposition

$$
A^{\times, s s}=A_{1}^{\times, s s} \times \ldots \times A_{k}^{\times, s s} \subset A,
$$


and the algebroid structure on $A^{\times, s s}$ is the fibered product over $X$ of the algebroid structures on the $A_{i}^{\times, s s}, i=1, \ldots, k$.

We obtain an embedding of algebras $\alpha: \mathbb{A}_{Y}^{k} \rightarrow A$. Via $\alpha$, the torus $\mathbb{G}_{m}^{k}$ acts on $A$ by left multiplication. The action of $\mathbb{G}_{m}^{k}$ on $A$ preserves the semi-simple units and the restricted action of $\mathbb{G}_{m}^{k}$ on $A^{\times, s s}$ is fiberwise free, so the quotient

$$
\widetilde{A}^{\times, s s}=A^{\times, s s} / \mathbb{G}_{m}^{k}=A_{1}^{\times, s s} / \mathbb{G}_{m} \times \ldots \times A_{k}^{\times, s s} / \mathbb{G}_{m}
$$

is representable over $X$.

Proposition 1.61. We claim that $\widetilde{A}^{\times, s s}$ has a canonical algebroid structure, and we get an induced commutative diagram

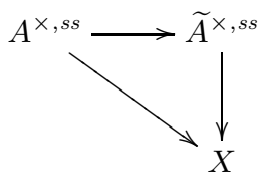

of algebroids, where the horizontal map is inert, hence a principal $\mathbb{G}_{m^{-}}^{k}$ bundle of algebroids.

Proof. Because inert morphisms of algebroids are stable under composition and pullback, this claim reduces to the case $k=1$, and $e_{1}=1$, which we will now consider.

Let us denote the tautological section of $A^{\times}$over $A^{\times, s s}$ by $a$. Then the algebra over $A^{\times, s s}$ is given by the centralizer algebra $\left(\left.A\right|_{A^{\times, s s}}\right)^{a}$. This algebra descends to the quotient $\widetilde{A}^{\times, s s}$, because the centralizer of an algebra element does not depend on its equivalence class. Let us denote this descended algebra by $\left(\left.A\right|_{\widetilde{A} \times, s s}\right)^{[a]}$. The units in this algebra are identified with

which is an open subgroup of

$$
\left(\left.A^{\times}\right|_{\widetilde{A} \times, s s}\right)^{[a]}=\left.\operatorname{Stab}_{\left(A^{\times}\right.}\right|_{\widetilde{A} \times, s s}(a),
$$

$$
\operatorname{Stab}_{\left(A \times\left.\right|_{\widetilde{A} \times, s s}\right)}[a],
$$

by Lemma 1.59 We also have a cartesian diagram

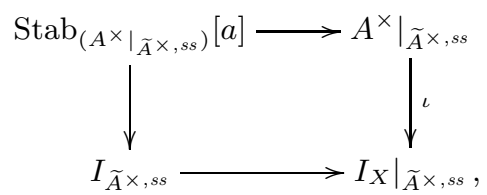

because the inertia stack $I_{\widetilde{A}^{\times, s s}}$ can be identified as

$$
I_{\widetilde{A}^{\times, s s}}=\left\{([a], \varphi) \in \widetilde{A}^{\times, s s} \times\left._{X} I_{X}\right|^{\varphi}[a]=[a]\right\} .
$$

This proves that $\operatorname{Stab}_{\left(\left.A^{\times}\right|_{\widetilde{A}^{\times}, s s}\right)}[a]$ is an open subgroup of $I_{\widetilde{A}^{\times}, s s}$. Composing our two open immersions, we obtain an open immersion of groups over $\widetilde{A}^{\times, s s}$ from $\left(\left.A^{\times}\right|_{\widetilde{A}^{\times, s s}}\right)^{[a]}$ to $I_{\widetilde{A}^{\times, s s}}$, endowing $\widetilde{A}^{\times, s s}$ with the structure of an algebroid, as required.

We get an induced morphism of algebroids $A^{\times, s s} \rightarrow \widetilde{A}^{\times, s s}$, which is inert, by construction. 
Remark 1.62. Even if $(X, A)$ is strict, $\widetilde{A}^{\times, s s}$ is not necessarily strict. It is this construction, in fact, which makes it impossible for us to restrict attention to strict algebroids.

Remark 1.63. Let $(\mathfrak{M}, \mathfrak{A})$ be the algebroid underlying a linear algebraic stack. Suppose that $\mathfrak{M}$ admits direct sums. Let $\mathfrak{M}_{*}$ be the complement of the zero object in $\mathfrak{M}$. We obtain a canonical morphism of algebroids

$$
\begin{aligned}
\underbrace{\mathfrak{M}_{*} \times \ldots \times \mathfrak{M}_{*}}_{n \text { times }} & \longrightarrow E_{n}(\mathfrak{A}) \\
& \left(x_{1}, \ldots, x_{n}\right) \\
\longmapsto & \left(x_{1} \oplus \ldots \oplus x_{n} ; \pi_{1} \ldots, \pi_{n}\right),
\end{aligned}
$$

where $\pi_{1}, \ldots, \pi_{n}$ are the projectors corresponding to the factors $x_{1}, \ldots, x_{n}$ of $x_{1} \oplus \ldots \oplus x_{n}$. Over every $R$-scheme $S$, this morphism is fully faithful. The underlying morphism of algebraic stacks (11) is a monomorphism.

If we require all fibers $\mathfrak{M}(S)$ to be Karoubian, i.e., we require all idempotents to admit the corresponding direct summands, (11) is an isomorphism of algebroids.

We call a linear algebraic stack $\mathfrak{M}$ Karoubian, if it admits direct sums and all fibers are Karoubian.

\section{Algebroid representations}

Definition 1.64. Let $X$ be an algebroid. A representation of $X$ is a morphism of algebroids $\rho: X \rightarrow \mathfrak{V e c t}$, to the algebroid underlying the linear stack $\mathfrak{V e c t}$ of vector bundles. If $\rho$ factors through vector bundles of rank $n$, i.e., defines a morphism $X \rightarrow B \mathrm{GL}_{n}$, with its natural algebroid structure (see Example 1.41), we say that $\rho$ has rank $n$.

If the algebroid morphism $X \rightarrow B \mathrm{GL}_{n}$ is representable, we call the representation $\rho$ faithful.

To give a representation of the algebroid $(X, A)$ is equivalent to specifying a vector bundle $V$ over $X$, together with a morphism of algebras $A \rightarrow \operatorname{End}(V)$, such that the induced morphism on unit groups $A^{\times} \rightarrow G L(V)$ makes the diagram

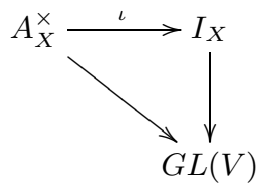

commute, where $I_{X} \rightarrow G L(V)$ is the inertia representation given by the vector bundle $V / X$.

The representation $V$ of rank $n$ is faithful if and only if $A \rightarrow \operatorname{End}(V)$ is a monomorphism of algebras over $X$. If this is the case, the underlying morphism of stacks $X \rightarrow B \mathrm{GL}_{n}$ is Deligne-Mumford representable, and the $\mathrm{GL}_{n}$-bundle of frames $Y=\underline{\operatorname{Isom}}\left(V, \mathscr{O}^{n}\right)$ is represented by a DeligneMumford stack. 
So a faithful representation identifies $X$ as a quotient stack $X=$ $\mathrm{GL}_{n} \backslash Y$, where $Y$ is a Deligne-Mumford stack. The algebroid structure on $X$ is then given by a $\mathrm{GL}_{n}$-invariant subalgebra $B \hookrightarrow M_{n \times n} \times Y$, such that the subgroup $B^{\times} \hookrightarrow \mathrm{GL}_{n} \times Y$ is equal to the subgroup $\operatorname{Stab}_{\mathrm{GL}_{n}} Y \hookrightarrow$ $\mathrm{GL}_{n} \times Y$.

Remark 1.65. Every algebroid $(X, A)$, with $A$ an algebra bundle, admits the tautological adjoint representation, given by the adjoint representation of the algebra $A$ on itself. By contrast, the representation of $A$ on itself by left multiplication is not an algebroid representation, unless $A=0$.

Proposition 1.66. Every algebroid $(X, A)$ admits a stratification $X^{\prime} \subset$ $X$, such that the restricted algebroid $\left(X^{\prime},\left.A\right|_{X^{\prime}}\right)$ admits a faithful representation.

Proof. Without loss of generality, assume that $A$ is an algebra bundle with smooth unit group $A^{\times}$, and that $X$ is a connected isotrivial gerbe over the Deligne-Mumford stack $S$. Thus $X \rightarrow S$ admits a section over a finite étale cover $S^{\prime} \rightarrow S$.

To begin with, we consider the case where $S^{\prime}=S$, i.e., the case where $X$ is a trivial gerbe over $S$. By Remark 1.43 we can assume that $A$ descends to $S$, and that we are dealing with the algebroid $\left(B A^{\times}, A^{\times} \backslash A\right)$. Then we can consider the representation of $A$ on itself by left multiplication $\ell: A \rightarrow \operatorname{End}(A)$. It restricts to a representation of $S$-group schemes $\ell: A^{\times} \rightarrow G L(A)$. We get an induced morphism of algebraic stacks $B A^{\times} \rightarrow B G L(A)$, which is covered by the morphism of algebras $A^{\times} \backslash A \rightarrow B G L(A) \backslash \operatorname{End}(A)$. Since $\ell: A \rightarrow \operatorname{End}(A)$ is injective, this gives the required faithful representation of $\left(B A^{\times}, A^{\times} \backslash A\right)$.

(Note that this construction does not contradict Remark 1.65. The vector bundle over $X$ defined by the left representation of $A^{\times}$on itself is different from the vector bundle underlying the algebroid $A$ over $X$, which is given by the adjoint representation of $A^{\times}$on itself.)

Now consider the general case. The pullback $\left(X^{\prime}, A^{\prime}\right)$ of $(X, A)$ to $S^{\prime}$ is again an algebroid, as $X^{\prime} \rightarrow X$ is inert. Since $X^{\prime} \rightarrow S^{\prime}$ is a trivial gerbe, $\left(X^{\prime}, A^{\prime}\right)$ admits a faithful representation $A^{\prime} \rightarrow \operatorname{End}(V)$, on a vector bundle $V$ over $X^{\prime}$. Let $\pi: X^{\prime} \rightarrow X$ be the projection. Then $\pi_{*} V$ is a faithful representation of $A$. In fact, by adjunction, the embedding $\pi^{*} A \rightarrow$ $\operatorname{End}(V)$ gives rise to an embedding $A \rightarrow \pi_{*} \operatorname{End}(V) \rightarrow \operatorname{End}\left(\pi_{*} V\right)$.

\section{Clear algebroids}

Suppose that $(X, A)$ is an algebroid, and that $X$ is a connected gerbe over the Deligne-Mumford stack $\bar{X}$. Then the centre $Z(A)$ descends to a commutative finite type algebra over $\bar{X}$, by Lemma 1.20

Definition 1.67. We call an algebroid $(X, A)$ clear, if

(i) $A$ and $Z(A)$ are algebra bundles over $X$,

(ii) $X$ is a connected isotrivial gerbe over $\bar{X}$, 
(iii) the Deligne-Mumford stack $\bar{X}$ is connected,

(iv) $Z E(A) \rightarrow X$ is finite étale.

For a clear algebroid, $Z E(A)$ and $P Z E(A)$ descend to finite étale $\bar{X}$ schemes. The definitions of central rank, split central rank, and central type apply to clear algebroids.

For every algebroid $(X, A)$, over a finite type algebraic stack $X$, there exists a stratification of $X$, such that the restricted algebroids over the pieces of the stratification are all clear. This follows from Proposition 1.5 and Lemma 1.26 . 


\section{The spectrum of semi-simple inertia}

Let $K(\mathrm{DM})$ be the $\mathbb{Q}$-vector space on (isomorphism classes of) finite type Deligne-Mumford stacks, modulo scissor relations and bundle relations, i.e., equations of the form $[Y]=[F \times X]$, whenever $Y \rightarrow X$ is a fibre bundle with special structure group and fibre $F$. The product over $\operatorname{Spec} R$ makes $K(\mathrm{DM})$ a $\mathbb{Q}$-algebra. We write $q$ for the class of the affine line in $K(\mathrm{DM})$.

Let $\mathfrak{M}$ be a linear algebraic stack, and $\mathfrak{A} \rightarrow \mathfrak{M}$ its universal endomorphism algebra. Recall that $(\mathfrak{M}, \mathfrak{A})$ is an algebroid (c.f. Example 1.40).

\section{Stack functions}

Definition 2.1. A stack function is a representable morphism of algebroids $(X, A) \rightarrow(\mathfrak{M}, \mathfrak{A})$, such that $X$ is of finite type.

The $K$-module of $\mathfrak{M}$, notation $K(\mathfrak{M})$, is the free $\mathbb{Q}$-vector space on (isomorphism classes of) stack functions, modulo the scissor and bundle relations relative to $(\mathfrak{M}, \mathfrak{A})$. The class in $K(\mathfrak{M})$ defined by a stack function $X \rightarrow \mathfrak{M}$ will be denoted $[X \rightarrow \mathfrak{M}]$.

A scissor relation relative $\mathfrak{M}$ is

$$
[X \rightarrow \mathfrak{M}]=[Z \rightarrow X \rightarrow \mathfrak{M}]+[X \backslash Z \rightarrow X \rightarrow \mathfrak{M}],
$$

for any closed immersion of algebroids $Z \hookrightarrow X$, and any stack function $X \rightarrow \mathfrak{M}$. The substacks $Z$ and $X \backslash Z$ are endowed with their respective pullback algebroids.

A bundle relation relative $\mathfrak{M}$ is

$$
[Y \rightarrow X \rightarrow \mathfrak{M}]=[F \times X \rightarrow X \rightarrow \mathfrak{M}]
$$

for any fibre bundle $Y \rightarrow X$ of algebroids with special structure group and fibre $F$, see Definition 1.53 .

There is an action of $K(\mathrm{DM})$ on $K(\mathfrak{M})$, given by

$$
[Z] \cdot[X \rightarrow \mathfrak{M}]=[Z \times X \rightarrow X \rightarrow \mathfrak{M}] .
$$

This action makes $K(\mathfrak{M})$ into a $K(\mathrm{DM})$-module.

The additive zero in $K(\mathfrak{M})$ is given by the empty algebroid

$$
0=[\varnothing \rightarrow \mathfrak{M}] .
$$

If $\mathfrak{M}$ admits a zero object (Definition 1.16), we denote the corresponding stack function by $1=[\operatorname{Spec} R \stackrel{0}{\longrightarrow} \mathfrak{M}]$. We can use it to embed $K(\mathrm{DM})$ into $K(\mathfrak{M})$ via $[X] \mapsto[X] \cdot 1=[X \rightarrow \operatorname{Spec} R \stackrel{0}{\longrightarrow} \mathfrak{M}]$. We will always assume that $\mathfrak{M}$ admits a zero object. 


\section{The filtration by split central rank}

We call a stack function $X \rightarrow \mathfrak{M}$ clear, if $X$ is a clear algebroid (Definition 1.67).

Definition 2.2. We introduce the filtration by split central rank $K^{\geq k}(\mathfrak{M})$ on $K(\mathfrak{M})$, by declaring $K^{\geq k}(\mathfrak{M})$ to be generated as a $\mathbb{Q}$-vector space by clear stack functions $[X \rightarrow \mathfrak{M}]$, such that $A_{X}$ admits $k$ orthogonal central non-zero idempotents (globally).

Alternatively, $K^{\geq k}(\mathfrak{M})$ is generated by $[X \rightarrow \mathfrak{M}]$, where $X$ is a clear algebroid such that $P Z E\left(A_{X}\right)$ has at least $k$ components.

Each filtered piece $K^{\geq k}(\mathfrak{M})$ is stable under scalar multiplication by $K(\mathrm{DM})$. Let us introduce the abbreviation

$$
K^{\geq k} / K^{>k}(\mathfrak{M})=K^{\geq k}(\mathfrak{M}) / K^{>k}(\mathfrak{M}) .
$$

Remark 2.3. Trying to define a direct sum decomposition of $K(\mathfrak{M})$ by split central rank would not work, because a clear algebroid $X$ of split central rank $k$ may very well admit a closed substack $Z \subset X$ whose restricted algebroid is again clear, but of split central rank larger than $k$. Similarly, the bundle relations do not respect split central rank.

The zero ring has no non-zero central idempotents, but any non-zero ring has at least one. Therefore, $K(\mathrm{DM}) \subset K(\mathfrak{M})$ is a complement for $K^{>0}(\mathfrak{M})$ in $K(\mathfrak{M})=K^{\geq 0}(\mathfrak{M})$, i.e., $K(\mathfrak{M})=K(\mathrm{DM}) \oplus K^{>0}(\mathfrak{M})$. In particular, we have

$$
K^{\geq 0}(\mathfrak{M}) / K^{>0}(\mathfrak{M})=K(\mathrm{DM})
$$

\subsection{The idempotent operators $E_{r}$}

Let $E_{r}$ denote the operator on $K(\mathfrak{M})$ which maps a stack function $[X \rightarrow$ $\mathfrak{M}]$ to $\left[E_{r}(X) \rightarrow X \rightarrow \mathfrak{M}\right]$, where $E_{r}(X)=E_{r}\left(A_{X}\right)$ is the stack of $r$ tuples of non-zero orthogonal idempotents adding to unity in $A_{X}$, see Definition 1.37 The algebroid structure on $E_{r}(X)$ is described in Remark 1.60

The operators $E_{r}$ are well-defined, because applying $E_{r}$ to a stratification or a fiber bundle of algebroids gives rise to another inert morphism of algebroids of the same type. The operators $E_{r}: K(\mathfrak{M}) \rightarrow K(\mathfrak{M})$ are $K(\mathrm{DM})$-linear.

This definition applies also to $r=0$. The stack $E_{0}(X)$ is empty if $A_{X} \neq 0$, and $E_{0}(X)=X$, if $X$ is a Deligne-Mumford stack. Hence $E_{0}$ is diagonalizable, and has eigenvalues 0 and 1 . The kernel (0-eigenspaces) is $K^{>0}(\mathfrak{M}) \subset K(\mathfrak{M})$, the image (1-eigenspaces) is denoted by $K^{0}(\mathfrak{M}) \subset$ $K(\mathfrak{M})$, and is generated by all stack functions $[X \rightarrow \mathfrak{M}]$, where $X$ is a Deligne-Mumford stack. In fact, $K^{0}(\mathfrak{M})=K(\mathrm{DM}) \subset K(\mathfrak{M})$.

For $r=1$, the operator $E_{1}$ vanishes on stack functions $[X \rightarrow \mathfrak{M}]$, where $X$ is a Deligne-Mumford stack, and acts as identity on stack functions for which $A_{X} \neq 0$. Hence, $E_{1}$ is also diagonalizable with eigenvalues 0 
and 1. The kernel of $E_{1}$ is $K^{0}(\mathfrak{M})$, and the image is $K^{>0}(\mathfrak{M})$. Hence $E_{0}$ and $E_{1}$ are complementary idempotent operators on $K(\mathfrak{M})$, i.e., they are orthogonal to each other and add up to the identity.

Recall the Stirling number of the second kind, $S(k, r)$, which is defined in such a way that $r ! S(k, r)$ is the number of surjections from $\underline{k}$ to $\underline{r}$. Here, and elsewhere, We write $\underline{n}=\{1, \ldots, n\}$.

Theorem 2.4. The operators $E_{r}$, for all $r \geq 0$, preserve the filtration $K^{\geq k}(\mathfrak{M})$ by split central rank. On the subquotient $K^{\geq k} / K^{>k}(\mathfrak{M})$, the operator $E_{r}$ acts as multiplication by $r ! S(k, r)$.

Proof. Consider a clear algebroid $(X, A)$ with a morphism $X \rightarrow \mathfrak{M}$ defining the stack function $[X \rightarrow \mathfrak{M}]$ in $K(\mathfrak{M})$. Let $n$ be the central rank of $X$, and $k$ the split central rank of $X$. The filtered piece $K^{\geq k}(\mathfrak{M})$ is generated by such $[X \rightarrow \mathfrak{M}]$.

Denote by $X \rightarrow \bar{X}$ the coarse Deligne-Mumford stack of $X$. By assumption, both $X$ and $\widetilde{X}$ are connected and hence admit Grothendieck style Galois theories (see [16, Section 4]).

Let $\tilde{X} \rightarrow X$ be a connected Galois cover with Galois group $\Gamma$, which trivializes $P Z E(A) \rightarrow X$. As $P Z E(A)$ descends to $\bar{X}$, this Galois cover can be constructed as a pullback from $\bar{X}$. Therefore, the morphism $\widetilde{X} \rightarrow$ $X$ is inert and hence $\widetilde{X}$ inherits, via pullback, the structure of an algebroid, and hence $[\widetilde{X} \rightarrow X \rightarrow \mathfrak{M}]$ is a stack function.

Recall that the degree of the cover $P Z E(A) \rightarrow X$ is $n$, and the number of components of $P Z E(A)$ is $k$.

By labelling the components of the pullback of $P Z E(A)$ to $\tilde{X}$, we obtain an action of $\Gamma$ on the set $\underline{n}=\{1, \ldots, n\}$ and an isomorphism of finite étale covers of $X$

$$
\begin{aligned}
& \tilde{X} \times_{\Gamma} \underline{n} \stackrel{\simeq}{ } \operatorname{PZE}(A) \\
& {[x, \nu] } \longmapsto e_{[x, \nu]} .
\end{aligned}
$$

Both source and target of this isomorphism support natural algebroids and the isomorphism preserves them. The number of orbits of $\Gamma$ on $\underline{n}$ is $k$.

Then we also have an isomorphism

$$
\begin{aligned}
\widetilde{X} \times_{\Gamma} \operatorname{Epi}(\underline{n}, \underline{r}) \stackrel{\simeq}{\longrightarrow} Z E_{r}(A) \\
{[x, \varphi] \longmapsto\left(\sum_{\varphi(\nu)=\rho} e_{[x, \nu]}\right)_{\rho=1, \ldots, r}, }
\end{aligned}
$$

where $Z E_{r}$ denotes the stack of labelled complete sets of $r$ orthogonal central idempotents. Again, both stacks involved are in fact algebroids, and this isomorphism is an isomorphism of algebroids.

Hence, we may calculate as follows (all stacks involved are endowed 
with their natural algebroid structures):

$$
\begin{aligned}
Z E_{r}[X \rightarrow \mathfrak{M}] & =\left[\tilde{X} \times_{\Gamma} \operatorname{Epi}(\underline{n}, \underline{r}) \rightarrow \mathfrak{M}\right] \\
& =\left[\tilde{X} \times_{\Gamma} \coprod_{\varphi \in \operatorname{Epi}(\underline{n}, \underline{r}) / \Gamma} \Gamma / \operatorname{Stab}_{\Gamma} \varphi \rightarrow \mathfrak{M}\right] \\
& =\sum_{\varphi \in \operatorname{Epi}(\underline{n}, \underline{r}) / \Gamma}\left[\tilde{X} / \operatorname{Stab}_{\Gamma} \varphi \rightarrow \mathfrak{M}\right] \\
& =\sum_{\varphi \in \operatorname{Epi}(\underline{n}, \underline{r})^{\Gamma}}[X \rightarrow \mathfrak{M}]+\sum_{\substack{\varphi \in \operatorname{Epi}(\underline{n}, \underline{r}) / \Gamma \\
\operatorname{Stab}_{\Gamma} \not \neq \Gamma}}\left[\tilde{X} / \operatorname{Stab}_{\Gamma} \varphi \rightarrow \mathfrak{M}\right]
\end{aligned}
$$

Now, we have $\operatorname{Epi}(\underline{n}, \underline{r})^{\Gamma}=\operatorname{Epi}(\underline{n} / \Gamma, \underline{r})$, and hence

$$
\# \operatorname{Epi}(\underline{n}, \underline{r})^{\Gamma}=r ! S(k, r) \text {. }
$$

Thus, we conclude,

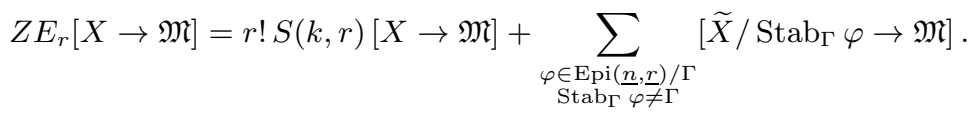

For any proper subgroup $\Gamma^{\prime} \subset \Gamma$, the quotient $X^{\prime}=\widetilde{X} / \Gamma^{\prime}$ is an intermediate cover $\widetilde{X} \rightarrow X^{\prime} \rightarrow X$, such that $X^{\prime} \neq X$. The pullback of $P Z E(A)$ to $X^{\prime}$ has more than $k$ components, because the number of orbits of $\Gamma^{\prime}$ on $\underline{n}$ is larger than $k$. Thus we have proved the theorem for $Z E_{r}$, instead of $\bar{E}_{r}$.

Now observe that $Z E_{r}(A) \subset E_{r}(A)$ is a closed substack, because $Z E_{r}(A) \rightarrow X$ is proper and $E_{r}(A) \rightarrow X$ is separated. So we can write

$$
E_{r}[X \rightarrow \mathfrak{M}]=Z E_{r}[X \rightarrow \mathfrak{M}]+\left[N Z E_{r}(A) \rightarrow X \rightarrow \mathfrak{M}\right]
$$

where $N Z E_{r}(A)$ is the complement of $Z E_{r}(A)$ in $E_{r}(A)$. To prove that $\left[N Z E_{r}(A) \rightarrow \mathfrak{M}\right] \in K^{>k}(\mathfrak{M})$, let $Y \hookrightarrow N Z E_{r}(A)$ be a locally closed embedding, such that the algebroid $\left.\left(E_{r}(A), A^{f i x}\right)\right|_{Y}$ is clear.

Consider the embedding of algebras $\left.\left.A^{f i x}\right|_{Y} \hookrightarrow A\right|_{Y}$. It induces an embedding of commutative algebras $Z\left(\left.A\right|_{Y}\right) \hookrightarrow Z\left(\left.A^{f i x}\right|_{Y}\right)$, because $Z\left(\left.A\right|_{Y}\right) \subset$ $\left.A^{f i x}\right|_{Y}$. The algebra $\left.A\right|_{Y}$ comes with $r$ tautological idempotent sections, all of which are contained in $Z\left(\left.A^{f i x}\right|_{Y}\right)$, but at least one of which is not contained in $Z\left(\left.A\right|_{Y}\right)$. So by Proposition [1.36 (ii), the split central rank of $\left.A^{f i x}\right|_{Y}$ is strictly larger than the split central rank of $\left.A\right|_{Y}$. The latter is at least as big as $k$, the split central rank of $A$, because the split central rank cannot decrease under base extension. This shows that $[Y \rightarrow \mathfrak{M}] \in K^{>k}(\mathfrak{M})$ and finishes the proof.

Corollary 2.5. The operators $E_{r}$, for $r \geq 0$ are simultaneously diagonalizable. The common eigenspaces form a family $K^{k}(\mathfrak{M})$ of subspaces of $K(\mathfrak{M})$ indexed by non-negative integers $k \geq 0$, and

$$
K(\mathfrak{M})=\bigoplus_{k \geq 0} K^{k}(\mathfrak{M})
$$


Moreover, for every $r \geq 0$,

$$
K^{\geq r}(\mathfrak{M})=\bigoplus_{k \geq r} K^{k}(\mathfrak{M})
$$

Let $\pi_{k}$ denote the projection onto $K^{k}(\mathfrak{M})$. We have

$$
E_{r} \pi_{k}=r ! S(k, r) \pi_{k}
$$

for all $r \geq 0, k \geq 0$.

Proof. First remark that for given $r$, the numbers $r ! S(k, r)$ form a monotone increasing sequence of integers.

Then note that the operators $E_{r}$ pairwise commute: the composition $E_{r} \circ E_{r^{\prime}}$ associates to an algebroid $(X, A)$ the stack of pairs $\left(e, e^{\prime}\right)$, where both $e$ and $e^{\prime}$ are complete families of non-zero orthogonal idempotents in $A$, the length of $e$ being $r$ and the length of $e^{\prime}$ being $r^{\prime}$, and the members of $e$ commuting with the members of $e^{\prime}$.

Finally, let us prove that, for every $k$ and every $r$, the $\mathbb{Q}$-vector space $K^{\geq k}(\mathfrak{M})$ is a union of finite-dimensional subspaces invariant by $E_{r}$.

For this, define $K(\mathfrak{M})_{\leq N}$ to be generated as $\mathbb{Q}$-vector space by stack functions $[X \rightarrow \mathfrak{M}]$, where $X$ is a clear algebroid, such that the rank of the vector bundle underlying the algebra $A_{X} \rightarrow X$ is bounded above by $N$. This is an ascending filtration of $K(\mathfrak{M})$, which is preserved by $E_{r}$. Set

$$
K^{\geq k}(\mathfrak{M}) \cap K(\mathfrak{M})_{\leq N}=K^{\geq k}(\mathfrak{M})_{\leq N} .
$$

Suppose $x=[X \rightarrow \mathfrak{M}]$ is a stack function with $X$ a clear algebroid of split central rank $k$, and let $N$ be the rank of the vector bundle underlying $A_{X}$. Note that $k \leq N$, because for a commutative algebra, the number of primitive idempotents is bounded by the rank of the underlying vector bundle. We deduce that for $k>N$, we have $K^{\geq k}(\mathfrak{M})_{\leq N}=0$.

On the other hand, Theorem 2.4 implies by induction that

$$
E_{r}^{i}(x) \in \mathbb{Q} x+\mathbb{Q} E_{r}(x)+\ldots+\mathbb{Q} E_{r}^{i-1}(x)+K^{\geq k+i}(\mathfrak{M}) .
$$

Applying this for $i=N-k+1$, we see that

$$
E_{r}\left(E_{r}^{N-k}(x)\right) \in \mathbb{Q} x+\mathbb{Q} E_{r}(x)+\ldots+\mathbb{Q} E_{r}^{N-k}(x),
$$

and hence that $\mathbb{Q} x+\mathbb{Q} E_{r}(x)+\ldots+\mathbb{Q} E_{r}^{N-k}(x)$ is invariant under $E_{r}$.

This proves that any $x \in K^{\geq k}(\mathfrak{M})$ is contained in a finite-dimensional subspace invariant under $E_{r}$. Standard techniques from finite-dimensional linear algebra over $\mathbb{Q}$ now imply the result.

Remark 2.6. The proof of Theorem 2.4 and its corollary show that the central versions $Z E_{r}$ of the $E_{r}$ are also diagonalizable. On the other hand, the $Z E_{r}$ do not commute with each other, and so are less useful. 
Corollary 2.7. For $r \geq 1$, we have

$$
\operatorname{ker} E_{r}=\bigoplus_{k<r} K^{k}(\mathfrak{M}) .
$$

In particular, for any $x \in K(\mathfrak{M})$, we have $E^{r} x=0$, for $r \gg 0$.

Corollary 2.8. For every $k \geq 0$, we have

$$
\pi_{k}=\sum_{r=k}^{\infty} \frac{s(r, k)}{r !} E_{r},
$$

where the $s(n, k)$ are the Stirling numbers of the first kind. In particular, $\pi_{0}=E_{0}$, and

$$
\pi_{1}=\sum_{r=1}^{\infty} \frac{(-1)^{r+1}}{r} E_{r} .
$$

Proof. We have

$$
\mathrm{id}=\sum_{\ell \geq 0} \pi_{\ell},
$$

and hence

$$
E_{r}=\sum_{\ell \geq 0} E_{r} \pi_{\ell}=\sum_{\ell \geq 0} r ! S(\ell, r) \pi_{\ell},
$$

and therefore

$$
\begin{aligned}
\sum_{r \geq 0} \frac{s(r, k)}{r !} E_{r}=\sum_{r \geq 0} & \frac{s(r, k)}{r !} \sum_{\ell \geq 0} r ! S(\ell, r) \pi_{\ell} \\
& =\sum_{\ell \geq 0}\left(\sum_{r \geq 0} S(\ell, r) s(r, k)\right) \pi_{\ell}=\sum_{\ell \geq 0} \delta_{\ell, k} \pi_{\ell}=\pi_{k},
\end{aligned}
$$

by the inverse relationship between the Stirling numbers of the first and second kind.

Remark 2.9. The Stirling numbers of the first kind appear in the Taylor expansions of the powers of the logarithm:

$$
\sum_{r=k}^{\infty} \frac{s(r, k)}{r !} t^{r}=\frac{1}{k !} \log (1+t)^{k} .
$$

Definition 2.10. Let $t$ be a formal variable. We define the operator

$$
\pi_{t}: K(\mathfrak{M})[t] \longrightarrow K(\mathfrak{M})[t]
$$

by the formula

$$
\pi_{t}(\xi)=\sum_{k} \pi_{k}(\xi) t^{k}
$$

and extending $K(\operatorname{Var})[t]$-linearly. We can write, formally,

$$
\pi_{t}=\sum_{k} \pi_{k} t^{k} .
$$


Remark 2.11. We have the following convenient formula:

$$
\pi_{t}=\sum_{n}\left(\begin{array}{l}
t \\
n
\end{array}\right) E_{n} .
$$

It follows from Corollary 2.8, using the identity

$$
\sum_{k} \frac{s(n, k)}{n !} t^{k}=\left(\begin{array}{l}
t \\
n
\end{array}\right) .
$$

Example 2.12. The universal rank 2 vector bundle $G L_{2} \backslash \mathbb{A}^{2} \rightarrow B G L_{2}$, and its classifying morphism to $\mathfrak{V e c t}$ define a Hall algebra element $\left[B G L_{2} \rightarrow\right.$ $\mathfrak{V} \mathfrak{e c t}] \in K(\mathfrak{V} \mathfrak{e} \mathfrak{t})$, which we will abbreviate to $\left[B G L_{2}\right]$. To decompose $\left[B G L_{2}\right]$ into its pieces according to (12), we consider the action of $E_{2}$, as we have $E_{r}\left[B G L_{2}\right]=0$, for all $r>2$. In fact,

$$
E_{2}\left[B G L_{2}\right]=[B T], \quad \text { and } \quad E_{2}[B T]=2[B T],
$$

where $T$ is a maximal torus in $G L_{2}$. Thus $\mathbb{Q}\left[B G L_{2}\right]+\mathbb{Q}[B T]$ is a subspace of $K(\mathfrak{V e c t})$ invariant under $E_{2}$, and the matrix of $E_{2}$ acting on this subspace is

$$
\left(\begin{array}{ll}
0 & 0 \\
1 & 2
\end{array}\right)
$$

This matrix is lower triangular, with different numbers on the diagonal, hence diagonalizable over $\mathbb{Q}$. In fact, the diagonal entries are $2 S(1,2)=0$ and $2 S(2,2)=2$. Diagonalizing (13) gives the eigenvectors

(i) $v_{1}=\left[B G L_{2}\right]-\frac{1}{2}[B T]$ with eigenvalue 0 ,

(ii) $v_{2}=\frac{1}{2}[B T]$ with eigenvalue 2 .

Therefore, we have $v_{1} \in K^{1}(\mathfrak{V} \mathfrak{e c t})$ and $v_{2} \in K^{2}(\mathfrak{V} \mathfrak{e c t})$, and since $\left[B G L_{2}\right]=$ $v_{1}+v_{2}$, we have found the required decomposition of $\left[B G L_{2}\right]$.

\subsection{The spectrum of semisimple Inertia}

The connected semi-simple inertia operator on $K(\mathfrak{M})$ is the $\mathbb{Q}$-linear endomorphism

$$
\begin{aligned}
I^{\circ, s s}: K(\mathfrak{M}) & \longrightarrow K(\mathfrak{M}) \\
{[X \rightarrow \mathfrak{M}] } & \longmapsto\left[I_{X}^{\circ, s s} \rightarrow X \rightarrow \mathfrak{M}\right] .
\end{aligned}
$$

Here $I_{X}^{\circ, s s}=A_{X}^{\times, s s}$ denotes the semisimple algebroid inertia of the algebroid $X$, see Remark 1.58 . Note that $I^{\circ, s s}$ respects the scissor and bundle relations defining $\widetilde{K}(\mathfrak{M})$, and is linear over $K(\mathrm{DM})$, because passing to connected inertia commutes with inert pullbacks.

Note that $I^{\circ, s s}$ commutes with $E_{r}$, for every $r$. Both compositions $E_{r} \circ I^{\circ, s s}$ and $I^{\circ, s s} \circ E_{r}$ associate to an algebroid $(X, A)$ the stack of pairs $(a, e)$, where $a$ is a semi-simple unit in $A$, and $e$ a labelled complete set of $r$ orthogonal idempotents in $A$, all commuting with $a$. In particular, $I^{\circ, s s}$ preserves the filtration of $K(\mathfrak{M})$ by split central rank.

The composition $I^{\circ, s s} \circ E_{r}$ is divisible by $(q-1)^{r}$ : 
Proposition 2.13. For every $k \geq 0$, there exists a $K(\mathrm{DM})$-linear operator

$$
\widetilde{I}_{k}^{o, s s}: K(\mathfrak{M}) \longrightarrow K(\mathfrak{M}),
$$

such that

$$
I^{\circ, s s} \circ E_{k}=(q-1)^{k} \widetilde{I}_{k}^{\circ, s s} .
$$

Proof. Let $X$ be an algebroid. The algebra $A_{E_{k} X}$ is endowed with a tautological complete set of orthogonal central idempotents, and so we can apply the construction of Proposition 1.61 to obtain a principal $\mathbb{G}_{m^{-}}^{k}$ bundle of algebroids

$$
A_{E_{k} X}^{\times, s s} \longrightarrow \widetilde{A}_{E_{k} X}^{\times, s s}
$$

The assignment

$$
[X] \longmapsto\left[\widetilde{A}_{E_{k} X}^{\times, s s}\right]
$$

extends to a well-defined $K(\mathrm{DM})$-linear operator $K(\mathfrak{M}) \rightarrow K(\mathfrak{M})$, which we shall denote by $\widetilde{I}_{k}^{o, s s}$.

We record that for an algebroid $X$, the stack $\widetilde{I}_{k}^{s s} X$ is the stack of $(k+2)$ tuples $\left(x, e_{1}, \ldots, e_{k},[a]\right)$, where $x$ is an object of $X$, and $e_{1}, \ldots, e_{k}$ form a complete set of orthogonal idempotents in $A_{x}$, and $[a]$ is an equivalence class of semi-simple units in $A_{x}^{e_{1}, \ldots, e_{r}}$, where $a \sim \sum_{i=1}^{r} \lambda_{i} e_{i} a$, for $\lambda_{i} \in$ $\left.\mathfrak{O}_{X}\right|_{x}$.

The equation

$$
I^{\circ, s s} \circ E_{k}=(q-1)^{k} \widetilde{I}_{k}^{\circ, s s},
$$

follows from the fact that

$$
\left[A_{E_{k} X}^{\times, s s}\right]=(q-1)^{k}\left[\widetilde{A}_{E_{k} X}^{\times, s s}\right]
$$

which holds because of the bundle relations in $K(\mathfrak{M})$.

Corollary 2.14. The map which $I^{\circ, s s}$ induces on the subquotient $K^{\geq k} / K^{>k}(\mathfrak{M})$ is divisible by $(q-1)^{k}$ :

$$
\left.I^{\circ, s s}\right|_{K \geq k / K>k(\mathfrak{M})}=\left.\frac{1}{k !}(q-1)^{k} \widetilde{I}_{k}^{\mathrm{o}, s s}\right|_{K \geq k / K>k(\mathfrak{M})} .
$$

Proof. This is because on $K^{\geq k} / K^{>k}(\mathfrak{M})$, the operator $E_{k}$ acts as multiplication by $k$ !.

We will use as scalars the localization of $\mathbb{Q}[q]$ at the maximal ideal $(q-1)$, denoted $\mathbb{Q}[q]_{(q-1)}$, thus inverting all rational polynomials in $q$, which do not vanish at $q=1$. We extend scalars on $K(\mathfrak{M})$ as well, and consider

$$
K(\mathfrak{M})_{(q-1)}=\mathbb{Q}[q]_{(q-1)} \cdot K(\mathfrak{M}) \subset K(\mathfrak{M})(q)=K(\mathfrak{M}) \otimes_{\mathbb{Q}[q]} \mathbb{Q}(q) .
$$

Note that this definition ensures that $K(\mathfrak{M})_{(q-1)}$ is $(q-1)$-torsion free. The direct sum decomposition (12) extends to $K(\mathfrak{M})_{(q-1)}$, and the operator $I^{\circ, s s}$ extends to a $\mathbb{Q}[q]_{(q-1)}$-linear operator

$$
I^{\circ, s s}: K(\mathfrak{M})_{(q-1)} \longrightarrow K(\mathfrak{M})_{(q-1)} \text {. }
$$


For a partition $\lambda \vdash n$, we define

$$
\mathscr{Q}_{\lambda}=\prod_{i \in \lambda}\left(q^{i}-1\right) .
$$

This is a polynomial in $q$, of degree $n$, which vanishes to order $|\lambda|$ at $q=1$. We also define

$$
\widetilde{\mathscr{Q}}_{\lambda}=k ! \prod_{i \in \lambda} \frac{q^{i}-1}{q-1} .
$$

This is a polynomial in $q$, which is invertible in $\mathbb{Q}[q]_{(q-1)}$.

Theorem 2.15. The operator

$$
\widetilde{I}_{k}^{\circ, s s}: K^{\geq k} / K^{>k}(\mathfrak{M})_{(q-1)} \longrightarrow K^{\geq k} / K^{>k}(\mathfrak{M})_{(q-1)}
$$

is diagonalizable. Its eigenvalue spectrum consists of all $\widetilde{\mathscr{Q}}_{\lambda}$, for partitions $\lambda$ of length $|\lambda|=k$.

Proof. We will fix $k$, and work throughout in the subquotient $K^{\geq k} / K^{>k}(\mathfrak{M})_{(q-1)}$, restricting all operators tacitly to this subquotient. Note that, as a $\mathbb{Q}[q]_{(q-1)}$-module, $K^{\geq k} / K^{>k}(\mathfrak{M})_{(q-1)}$ is isomorphic to $K^{k}(\mathfrak{M})_{(q-1)}$, and is hence $(q-1)$-torsion free.

We order partitions of length $k$ by divisibility. If $\lambda$ and $\mu$ are partitions with $|\lambda|=k$ and $|\mu|=k$, we write $\lambda \mid \mu$, if there exists a permutation $\sigma$ of $\underline{k}$, such that $\lambda_{i} \mid \mu_{\sigma(i)}$, for all $i=1, \ldots, k$. This is a partial ordering on the partitions of length $k$. We write

$$
K^{\geq \lambda}(\mathfrak{M})_{(q-1)}
$$

for the $\mathbb{Q}[q]_{(q-1)}$-subspace generated by clear stack functions of central type divisible by $\lambda$.

We will prove

(i) the operator $\widetilde{I}_{k}^{\text {o,ss }}$ preserves the filtration (14) by divisibility of partitions,

(ii) on the quotient $\widetilde{K}^{\geq \lambda}(\mathfrak{M})_{(q-1)} / \widetilde{K}^{>\lambda}(\mathfrak{M})_{(q-1)}$, the operator $\widetilde{I}_{k}^{o, s s}$ acts as multiplication by $\widetilde{\mathscr{Q}}_{\lambda}$.

(iii) the operator $\widetilde{I}_{k}^{\text {o,ss }}$ is locally finite.

These facts will imply the claims concerning diagonalizability of $\widetilde{I}_{k}^{\circ, s s}$. This is because for a lower triangular matrix with distinct diagonal entries over a discrete valuation ring to be diagonalizable, it suffices that the differences between the diagonal entries are units. The latter condition is satisfied, because if $\lambda \mid \mu$, then $\widetilde{Q}_{\mu}-\widetilde{Q}_{\lambda}$ does not vanish at $q=1$. (This argument does not apply directly, because our eigenvalues are not linearly ordered, but only partially. Nevertheless, the conclusion remains true in this larger generality.)

Let us fix a partition $\lambda$ of length $k$, and consider a clear stack function $X \rightarrow \mathfrak{M}$ of central type $\lambda$, with algebra $A \rightarrow X$. Abbreviate the induced element of $K^{\geq k} / K^{>k}(\mathfrak{M})$ by $[X]$. Denote the central rank of $X$ by $n$, so that $\lambda \vdash n$. As $A$ has $k$ central idempotents, $E_{k} X \rightarrow X$ has $k$ ! canonical 
sections, each given by a labelling $\sigma$ of these $k$ idempotents. Denote the images of these sections by $\left\{X_{\sigma}\right\}$. By the proof of Theorem 2.4, the algebroid $E_{k} X$ can be stratified as

$$
E_{k} X=\bigsqcup_{\sigma} X_{\sigma} \sqcup \bigsqcup_{\tau} Y_{\tau}
$$

where the $Y_{\tau}$ are clear algebroids of split central rank larger than $k$. The part of $\widetilde{I}_{k}^{\circ, s s} X$ lying over $Y_{\tau}$ then also has split central rank larger than $k$. Hence, when calculating $\widetilde{I}_{k}^{\mathrm{o}, s s}[X]$, we can discard all $Y_{\tau}$. Every $X_{\sigma}$ is isomorphic to $X$, and so we will fix a labelling $\sigma$, and replace $X_{\sigma}$ by $X$ in the following arguments, remembering to multiply the final result by $k$ !.

We need to consider $A^{\times, s s}$ and its quotient $\widetilde{A}^{\times, s s}$. We write

$$
A^{\times, s s}=Z^{\times, s s} \sqcup N Z^{\times, s s},
$$

where $Z \subset A$ is the center of $A$ (which is a strict subbundle and hence a closed substack) and $N Z$ is its complement. We start by examining $Z^{\times, s s}$, and its quotient $\widetilde{Z}^{\times, s s}=Z^{\times, s s} / \mathbb{G}_{m}^{k}$. Note that $Z^{\times, s s}$ and $\widetilde{Z}^{\times, s s}$ are pullbacks from the coarse Deligne-Mumford stack $\bar{X}$ of $X$, and hence are inert over $X$, and their algebroid structures are hence the canonical algebroid structures as inert $X$-stacks.

As in the proof of Theorem 2.4 let $\widetilde{X} \rightarrow X$ be a connected Galois cover with Galois group $\Gamma$, acting on the set $\underline{n}$, such that

$$
\tilde{X} \times_{\Gamma} \underline{n} \stackrel{\simeq}{\longrightarrow} P Z E(A) .
$$

We get induced isomorphisms

$$
\tilde{X} \times_{\Gamma} \mathbb{A}^{n} \stackrel{\simeq}{\longrightarrow} \pi_{*} \mathscr{O}_{P Z E(A)},
$$

and

$$
\tilde{X} \times_{\Gamma} \mathbb{G}_{m}^{n} \stackrel{\simeq}{\longrightarrow}\left(\pi_{*} \mathscr{O}_{P Z E(A)}\right)^{\times} .
$$

By Proposition 1.35 we have a surjective closed immersion

$$
\left(\pi_{*} \mathscr{O}_{P Z E(A)}\right)^{\times, s t r a t} \longrightarrow Z^{\times, s s} .
$$

It follows that we have a surjective closed immersion

$$
\left(\widetilde{X} \times_{\Gamma} \mathbb{G}_{m}^{n}\right)^{\text {strat }} \longrightarrow Z^{\times, s s}
$$

and by passing to the quotient another surjective closed immersion

$$
\left(\tilde{X} \times_{\Gamma} \mathbb{G}_{m}^{n} / \mathbb{G}_{m}^{k}\right)^{\text {strat }} \longrightarrow \widetilde{Z}^{\times, s s} .
$$

So in $K^{\geq k} / K^{>k}(\mathfrak{M})$, we can replace $\widetilde{Z}^{\times, s s}$ by $\widetilde{X} \times_{\Gamma} \mathbb{G}_{m}^{n} / \mathbb{G}_{m}^{k}$.

Our labelling $\sigma$ of the central idempotents in $A_{X}$ corresponds to a labelling of the orbits of $\Gamma$ on $\underline{n}$, and a labelling of the parts of $\lambda \vdash n$. Let us denote these orbits by $I_{1}, \ldots, I_{k}$, and $\lambda$ by $\left(\lambda_{1}, \ldots, \lambda_{k}\right)$, such that $\lambda_{i}=\left|I_{i}\right|$, for $i=1, \ldots, k$. We write $\mathbb{P}\left(\mathbb{A}^{\lambda}\right)$ for the product of projective spaces $\mathbb{P}\left(\mathbb{A}^{\lambda_{1}}\right) \times \ldots \times \mathbb{P}\left(\mathbb{A}^{\lambda_{k}}\right)$. Moreover, for a sequence of subsets $J_{i} \subset I_{i}$ we write $\mathbb{P}^{*}\left(J_{1}, \ldots, J_{k}\right) \subset \mathbb{P}\left(\mathbb{A}^{\lambda}\right)$ for the locally closed subspace defined 
by the entries in $J_{1} \cup \ldots \cup J_{k}$ being non-zero, and all others to be zero. We have

$$
\mathbb{P}\left(\mathbb{A}^{\lambda}\right)=\bigsqcup_{\substack{\left(J_{1}, \ldots, J_{k}\right) \in \\ \mathfrak{P}\left(I_{1}\right) \times \ldots \times \mathfrak{P}\left(I_{k}\right)}} \mathbb{P}^{*}\left(J_{1}, \ldots, J_{k}\right),
$$

where the disjoint union is over all sequences of subsets $J_{i} \subset I_{i}$.

The group $\Gamma$ acts linearly on $\mathbb{P}\left(\mathbb{A}^{\lambda}\right)$, respecting this stratification (although not the individual strata), and we have

$$
\tilde{X} \times_{\Gamma} \mathbb{G}_{m}^{n} / \mathbb{G}_{m}^{k}=\tilde{X} \times_{\Gamma} \mathbb{P}^{*}\left(I_{1}, \ldots, I_{k}\right) .
$$

Moreover,

$$
\begin{aligned}
\tilde{X} \times_{\Gamma} \mathbb{P}\left(\mathbb{A}^{\lambda}\right)= & \tilde{X} \times_{\Gamma} \bigsqcup_{\mathfrak{P}\left(I_{1}\right) \times \ldots \times \mathfrak{P}\left(I_{k}\right)} \mathbb{P}^{*}\left(J_{1}, \ldots, J_{k}\right) \\
= & \bigsqcup_{\mathfrak{P}\left(I_{1}\right) \times \ldots \times \mathfrak{P}\left(I_{k}\right) / \Gamma} \tilde{X} \times_{\operatorname{Stab}\left(J_{1}, \ldots, J_{n}\right)} \mathbb{P}^{*}\left(J_{1}, \ldots, J_{k}\right) \\
= & \tilde{X} \times \Gamma \mathbb{P}^{*}\left(I_{1}, \ldots, I_{k}\right) \sqcup \\
& \bigsqcup_{\substack{\mathfrak{P}\left(I_{1}\right) \times \ldots \times \mathfrak{P}\left(I_{k}\right) / \Gamma \\
\operatorname{Stab}\left(J_{1}, \ldots, J_{n}\right) \subsetneq \Gamma}} \tilde{X} \times_{\operatorname{Stab}\left(J_{1}, \ldots, J_{n}\right)} \mathbb{P}^{*}\left(J_{1}, \ldots, J_{k}\right) .
\end{aligned}
$$

Every subgroup $\Gamma^{\prime} \subsetneq \Gamma$, which is the stabilizer of a sequence $\left(J_{1}, \ldots, J_{k}\right)$, has more than $k$ orbits on $\underline{n}$. As in the proof of Theorem 2.4 this implies that $\tilde{X} / \Gamma^{\prime}$ is in $K^{>k}(\mathfrak{M})$. The same is then true for $\widetilde{X} \times_{\Gamma^{\prime}} \mathbb{P}^{*}\left(J_{1}, \ldots, J_{k}\right)$, as the projection $\tilde{X} \times_{\Gamma^{\prime}} \mathbb{P}^{*}\left(J_{1}, \ldots, J_{k}\right) \rightarrow \widetilde{X} / \Gamma^{\prime}$ is inert (being the pullback of a corresponding morphism of course Deligne-Mumford stacks). We deduce that in $K^{\geq k} / K^{>k}(\mathfrak{M})$, we have

$$
\begin{aligned}
{\left[\widetilde{Z}^{\times, s s}\right] } & =\left[\tilde{X} \times_{\Gamma} \mathbb{G}_{m}^{n} / \mathbb{G}_{m}^{k}\right]=\left[\tilde{X} \times_{\Gamma} \mathbb{P}^{*}\left(I_{1}, \ldots, I_{k}\right)\right]=\left[\tilde{X} \times_{\Gamma} \mathbb{P}\left(\mathbb{A}^{\lambda}\right)\right] \\
& =\left[\mathbb{P}\left(\mathbb{A}^{\lambda}\right)\right][X]=\frac{1}{k !} \widetilde{\mathscr{Q}}_{\lambda}(q)[X]
\end{aligned}
$$

In the last step, we used the bundle relations in $\widetilde{K}(\mathfrak{M})$. The bundle $\widetilde{X} \times_{\Gamma} \mathbb{P}\left(\mathbb{A}^{\lambda}\right)$ is a product of projective bundles associated to vector bundles, whose structure groups are special (as they are general linear groups).

Now consider a locally closed embedding $Y \hookrightarrow N Z^{\times, s s} / \mathbb{G}_{m}^{k}$, such that $Y$ is a clear algebroid. Over $Y$, we then consider the inclusion of commutative algebra bundles $\left.Z A_{X}\right|_{Y} \hookrightarrow Z A_{Y}$. By Proposition 1.36, the split rank of $Z A_{Y}$ (which is the split central rank of $Y$ ), is at least as large as the split rank of $\left.Z A_{X}\right|_{Y}$ which, in turn, is at least as large as the split rank of $Z A_{X}$, which is $k$. Since we are working modulo $K^{>k}(\mathfrak{M})$, we may assume that the split central rank of $Y$ is $k$, and hence that the split rank of $Z A_{Y}$ and of $\left.Z A_{X}\right|_{Y}$ are both equal to $k$. Consider the correspondence $Q$ which we used in the proof of Proposition [1.36]

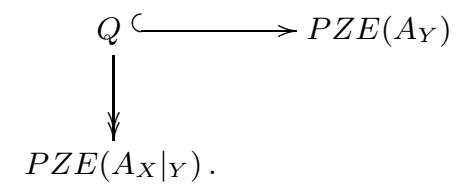


All three stacks in this diagram are representable finite étale covers of $Y$. By assumption, both $P Z E\left(A_{Y}\right)$ and $P Z E\left(\left.A_{X}\right|_{Y}\right)$ have $k$ connected components. This implies that the horizontal inclusion in the diagram is an isomorphism, and that we have a surjective representable finite étale cover

$$
\operatorname{PZE}\left(A_{Y}\right) \longrightarrow P Z E\left(\left.A_{X}\right|_{Y}\right)
$$

Since $P Z E\left(\left.A_{X}\right|_{Y}\right)$ and $P Z E\left(A_{X}\right)$ have the same number of components, the degrees of these components are equal as well, which means that the central type of $\left.A_{X}\right|_{Y}$ is equal to the central type of $A_{X}$, which is $\lambda$. The existence of (17) then implies that $\lambda$ divides the central type of $A_{Y}$.

The surjection (17) is not an isomorphism, because otherwise, by Proposition 1.36 we would have a surjection $\left.Z A_{Y}^{\times, s s} \rightarrow Z A_{X}^{\times, s s}\right|_{Y}$, but this would force the tautological section class of $Z A_{Y}^{\times, s s}$, given by the structure map $Y \rightarrow A_{X}^{\times, s s} / \mathbb{G}_{m}^{k}$ to be central in $A_{X}$ (at least pointwise), which it is not.

This shows that the central type of $Y$ strictly divides $\lambda$.

We have thus completed the proof of (i), and (ii), above. For the local finiteness of $\widetilde{I}_{k}^{\circ, s s}$, proceed as in the proof of Corollary 2.5. Every time we apply $\widetilde{I}_{k}^{\circ, s s}$, we produce only clear algebroids whose central type is a multiple of $\lambda$, but as we can bound the central rank by the rank, which does not increase by applying $\widetilde{I}_{k}^{\circ, s s}$, after finitely many steps, this process stops.

Corollary 2.16. The operator $I^{\circ, s s}: K(\mathfrak{M})_{(q-1)} \rightarrow K(\mathfrak{M})_{(q-1)}$ is diagonalizable. Its eigenvalue spectrum consists of the $\mathscr{Q}_{\lambda} \in \mathbb{Q}[q]$, for all partitions $\lambda$. Denote the eigenspace corresponding to the eigenvalue $\mathscr{Q}_{\lambda}$ by $K^{\lambda}(\mathfrak{M})_{(q-1)}$. We have

$$
K^{k}(\mathfrak{M})_{(q-1)}=\bigoplus_{|\lambda|=k} K^{\lambda}(\mathfrak{M})_{(q-1)}
$$

Example 2.17. Consider, as in Example 2.12 the stack function of rank 2 vector bundles. It defines an element $\left[B G L_{2}\right]$ of $K^{\geq 1} / K^{>1}(\mathfrak{V e c t})$, which we are going to decompose into its eigencomponents with respect to the operator $\widetilde{I}_{1}^{\circ}, s s$.

The stack function $\left[B G L_{2}\right]$ is clear, its central rank is 1 . The decomposition (15) is given in this case as

$$
I_{B G L_{2}}^{\circ, s s}=\Delta / G L_{2} \sqcup T^{*} / N,
$$

where $\Delta$ is the central torus of $G L_{2}$, and $T^{*}=T \backslash \Delta$. Also, $N$ is the normalizer of $T$ in $G L_{2}$. We get the corresponding decomposition

$$
\begin{aligned}
\widetilde{I}_{1}^{\circ, s s} B G L_{2} & =\left(\Delta / \mathbb{G}_{m}\right) / G L_{2} \sqcup\left(T^{*} / \mathbb{G}_{m}\right) / N \\
& =B G L_{2} \sqcup \widetilde{T}^{*} / N,
\end{aligned}
$$

where $\widetilde{T}^{*}=T^{*} / \mathbb{G}_{m}$, and we have

$$
\widetilde{I}_{1}^{\mathrm{o}, s s}\left[B G L_{2}\right]=\left[B G L_{2}\right]+\left[\widetilde{T}^{*} / N\right] .
$$


Note that $\widetilde{T}^{*} / N$ is not a strict algebroid. In fact, let $T^{\prime} \subset T^{*}$ be the closed subscheme consisting of elements of trace zero, and write $T^{*}=$ $T^{\prime} \sqcup T^{* *}$. Then (at least if $2 \in R^{*}$ ) we have $T^{\prime} / \mathbb{G}_{m}=\operatorname{Spec} R$, and $\left[\widetilde{T}^{*} / N\right]=[B N]+\left[\widetilde{T}^{* *} / N\right]$, and $B N$ is not a strict algebroid, as $N$ is not connected. But $\widetilde{T}^{*} / N$ is a clear algebroid. Its connected inertia stack is $\widetilde{T}^{*} \times T / N$, and its associated coarse Deligne-Mumford stack is $\widetilde{T}^{*} / \mathbb{Z}_{2}$, which is only generically a scheme. The central rank of $\widetilde{T}^{*} / N$ is 2 , and the split central rank 1 .

Now we consider $\widetilde{I}_{1}^{\mathrm{o}, s s}\left[\widetilde{T}^{*} / N\right]$. We start by noting that all connected inertia of $\widetilde{T}^{*} / N$ is central. Hence, modulo $K^{>1}(\mathfrak{V e c t})$, we have

$$
\widetilde{I}_{1}^{o, s s}\left[\widetilde{T}^{*} / N\right]=(q+1)\left[\widetilde{T}^{*} / N\right]
$$

by (16).

We see that $\mathbb{Q}[q]_{(q-1)}\left[B G L_{2}\right]+\mathbb{Q}[q]_{(q-1)}\left[\widetilde{T}^{*} / N\right]$ is invariant under $\widetilde{I}_{1}^{\circ, s s}$, and the matrix of $\widetilde{I}_{1}^{\circ, s s}$ on this subspace is

$$
\left(\begin{array}{cc}
1 & 0 \\
1 & q+1
\end{array}\right)
$$

This matrix is lower triangular, and the differences between the scalars on the diagonal are all invertible in $\mathbb{Q}[q]_{(q-1)}$. Therefore it is diagonalizable over $\mathbb{Q}[q]_{(q-1)}$. Diagonalizing, we get the following eigenvectors modulo $K^{>1}(\mathfrak{V} \mathfrak{e c t})_{(q-1)}$ :

(i) $\bar{v}_{(1)}=\left[B G L_{2}\right]-\frac{1}{q}\left[\widetilde{T}^{*} / N\right]$,

(ii) $\bar{v}_{(2)}=\frac{1}{q}\left[\widetilde{T}^{*} / N\right]$.

To get the actual eigenvectors, we project into $K^{1}(\mathfrak{V e c t})_{(q-1)}$. We have

(i) $\pi_{1}\left[B G L_{2}\right]=\left[B G L_{2}\right]-\frac{1}{2}[B T]$

(ii) $\pi_{1}\left[\widetilde{T}^{*} / N\right]=\left[\widetilde{T}^{*} / N\right]-\frac{1}{2}(q-2)[B T]$,

and hence

(i) $v_{(1)}=\left[B G L_{2}\right]-\frac{1}{q}\left[\widetilde{T}^{*} / N\right]-\frac{1}{q}[B T]$,

(ii) $v_{(2)}=\frac{1}{q}\left[\widetilde{T}^{*} / N\right]-\frac{q-2}{2 q}[B T]$.

If we add

(iii) $v_{(1,1)}=\frac{1}{2}\left[B G L_{2}\right]$,

we get the spectral decomposition $\left[B G L_{2}\right]=v_{(1)}+v_{(2)}+v_{(1,1)}$ of $\left[B G L_{2}\right]$, with respect to the operator $I^{\circ, s s}$. This is, of course, the same as the spectral decomposition with respect to $I^{s s}$, which we computed in the introduction (after applying the bundle relations).

Remark 2.18. If we are willing to invert $(q-1)$, we can prove the diagonalizability of $I^{\circ, s s}=I^{s s}$ entirely within the context of strict algebroids. In fact, we can generalize the calculation in the introduction to accomplish this. 


\subsection{Graded structure of multiplication}

We will now assume that $\mathfrak{M}$ admits all direct sums. Then we can define a commutative product on $K(\mathfrak{M})$ by

$$
[X \rightarrow \mathfrak{M}] \cdot[Y \rightarrow \mathfrak{M}]=[X \times Y \rightarrow \mathfrak{M} \times \mathfrak{M} \stackrel{\oplus}{\longrightarrow} \mathfrak{M}]
$$

With this product $K(\mathfrak{M})$ becomes a commutative $K(\mathrm{DM})$-algebra with unit $1=[\operatorname{Spec} R \stackrel{0}{\longrightarrow} \mathfrak{M}]$.

Proposition 2.19. For $x, y \in K(\mathfrak{M})$, we have

$$
I^{\circ, s s}(x \cdot y)=I^{\circ, s s}(x) \cdot I^{\circ, s s}(y) .
$$

Proof. This follows immediately from the fact that, for any two algebroids $X, Y$, we have $A_{X \times Y}^{\times, s s}=A_{X}^{\times, s s} \times A_{Y}^{\times, s s}$, as algebroids over $X \times Y$.

Denote the disjoint union of two partitions $\lambda$ and $\mu$ by $\lambda+\mu$.

Corollary 2.20. We have $K^{\lambda}(\mathfrak{M})_{(q-1)} \cdot K^{\mu}(\mathfrak{M})_{(q-1)} \subset K^{\lambda+\mu}(\mathfrak{M})_{(q-1)}$, and hence also $K^{k}(\mathfrak{M})_{(q-1)} \cdot K^{\ell}(\mathfrak{M})_{(q-1)} \subset K^{k+\ell}(\mathfrak{M})_{(q-1)}$.

So the $\mathbb{Q}[q]_{(q-1)}$-module

$$
K(\mathfrak{M})_{(q-1)}=\bigoplus_{k \geq 0} K^{k}(\mathfrak{M})_{(q-1)}
$$

is a graded $\mathbb{Q}[q]_{(q-1)}$-algebra, with respect to the commutative product on $K(\mathfrak{M})_{(q-1)}$. We will prove next, that this fact is true for $K(\mathfrak{M})$ itself.

Proposition 2.21. For any $x, y \in K(\mathfrak{M})$ and any $p \geq 0$, we have

$$
E_{p}(x \cdot y)=\sum_{n, m}\left[\begin{array}{c}
p \\
n, m
\end{array}\right] E_{n}(x) \cdot E_{m}(y) .
$$

Here $\left[\begin{array}{c}p \\ n, m\end{array}\right]$ is the number of ways the set $\underline{p}$ can be written as the union of a subset of order $n$, and a subset of order $m$.

Proof. Consider stack functions $X \rightarrow \mathfrak{M}$ and $Y \rightarrow \mathfrak{M}$. Then $E_{p}(X \times Y)$ is the stack of pairs $(e, f)$, where $e=\left(e_{\rho}\right)_{\rho \in \underline{p}}$ is a complete set of orthogonal idempotents in $A_{X}$, and $f=\left(f_{\rho}\right)_{\rho \in \underline{p}}$ is a complete set of orthogonal idempotents in $A_{Y}$, such that for every $\rho=1, \ldots, p$, at least one of the two idempotents $e_{\rho}, f_{\rho}$ is non-zero.

For every pair of strictly monotone maps $\underline{n} \hookrightarrow \underline{p}, \underline{m} \hookrightarrow \underline{p}$, whose images cover $\underline{p}$, we get a morphism of stack functions $\bar{E}_{n}(\bar{X}) \times \bar{E}_{m}(Y) \rightarrow$ $E_{p}(X \times Y)$, by mapping a pair of complete sets of orthogonal idempotents $\left(e^{\prime}, f^{\prime}\right)$, where $e^{\prime}=\left(e_{\nu}^{\prime}\right)_{\nu \in \underline{n}}$ and $f^{\prime}=\left(f_{\mu}^{\prime}\right)_{\mu \in \underline{m}}$, to the pair $(e, f)$, defined by

$$
e_{\rho}=\sum_{\nu \mapsto \rho} e_{\nu}^{\prime} \quad \text { and } \quad f_{\rho}=\sum_{\mu \mapsto \rho} f_{\mu}^{\prime} .
$$

(As the maps $\underline{n} \rightarrow \underline{p}$ and $\underline{m} \rightarrow \underline{p}$ are injective, all these sums have either zero or one summand.) 
Each of the morphisms $E_{n}(X) \times E_{m}(X) \rightarrow E_{p}(X \times Y)$ is an isomorphism onto a locally closed substack, because the locus of vanishing for an idempotent is closed. Moreover, the images of these morphisms are disjoint, and from a cover. There are $\left[\begin{array}{c}p \\ n, m\end{array}\right]$ of them.

Corollary 2.22. If $x \in K^{k}(\mathfrak{M})$ and $y \in K^{\ell}(\mathfrak{M})$, then $x \cdot y \in K^{k+\ell}(\mathfrak{M})$.

Proof. We have

$$
\begin{aligned}
\pi_{t}(x \cdot y) & =\sum_{p}\left(\begin{array}{l}
t \\
p
\end{array}\right) E_{p}(x \cdot y) \\
& =\sum_{p}\left(\begin{array}{l}
t \\
p
\end{array}\right) \sum_{n, m}\left[\begin{array}{c}
p \\
n, m
\end{array}\right] E_{n}(x) \cdot E_{m}(y) \\
& =\sum_{n, m}\left(\sum_{p}\left[\begin{array}{c}
p \\
n, m
\end{array}\right]\left(\begin{array}{l}
t \\
p
\end{array}\right)\right) E_{n}(x) \cdot E_{m}(y) \\
& =\sum_{n, m}\left(\begin{array}{c}
t \\
n
\end{array}\right)\left(\begin{array}{c}
t \\
m
\end{array}\right) E_{n}(x) \cdot E_{m}(x) \\
& =\pi_{t}(x) \cdot \pi_{t}(y)
\end{aligned}
$$

The step from Line 3 to Line 4 uses Proposition 2.23 below.

\section{A combinatorial lemma}

Let $p \geq 0$.

For a non-negative integer $n$, and a $p$-tuple of non-negative integers $\lambda=\left(\lambda_{1}, \ldots, \lambda_{p}\right)$, we define

$$
\left[\begin{array}{l}
n \\
\lambda
\end{array}\right]=\left[\begin{array}{c}
n \\
\lambda_{1}, \ldots, \lambda_{p}
\end{array}\right]
$$

to be the number of indexed covers of $\underline{n}$ by subsets $S_{1}, \ldots, S_{p}$ of cardinalities $\lambda_{1}, \ldots, \lambda_{p}$. The non-negative integer $\left[\begin{array}{l}n \\ \lambda\end{array}\right]$ vanishes, unless $\lambda_{\rho} \leq n$, for all $\rho=1, \ldots, p$, and $n \leq|\lambda|$, where $|\lambda|=\sum_{\rho} \lambda_{\rho}$.

We have the following useful combinatorial property:

Proposition 2.23. For every $p$-tuple of non-negative integers $\lambda=\left(\lambda_{1}, \ldots, \lambda_{p}\right)$, we have

$$
\left(\begin{array}{c}
t \\
\lambda_{1}
\end{array}\right) \cdots\left(\begin{array}{c}
t \\
\lambda_{p}
\end{array}\right)=\sum_{n}\left[\begin{array}{l}
n \\
\lambda
\end{array}\right]\left(\begin{array}{l}
t \\
n
\end{array}\right)
$$

Proof. Let $x_{1}, \ldots, x_{p}$ be formal variables. We will prove that

$$
\sum_{\lambda}\left(\begin{array}{c}
t \\
\lambda_{1}
\end{array}\right) \ldots\left(\begin{array}{c}
t \\
\lambda_{p}
\end{array}\right) x_{1}^{\lambda_{1}} \ldots x_{p}^{\lambda_{p}}=\sum_{\lambda} \sum_{n}\left[\begin{array}{c}
n \\
\lambda
\end{array}\right]\left(\begin{array}{c}
t \\
n
\end{array}\right) x_{1}^{\lambda_{1}} \ldots x_{p}^{\lambda_{p}}
$$


by proving that both sides of this equation are equal to

$$
\prod_{i=1}^{p}\left(1+x_{i}\right)^{t}
$$

On the one hand, we have

$$
\begin{aligned}
\prod_{i=1}^{p}\left(1+x_{i}\right)^{t} & =\prod_{i=1}^{p} \sum_{n}\left(\begin{array}{c}
t \\
n
\end{array}\right) x_{i}^{n} \\
& =\sum_{\lambda_{1}, \ldots, \lambda_{p}}\left(\begin{array}{c}
t \\
\lambda_{1}
\end{array}\right) \cdots\left(\begin{array}{c}
t \\
\lambda_{p}
\end{array}\right) x_{1}^{\lambda_{1}} \ldots x_{p}^{\lambda_{p}} .
\end{aligned}
$$

On the other hand, we have

$$
\begin{aligned}
\prod_{i=1}^{p}\left(1+x_{i}\right)^{t} & =\left(1+\prod_{i=1}^{p}\left(1+x_{i}\right)-1\right)^{t} \\
& =\sum_{n}\left(\begin{array}{c}
t \\
n
\end{array}\right)\left(\prod_{i=1}^{p}\left(1+x_{i}\right)-1\right)^{n} \\
& =\sum_{n}\left(\begin{array}{c}
t \\
n
\end{array}\right) \sum_{j}(-1)^{j}\left(\begin{array}{l}
n \\
j
\end{array}\right) \prod_{i=1}^{p}\left(1+x_{i}\right)^{n-j} \\
& =\sum_{n}\left(\begin{array}{c}
t \\
n
\end{array}\right) \sum_{j}(-1)^{j}\left(\begin{array}{l}
n \\
j
\end{array}\right) \prod_{i=1}^{p} \sum_{\ell}\left(\begin{array}{c}
n-j \\
\ell
\end{array}\right) x_{i}^{\ell} \\
& =\sum_{n}\left(\begin{array}{c}
t \\
n
\end{array}\right) \sum_{j}(-1)^{j}\left(\begin{array}{c}
n \\
j
\end{array}\right) \sum_{\lambda_{1}, \ldots, \lambda_{p}}\left(\begin{array}{c}
n-j \\
\lambda_{i}
\end{array}\right) x_{1}^{\lambda_{1}} \ldots x_{p}^{\lambda_{p}} \\
& =\sum_{n}\left(\begin{array}{c}
t \\
n
\end{array}\right) \sum_{\lambda}\left(\sum_{j}(-1)^{j}\left(\begin{array}{c}
n \\
j
\end{array}\right)\left(\begin{array}{c}
n-j \\
\lambda_{i}
\end{array}\right)\right) x^{\lambda} \\
& =\sum_{n}\left(\begin{array}{c}
t \\
n
\end{array}\right) \sum_{\lambda}\left[\begin{array}{c}
n \\
\lambda_{1}, \ldots, \lambda_{p}
\end{array}\right] x^{\lambda} \\
& =\sum_{\lambda} \sum_{n}\left[\begin{array}{l}
n \\
\lambda
\end{array}\right]\left(\begin{array}{c}
t \\
n
\end{array}\right) x^{\lambda} .
\end{aligned}
$$

Here we have used the obvious inclusion-exclusion property satisfied by the covering numbers. $\square$ 


\section{The order filtration}

\section{The Hall algebra}

Let $\mathfrak{M}$ be a linear algebraic stack admitting direct sums and direct summands, i.e., assume that $\mathfrak{M}$ is Karoubian (Remark 1.63). To define the Hall product, we need an additional structure on $\mathfrak{M}$. This is a linear algebraic substack $\mathfrak{M}^{(2)}$ of the stack of all sequences $M^{\prime} \rightarrow M \rightarrow M^{\prime \prime}$ in $\mathfrak{M}$, such that for every $R$-scheme $S$, the fibre $\mathfrak{M}^{(2)}(S)$ defines the structure of an exact category on $\mathfrak{M}(S)$. The stack $\mathfrak{M}^{(2)}$ comes with a diagram of morphisms of linear algebraic stacks

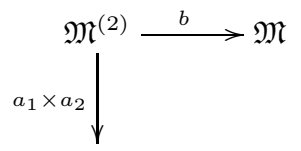

$\mathfrak{M} \times \mathfrak{M}$

where $a_{1}, a_{2}, b: \mathfrak{M}^{(2)} \rightarrow \mathfrak{M}$ are the projections of the sequence $M^{\prime} \rightarrow$ $M \rightarrow M^{\prime \prime}$ onto the objects $M^{\prime}, M^{\prime \prime}, M$, respectively. We require further, that the morphism $a_{1} \times a_{2}: \mathfrak{M}^{(2)} \rightarrow \mathfrak{M} \times \mathfrak{M}$ is of finite type, and the morphism $b: \mathfrak{M}^{(2)} \rightarrow \mathfrak{M}$ is representable.

We call such an $\mathfrak{M}$ an exact linear algebraic stack.

Example 3.1. The linear stacks $\mathfrak{C o h}_{X}, \mathfrak{V e c t}$, and $\mathfrak{R e p} \mathfrak{p}_{Q}$ of Examples 1.11 1.12 and 1.13 satisfy these axioms. For $\mathfrak{C o h}_{X}$, see [5. Section 4.1].

In each case, the exact structure is given by all short exact sequences. Note that the categories $\mathfrak{M}(S)$ are not abelian, as the cokernel of a homomorphisms of flat sheaves is not necessarily flat.

Throughout the following discussion we fix an exact linear algebraic stack $\mathfrak{M}$, and let $\mathfrak{A} \rightarrow \mathfrak{M}$ be its universal endomorphism algebra, as in Section 2

We have the following structures on $K(\mathfrak{M})$.

1. Module structure. The action of $K(\mathrm{DM})$ on $K(\mathfrak{M})$, given by $[Z] \cdot[X \rightarrow \mathfrak{M}]=[Z \times X \rightarrow X \rightarrow \mathfrak{M}]$, which turns $K(\mathfrak{M})$ into a $K(\mathrm{DM})$-module.

2. Multiplication. The commutative multiplication given by

$$
[X \rightarrow \mathfrak{M}] \cdot[Y \rightarrow \mathfrak{M}]=[X \times Y \rightarrow \mathfrak{M} \times \mathfrak{M} \stackrel{\oplus}{\longrightarrow} \mathfrak{M}]
$$

3. Hall product. The Hall product of the stack functions $[X \rightarrow \mathfrak{M}]$ and $[Y \rightarrow \mathfrak{M}]$, which is defined by first constructing the fibered product

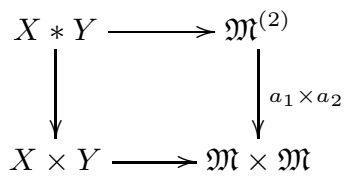

and then setting

$$
[X \rightarrow \mathfrak{M}] *[Y \rightarrow \mathfrak{M}]=\left[X * Y \longrightarrow \mathfrak{M}^{(2)} \stackrel{b}{\longrightarrow} \mathfrak{M}\right]
$$


The multiplication is associative and commutative, the Hall product is associative. The unit with respect to both multiplications is given by the 0 -object of $\mathfrak{M}$ :

$$
1=[\operatorname{Spec} R \stackrel{0}{\longrightarrow} \mathfrak{M}] .
$$

From now on we will refer to $K(\mathfrak{M})$ as the Hall algebra of $\mathfrak{M}$.

\subsection{Filtered structure of the Hall algebra}

Definition 3.2. For $n \geq 0$, we define

$$
K^{\leq n}(\mathfrak{M})=\operatorname{ker} E_{n+1}=\bigoplus_{k \leq n} K^{k}(\mathfrak{M})
$$

This is an ascending filtration on $K(\mathfrak{M})$, called the filtration by the order of vanishing of inertia at $q=1$, or simply the order filtration of $K(\mathfrak{M})$.

This is a slight abuse of language, because only the space obtained by extension of scalars $K^{\leq n}(\mathfrak{M})_{(q-1)}$ is the direct sum of all eigenspaces of $I^{\circ, s s}$ whose corresponding eigenvalues $\mathscr{Q} \in \mathbb{Q}[q]$ have order of vanishing at $q=1$ less than or equal to $n$.

Theorem 3.3. Suppose that $\xi \in K^{\leq n}(\mathfrak{M})$ and $\chi \in K^{\leq m}(\mathfrak{M})$, then $\xi * \eta \in$ $K^{\leq n+m}(\mathfrak{M})$. Moreover, we have

$$
\xi * \chi \equiv \xi \cdot \chi \quad \bmod K^{<n+m} .
$$

To prove this theorem we will prove the following lemma.

Lemma 3.4. For any two stack functions $\xi, \chi \in K(\mathfrak{M})$, and for any integer $p \geq 0$, we have

$$
\frac{1}{p !} E_{p}\left(\pi_{t}(\xi) * \pi_{t}(\chi)\right) \equiv \sum_{i+j=p} \pi_{i}(\xi) \pi_{j}(\chi) t^{p} \bmod t^{p+1}
$$

as an equation in $K(\mathfrak{M})[t]$.

Before proving the lemma, let us indicate how the lemma implies the theorem. For this, suppose that $\xi \in K^{\leq k}(\mathfrak{M})$ and $\chi \in K^{\leq \ell}(\mathfrak{M})$. Then the degree of $\pi_{t}(\xi)$ in $t$ is at most $k$ and the degree of $\pi_{t}(\chi)$ is at most $\ell$. So the degree of $E_{p}\left(\pi_{t}(\xi) * \pi_{t}(\chi)\right)$ is at most $k+\ell$. So we see that if $p>k+\ell$, then $E_{p}\left(\pi_{t}(\xi) * \pi_{t}(\chi)\right)=0$, which implies that $\xi * \chi \in K^{\leq k+\ell}(\mathfrak{M})$, by Corollary 2.7

Now set $p=k+\ell$. The left hand side of (19) has degree at most $k+\ell$, the the right hand side has degree exactly $k+\ell$, which implies that both sides are homogeneous of degree $k+\ell$, and we have

$$
\frac{1}{(k+\ell) !} E_{k+\ell}\left(\pi_{t}(\xi) * \pi_{t}(\chi)\right)=\pi_{k}(\xi) \pi_{\ell}(\chi) t^{k+\ell} .
$$

Now notice that if $x \in K^{\leq n}(\mathfrak{M})$, we have $\pi_{n}(x)=\frac{1}{n !} E_{n}(x)$. Hence we can rewrite our equation as

$$
\pi_{k+\ell}\left(\pi_{t}(\xi) * \pi_{t}(\chi)\right)=\pi_{k}(\xi) t^{k} \pi_{\ell}(\chi) t^{\ell} .
$$

This proves the theorem. 
Analysis of $E_{p}\left(E_{n} * E_{m}\right)$

Suppose $\xi=(X \rightarrow \mathfrak{M})$ and $\chi=(Y \rightarrow \mathfrak{M})$ are stack functions. The stack function $\xi * \chi$ is defined by the cartesian diagram:

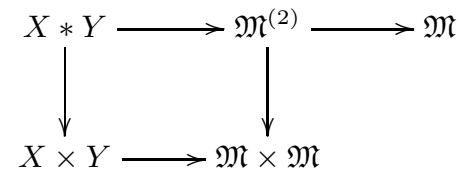

Explicitly, $X * Y$ is the stack of triples $(x, M, y)$,

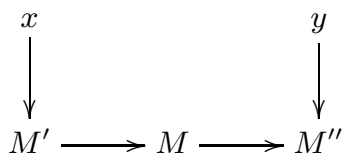

where $x$ and $y$ are objects of $X$ and $Y$, respectively, $M$ is an object of $\mathfrak{M}^{(2)}$, i.e., a short exact sequence $M^{\prime} \rightarrow M \rightarrow M^{\prime \prime}$ of objects in $\mathfrak{M}$, and $x \rightarrow M^{\prime}$ and $y \rightarrow M^{\prime \prime}$ are isomorphisms from the images of $x$ and $y$ in $\mathfrak{M}$ to $M^{\prime}$ and $M^{\prime \prime}$, respectively. (We omit these isomorphisms from the triple to simplify the notation.)

The stack function $E_{n}(\xi) * E_{m}(\chi)$ is defined by the enlarged diagram:

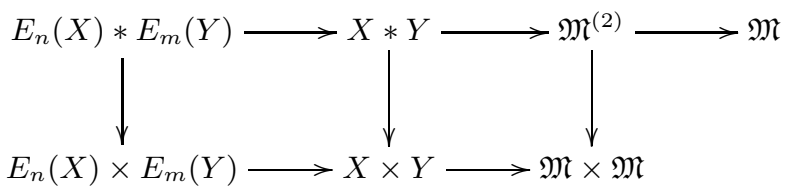

Explicitly, $E_{n}(X) * E_{m}(Y)$ is the stack of 5-tuples $\left(x,\left(e_{\nu}\right), M, y,\left(f_{\mu}\right)\right)$, where $(x, M, y)$ represents a diagram (20), and $\left(e_{\nu}\right)=\left(e_{1}, \ldots, e_{n}\right)$ is a complete set of non-zero orthogonal idempotents in $A(x)$, and $\left(f_{\mu}\right)=$ $\left(f_{1}, \ldots, f_{m}\right)$ is a complete set of non-zero orthogonal idempotents in $A(y)$.

Finally, the stack $E_{p}\left(E_{n}(X) * E_{m}(Y)\right)$ is the stack of objects of $E_{n}(X) *$ $E_{m}(Y)$, endowed with a complete set of $p$ non-zero labelled idempotents. Explicitly, it consists of 6 -tuples

$$
\left(x,\left(e_{\nu, \rho}\right), M,\left(g_{\rho}\right), y,\left(f_{\mu, \rho}\right)\right),
$$

where $(x, M, y)$ is as in (20), and $\left(g_{\rho}\right)_{\rho \in \underline{p}}$ is a complete set of non-zero orthogonal idempotent endomorphisms of the short exact sequence $M^{\prime} \rightarrow$ $M \rightarrow M^{\prime \prime}$. Moreover, $\left(e_{\rho, \nu}\right)_{\rho \in p, \nu \in \underline{n}}$ is a $p n$-tuple of orthogonal idempotents in $A(x)$, and $\left(f_{\rho, \mu}\right)_{\rho \in p, \mu \in \underline{m}}$ is a $p m$-tuple of orthogonal idempotents in $A(y)$, such that for every $\bar{\rho}=1, \ldots, p$ we have $\sum_{\nu=1}^{n} e_{\rho, \nu}=\left.g_{\rho}\right|_{M^{\prime}}$ and $\sum_{\mu=1}^{m} f_{\rho, \mu}=\left.g_{\rho}\right|_{M^{\prime \prime}}$. Finally, we require for all $\nu=1, \ldots, n$ that $e_{\nu}=\sum_{\rho=1}^{p} e_{\rho, \nu} \neq 0$ and for all $\mu=1, \ldots, m$ that $f_{\mu}=\sum_{\rho=1}^{p} f_{\rho, \mu} \neq 0$.

Decomposing $E_{p}\left(E_{n} * E_{m}\right)$

Given $p$-tuples of non-negative integers $\varphi=\left(\varphi_{1}, \ldots, \varphi_{p}\right)$ and $\psi=\left(\psi_{1} \ldots, \psi_{p}\right)$, we define a new stack function $(X * Y)_{\varphi, \psi} \rightarrow \mathfrak{M}$, denoted $(\xi * \chi)_{\varphi, \psi}$, as follows. 
Let $(X * Y)_{\varphi, \psi}$ be the algebraic stack of 6 -tuples

$$
\left(x,\left(e_{\rho}\right), M,\left(g_{\rho}\right), y,\left(f_{\rho}\right)\right),
$$

where $(x, M, y)$ is as in (20), and $\left(g_{\rho}\right)_{\rho=1, \ldots, p}$ is a complete set of nonzero orthogonal idempotent endomorphisms of the short exact $M$. Moreover, for every $\rho=1, \ldots, p$, we require that $e_{\rho}=\left(e_{1}, \ldots, e_{\varphi_{\rho}}\right)$ and $f_{\rho}=\left(f_{1}, \ldots, f_{\psi_{\rho}}\right)$ are families of non-zero orthogonal idempotents for $x$ and $y$, respectively, such that for all $\rho=1, \ldots, p$,

$$
\left.g_{\rho}\right|_{M^{\prime}}=\sum_{\omega=1}^{\varphi_{\rho}} e_{\omega} \quad \text { and }\left.\quad g_{\rho}\right|_{M^{\prime \prime}}=\sum_{\eta=1}^{\psi_{\rho}} f_{\eta} .
$$

It follows that the union of $e_{1}, \ldots, e_{p}$ is a complete set of orthogonal idempotents for $x$, and the union of $f_{1}, \ldots, f_{p}$ is a complete set of orthogonal idempotents for $y$.

There is a natural algebroid structure on $(X * Y)_{\varphi, \psi}$. The morphism to $\mathfrak{M}$ given by mapping the 6 -tuple (22) to the middle object $b(M)$ of the short exact sequence $M$, makes $(X * Y)_{\varphi, \psi}$ into a stack function.

Note that if for some $\rho=1, \ldots, p$ both integers $\varphi_{\rho}$ and $\psi_{\rho}$ vanish, then $(X * Y)_{\varphi, \psi}=\varnothing$, because all $g_{\rho}$ are required to be non-zero.

Let us write $|\varphi|=\sum_{\rho} \varphi_{\rho}$ and $|\psi|=\sum_{\rho} \psi_{\rho}$. Let us assume that for every $\rho=1, \ldots, p$, at least one of the two integers $\varphi_{\rho}, \psi_{\rho}$ is non-zero. Then we have a morphism

$$
E_{|\varphi|}(X) \times E_{|\psi|}(Y) \longrightarrow(X * Y)_{\varphi, \psi}
$$

which maps a quadruple $\left(x,\left(e_{\omega}\right), y,\left(f_{\eta}\right)\right)$ to the 6 -tuple (22) where $M=$ $M^{\prime} \oplus M^{\prime \prime}$, with $M^{\prime}$ denoting the image of $x$ in $\mathfrak{M}$, and $M^{\prime \prime}$ the image of $y$ in $\mathfrak{M}$. To define (24), we break up the complete family of orthogonal idempotents $e_{1}, \ldots, e_{|\varphi|}$ for $x$ into $p$ subfamilies, where the $\rho$-th subfamily has $\varphi_{\rho}$ members. Similarly, we break up $f_{1}, \ldots, f_{|\psi|}$ into $p$ subfamilies whose sizes are $\psi_{1}, \ldots, \psi_{p}$. Then the family of idempotents $\left(g_{\rho}\right)$ on $M$ is defined by formulas (23). Note that we need to make our assumption on the $p$-tuples $\varphi, \psi$, in order for every family member $g_{\rho}$ to be non-zero.

Lemma 3.5. If for every $\rho=1, \ldots, p$ exactly one of the two integers $\varphi_{\rho}$, $\psi_{\rho}$ is non-zero, (24) is an isomorphism. Hence we have the equality

$$
(\xi * \chi)_{\varphi, \psi}=E_{|\varphi|}(\xi) E_{|\psi|}(\chi)
$$

for stack functions.

Proof. Given an object (22) of $(X * Y)_{\varphi, \psi}$, the short exact sequence $M$ is split into a direct sum of $p$ short exact sequences. Each one of these sequences is canonically split, because either the subobject or the quotient object vanishes, by the assumption on $\varphi$ and $\psi$. Therefore the sequence $M$ is split, canonically, too.

Now suppose given strictly monotone maps

$$
\Phi_{\rho}: \underline{\varphi_{\rho}} \longleftrightarrow \underline{n} \quad \text { and } \quad \Psi_{\rho}: \underline{\psi_{\rho}} \longleftrightarrow \underline{m},
$$


for all $\rho=1, \ldots, p$, such that the images of the $\Phi_{\rho}$ cover $\underline{n}$, and the images of the $\Psi_{\rho}$ cover $\underline{m}$. The choice of these injections determines a morphism of algebraic stacks

$$
(X * Y)_{\varphi, \psi} \longrightarrow E_{p}\left(E_{n}(X) * E_{m}(Y)\right),
$$

by mapping the 6-tuple (22) to the 6-tuple (21) by defining

$$
e_{\nu, \rho}=\sum_{\Phi_{\rho}(\omega)=\nu}\left(e_{\rho}\right)_{\omega} \quad \text { and } \quad f_{\mu, \rho}=\sum_{\Psi(\eta)=\mu}\left(f_{\rho}\right)_{\eta} .
$$

By our assumptions, these sums are either empty or consist of a single summand, so the $e_{\nu, \rho}$ and the $f_{\mu, \rho}$ are obtained from the $\left(e_{\rho}\right)_{\omega}$ and the $\left(f_{\rho}\right)_{\eta}$ essentially by relabelling.

Note that the requirements $\bigcup_{\rho} \Phi_{\rho}\left(\underline{\varphi_{\rho}}\right)=\underline{n}$ and $\bigcup_{\rho} \Psi_{\rho}\left(\underline{\psi_{\rho}}\right)=\underline{m}$ are needed to assure that $\sum_{\rho} e_{\nu, \rho}$ and $\sum_{\rho} f_{\mu, \rho}$ are non-zero, for all $\nu=$ $1, \ldots, n$, and $\mu=1, \ldots m$.

Lemma 3.6. The morphism 25) gives rise to a morphism of stack functions $(\xi * \chi)_{\varphi, \psi} \rightarrow E_{p}\left(E_{n}(\xi) * E_{m}(\chi)\right)$, which is both an open and a closed immersion.

If we change any of $\varphi, \psi$, or $\Phi, \Psi$, we get a morphism with disjoint image. The images of all morphisms (25) cover $E_{p}\left(E_{n}(X) * E_{m}(Y)\right)$.

Proof. This follows from the fact that the source and target of (25) only differ in the way the idempotents in $A_{x}$ and $A_{y}$ are indexed.

Corollary 3.7. Using the notation introduced in (18), we have the following equation in $K(\mathfrak{M})$ :

$$
E_{p}\left(E_{n}(\xi) * E_{m}(\chi)\right)=\sum_{\varphi, \psi} \sum_{\Phi, \Psi}(\xi * \eta)_{\varphi, \psi}=\sum_{\varphi, \psi}\left[\begin{array}{l}
n \\
\varphi
\end{array}\right]\left[\begin{array}{c}
m \\
\psi
\end{array}\right](\xi * \eta)_{\varphi, \psi},
$$

where $\varphi, \psi$ run over all $p$-tuples of non-negative integers.

For example, consider $\chi=1$, and $m=0$. If any of the $\psi_{\rho}$ is non-zero, $(X * Y)_{\varphi, \psi}$ is empty. Hence

$$
E_{p} E_{n}(\xi)=\sum_{\varphi_{1}, \ldots, \varphi_{p}>0}\left[\begin{array}{l}
n \\
\varphi
\end{array}\right] E_{|\varphi|}(\xi)
$$

where the sum is over all $p$-tuples of positive integers. 


\section{Proof of the main lemma}

Using Proposition 2.23, we can now calculate as follows:

$$
\begin{aligned}
E_{p}\left(\pi_{t}(\xi) * \pi_{t}(\chi)\right) & =E_{p}\left(\sum_{n}\left(\begin{array}{c}
t \\
n
\end{array}\right) E_{n}(\xi) * \sum_{m}\left(\begin{array}{c}
t \\
m
\end{array}\right) E_{m}(\chi)\right) \\
& =\sum_{n, m}\left(\begin{array}{c}
t \\
n
\end{array}\right)\left(\begin{array}{c}
t \\
m
\end{array}\right) \sum_{\varphi, \psi}\left[\begin{array}{c}
n \\
\varphi
\end{array}\right]\left[\begin{array}{c}
m \\
\psi
\end{array}\right](\xi * \chi)_{\varphi, \psi} \\
& =\sum_{\varphi, \psi}\left(\sum_{n}\left(\begin{array}{c}
t \\
n
\end{array}\right)\left[\begin{array}{c}
n \\
\varphi
\end{array}\right]\right)\left(\sum_{m}\left(\begin{array}{c}
t \\
m
\end{array}\right)\left[\begin{array}{c}
m \\
\psi
\end{array}\right]\right)(\xi * \chi)_{\varphi, \psi} \\
& =\sum_{\varphi, \psi}\left(\begin{array}{c}
t \\
\varphi_{1}
\end{array}\right) \cdots\left(\begin{array}{c}
t \\
\varphi_{p}
\end{array}\right)\left(\begin{array}{c}
t \\
\psi_{1}
\end{array}\right) \cdots\left(\begin{array}{c}
t \\
\psi_{p}
\end{array}\right)(\xi * \chi)_{\varphi, \psi} .
\end{aligned}
$$

For example, if $\chi=1$, we get

$$
E_{p} \pi_{t}(\xi)=\sum_{\varphi_{1}, \ldots, \varphi_{p}>0}\left(\begin{array}{c}
t \\
\varphi_{1}
\end{array}\right) \cdots\left(\begin{array}{c}
t \\
\varphi_{p}
\end{array}\right) E_{|\varphi|}(\xi) .
$$

The lowest order term in (26) has degree $p$, since for $(\xi * \chi)_{\varphi, \psi}$ not to vanish, we need for every $\rho=1, \ldots, p$ at least one of $\varphi_{\rho}, \psi_{\rho}$ to be non-zero.

Modulo $\left(t^{p+1}\right)$, only terms corresponding to pairs $(\varphi, \psi)$, with the property that for every $\rho=1, \ldots, p$ exactly one of $\varphi_{\rho}, \psi_{\rho}$ is non-zero, contribute to (26). These are exactly the terms to which Lemma 3.5 applies, and we deduce, that modulo $t^{p+1}$, we have:

$$
E_{p}\left(\pi_{t}(\xi) * \pi_{t}(\chi)\right) \equiv \sum_{\varphi, \psi}\left(\begin{array}{c}
t \\
\varphi_{1}
\end{array}\right) \ldots\left(\begin{array}{c}
t \\
\varphi_{p}
\end{array}\right)\left(\begin{array}{c}
t \\
\psi_{1}
\end{array}\right) \ldots\left(\begin{array}{c}
t \\
\psi_{p}
\end{array}\right) E_{|\varphi|}(\xi) E_{|\psi|}(\chi),
$$

where the sum is over all $(\varphi, \psi)$, where the supports of $\varphi$ and $\psi$ form a partition of $\underline{p}$. By grouping terms corresponding to partitions of the same size together, we can rewrite this as

$$
\sum_{i+j=p}\left(\begin{array}{c}
p \\
i
\end{array}\right) \sum_{\varphi_{1}, \ldots, \varphi_{i}>0}\left(\begin{array}{c}
t \\
\varphi_{1}
\end{array}\right) \cdots\left(\begin{array}{c}
t \\
\varphi_{i}
\end{array}\right) E_{|\varphi|}(\xi) \sum_{\psi_{1}, \ldots, \psi_{j}>0}\left(\begin{array}{c}
t \\
\psi_{1}
\end{array}\right) \cdots\left(\begin{array}{c}
t \\
\psi_{j}
\end{array}\right) E_{|\psi|}(\chi),
$$

which is equal to

$$
\sum_{i+j=p}\left(\begin{array}{c}
p \\
i
\end{array}\right) E_{i} \pi_{t}(\xi) E_{j} \pi_{t}(\chi)
$$

by (27). Modulo $t^{p+1}$, this term is congruent to

$$
\sum_{i+j=p}\left(\begin{array}{c}
p \\
i
\end{array}\right) E_{i} \pi_{i}(\xi) E_{j} \pi_{j}(\chi)=p ! \sum_{i+j=p} \pi_{i}(\xi) \pi_{j}(\chi) t^{p}
$$

We conclude that

$$
\frac{1}{p !} E_{p}\left(\pi_{t}(\xi) * \pi_{t}(\chi)\right) \equiv \sum_{i+j=p} \pi_{i}(\xi) \pi_{j}(\chi) t^{p} \quad \bmod t^{p+1}
$$

which proves Lemma 3.4 


\subsection{The semi-classical Hall algebra}

By Theorem 3.3, the submodule

$$
\mathscr{K}(\mathfrak{M})=\bigoplus_{n \geq 0} t^{n} K^{\leq n}(\mathfrak{M})
$$

of $K(\mathfrak{M})[t]$ is a $K(\mathrm{DM})[t]$-subalgebra with respect to the Hall product. The algebra $\mathscr{K}(\mathfrak{M})$ is a one-parameter flat family of algebras. The special fibre at $t=0$ is canonically isomorphic to the graded algebra associated to the filtered algebra $(K(\mathfrak{M}), *)$. The quotient map $\mathscr{K} \rightarrow \mathscr{K} / t \mathscr{K}$ is identified with the map $\sum_{n} x_{n} t^{n} \mapsto \sum_{n} \pi_{n}\left(x_{n}\right)$.

The graded algebra associated to the filtered algebra $(K(\mathfrak{M}), *)$, is canonically isomorphic to the commutative graded algebra $(K(\mathfrak{M}), \cdot)$, by Theorem 3.3 The special fibre inherits therefore a Poisson bracket, which encodes the Hall product to second order. This Poisson bracket has degree -1 , and is given by the formula

$$
\{x, y\}=\pi_{k+\ell-1}(x * y-y * x), \quad \text { for } x \in K^{k}(\mathfrak{M}), y \in K^{\ell}(\mathfrak{M}) \text {. }
$$

Corollary 3.8. The graded $K(\mathrm{DM})$-algebra $(K(\mathfrak{M}), \cdot)$ is endowed with a Poisson bracket of degree -1 , given by (28).

Corollary 3.9. In particular, $K^{1}(\mathfrak{M})$ is a Lie algebra with respect to the Poisson bracket (28). In fact, for $x, y \in K^{1}(\mathfrak{M})$, we have that $x * y-y * x \in$ $K^{1}(\mathfrak{M})$, so in this case, the Poisson bracket is equal to the Lie bracket. Thus, $K^{1}(\mathfrak{M})$ is a Lie algebra over the ring of scalars $K(\mathrm{DM})$.

Proof. Equation (26) for $p=0$, together with Lemma 3.5 says

$$
E_{0}\left(\pi_{t}(x) * \pi_{t}(y)\right)=E_{0}(x) E_{0}(y) .
$$

This proves that $E_{0}(x)=0$ or $E_{0}(y)=0$ implies that $E_{0}(x * y)=0$.

Definition 3.10. We call $K^{1}(\mathfrak{M})$ the Lie algebra of virtually indecomposable stack functions. We will usually write $K^{\text {vir }}(\mathfrak{M})$ for $K^{1}(\mathfrak{M})$.

This terminology is used in analogy with that of [10]. In Section 4.2 we check that our notion of virtually indecomposable agrees with that of [ibid.] in a special case.

\subsection{Epsilon functions}

We will prove that replacing direct sum decompositions by filtrations, in the formula

$$
\pi_{k}=\sum_{n \geq k} \frac{s(n, k)}{n !} E_{n},
$$

will give rise to an operator mapping $K(\mathfrak{M})$ into $K^{\leq k}(\mathfrak{M})$. In particular, we will be able to construct virtually indecomposable stack functions as 'Hall algebra logarithms'.

Fix an algebraic substack $\mathfrak{N} \hookrightarrow \mathfrak{M}$, with the following properties: 
(i) $\mathfrak{N}$ avoids the image of $\operatorname{Spec} R \stackrel{0}{\longrightarrow} \mathfrak{M}$.

(ii) $\mathfrak{N}$ is closed under direct sums and direct summands, i.e., it is Karoubian (Remark 1.63) if we add $\operatorname{Spec} R \stackrel{0}{\longrightarrow} \mathfrak{M}$ to it,

(iii) for every positive integer $n$, the morphism $\left.b\right|_{\mathfrak{N}^{(n)}}: \mathfrak{N}^{(n)} \rightarrow \mathfrak{M}$, illustrated in the diagram

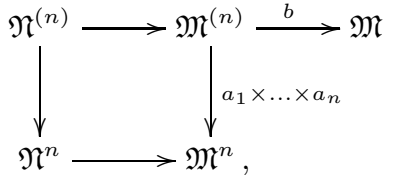

where the square is cartesian, is of finite type.

(iv) the disjoint union over all these morphisms $\coprod_{n>0} \mathfrak{N}^{(n)} \rightarrow \mathfrak{M}$ is still of finite type. This means that if $X \rightarrow \mathfrak{M}$ is a morphism with $X$ of finite type, there exists an $N>0$, such that for all $n \geq N$, the image of $\left.b\right|_{\mathfrak{N}^{(n)}}: \mathfrak{N}^{(n)} \rightarrow \mathfrak{M}$ does not intersect the image of $X$ in $\mathfrak{M}$.

Example 3.11. If $\mathfrak{M}$ is the stack of coherent sheaves on a projective curve, then the substack of non-zero semi-stable vector bundles of fixed slope is an example of a substack $\mathfrak{N}$ satisfying our conditions. More generally, we can take for $\mathfrak{N}$ the stack of all vector bundles whose HarderNarasimhan slopes are contained in a fixed interval.

Example 3.12. If $\mathfrak{M}$ is the stack of representations of a quiver $Q$, then we can take $\mathfrak{N}=\mathfrak{M}_{*}$.

Consider an arbitrary stack function $X \rightarrow \mathfrak{M}$, and denote by $F_{n} X$, for $n \geq 1$, the stack

$$
F_{n} X=\mathfrak{N}^{(n)} \times_{\mathfrak{M}} X
$$

It fits into the cartesian diagram

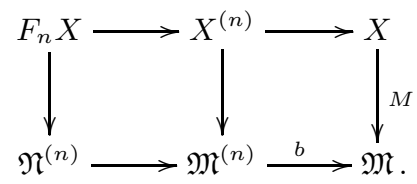

Note that $F_{n} X$ is of finite type, by our assumption on $\mathfrak{N}$, and also representable over $\mathfrak{M}$ (as an algebroid), because $b$ is. Therefore, $F_{n} X$ is another stack function.

The objects of $F_{n} X$ are pairs $(x, F)$, where $x$ is an object of $X$, and $F=\left(F_{1} \rightarrow \ldots \rightarrow F_{n}\right)$ is a flag in $F_{n}=M$, where $M$ is the image of $x$ in $\mathfrak{M}$, such that all subquotients $F_{\nu} / F_{\nu-1}$, for $\nu=1, \ldots, n$, are in $\mathfrak{N}$.

We now consider $E_{k}\left(F_{n} X\right)$, for $k \geq 0$. This is the stack of triples

$$
\left(x,\left(e_{\kappa}\right), F\right),
$$

where the pair $(x, F)$ is an object of $F_{n} X$, and $\left(e_{\kappa}\right)=\left(e_{1}, \ldots, e_{k}\right)$ is a complete set of non-zero orthogonal idempotents in $A(X)$, such that for 
every $\kappa=1, \ldots, k$ the endomorphism of $M$ induced by $e_{\kappa}$ respects the flag $F$. For every $\nu=1, \ldots, n$, we therefore get an induced idempotent operator

$$
f_{\kappa, \nu}=\left.e_{\kappa}\right|_{F_{\nu} / F_{\nu-1}} .
$$

These idempotents have the properties

(i) $\sum_{\kappa} f_{\kappa, \nu}=1$, for all $\nu$,

(ii) for every $\kappa$, at least one of the $f_{\kappa, \nu}$ does not vanish.

The stack $E_{k}\left(F_{n} X\right)$ decomposes into a disjoint union of substacks according to which of the idempotents $\left(f_{\kappa, \nu}\right)$ vanish.

To make this decomposition precise, consider a sequence of positive integers $\left(\alpha_{1}, \ldots, \alpha_{k}\right)$. Define $F_{\alpha} X$ to be the stack of triples

$$
\left(x,\left(e_{\kappa}\right),\left(F_{\kappa}\right)\right) \text {. }
$$

Here $x$ is an object of $X$, with image $M$ in $\mathfrak{M}$, and $\left(e_{\kappa}\right)$ is a complete set of orthogonal non-zero idempotents for $x$, which decomposes $M$ into a direct sum $M=\bigoplus_{\kappa} M_{\kappa}$. Moreover, $F_{\kappa}$ is a flag of length $\alpha_{\kappa}$ on $M_{\kappa}$, with subquotients in $\mathfrak{N}$, for every $\kappa=1, \ldots, k$.

For every $k$-tuple of strictly monotone maps $\Phi_{\kappa}: \underline{\alpha_{\kappa}} \hookrightarrow \underline{n}$, we define a morphism

$$
F_{\alpha} X \longrightarrow E_{k}\left(F_{n} X\right)
$$

by defining the flag $F$ on $M$ in terms of the $k$-tuple of flags $\left(F_{\kappa}\right)$ by

$$
F_{\nu}=\bigoplus_{\kappa} \sum_{\Phi_{\kappa}(\rho) \leq \nu} F_{\rho}
$$

Note that the sum for fixed $\kappa$ is not really a sum, it is just the largest of the subobjects $F_{\rho}$ of $M_{\kappa}$ making up the flag $F_{1} \rightarrow \ldots \rightarrow F_{\alpha_{\kappa}}$, such that $\Phi_{\kappa}(\rho) \leq \nu$.

Lemma 3.13. The morphism (30) given by $\left(\Phi_{\kappa}\right)_{\kappa \in \underline{k}}$ is an isomorphism onto the locus in $E_{k}\left(F_{n} X\right)$, defined by $f_{\kappa, \nu} \neq 0$ if and only if $\nu \in \Phi_{\kappa}\left(\underline{\alpha_{\kappa}}\right)$, for all $\kappa=1, \ldots, k$.

Corollary 3.14. If $\xi$ denotes the element of $K(\mathfrak{M})$ defined by $X \rightarrow \mathfrak{M}$, we have

$$
E_{k}\left(F_{n} \xi\right)=\sum_{\alpha}\left[\begin{array}{l}
n \\
\alpha
\end{array}\right] F_{\alpha}(\xi),
$$

where the sum is taken over all $k$-tuples of positive integers.

If we set $F_{0}(\xi)=1$, and $F_{\varnothing}(\xi)=1$, this equality also holds for $n=0$.

Definition 3.15. Define, for every $\xi \in K(\mathfrak{M})$,

$$
\varepsilon_{t}(\xi)=\sum_{n \geq 0}\left(\begin{array}{l}
t \\
n
\end{array}\right) F_{n}(\xi),
$$


where for $n=0$, we set $F_{0}(\xi)=1$. This definition is justified, because by our assumptions on $\mathfrak{N}$, this sum is actually finite.

Expanding in powers of $t$ defines the $\varepsilon_{k}(\xi)$, for $k \geq 0$ :

$$
\varepsilon_{t}(\xi)=\sum_{k \geq 0} \varepsilon_{k}(\xi) t^{k}
$$

For example, $\varepsilon_{0}=1$, and

$$
\varepsilon_{1}(\xi)=\sum_{n>0} \frac{(-1)^{n+1}}{n} F_{n}(\xi) .
$$

In general,

$$
\varepsilon_{k}(\xi)=\sum_{n \geq k} \frac{s(n, k)}{n !} F_{n}(\xi)
$$

Corollary 3.16. For every $k \geq 0$, we have $\varepsilon_{k}(\xi) \in K^{\leq k}(\mathfrak{M})$. Hence $\varepsilon_{t}(\xi) \in \mathscr{K}(\mathfrak{M})$. In particular, $\varepsilon_{1}(\xi)$ is virtually indecomposable, for all $\xi \in K(\mathfrak{M})$.

Proof. It suffices to prove that $E_{k}\left(\varepsilon_{t}(\xi)\right) \equiv 0 \bmod \left(t^{k}\right)$, for all $k$. In fact,

$$
\begin{aligned}
E_{k}\left(\varepsilon_{t}(\xi)\right) & =\sum_{n \geq 0}\left(\begin{array}{c}
t \\
n
\end{array}\right) E_{k} F_{n}(\xi) \\
& =\sum_{n \geq 0}\left(\begin{array}{c}
t \\
n
\end{array}\right) \sum_{\alpha_{1}, \ldots, \alpha_{k}>0}\left[\begin{array}{c}
n \\
\alpha
\end{array}\right] F_{\alpha}(\xi) \\
& =\sum_{\alpha_{1}, \ldots, \alpha_{k}>0}\left(\begin{array}{c}
t \\
\alpha_{1}
\end{array}\right) \cdots\left(\begin{array}{c}
t \\
\alpha_{k}
\end{array}\right) F_{\alpha}(\xi)
\end{aligned}
$$

is, indeed, divisible by $t^{k}$, if all $\alpha_{1}, \ldots, \alpha_{k}$ are positive.

Remark 3.17. The operator $F_{n}: K(\mathfrak{M}) \rightarrow K(\mathfrak{M})$ respects strict algebroids. The same is true for all $\varepsilon_{k}$.

\section{Epsilons as logarithms}

Suppose there exists an abelian group $\Gamma$, and a decomposition of $\mathfrak{M}$ (as an algebroid, not a linear stack) into a disjoint union

$$
\mathfrak{M}=\coprod_{\gamma \in \Gamma} \mathfrak{M}_{\gamma}
$$

We require that if $\mathfrak{E}_{\gamma, \beta}$ is defined by the cartesian diagram

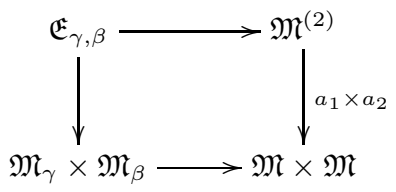


then the composition

$$
\mathfrak{E}_{\gamma, \beta} \longrightarrow \mathfrak{M}^{(2)} \stackrel{b}{\longrightarrow} \mathfrak{M}
$$

factors through $\mathfrak{M}_{\gamma+\beta} \subset \mathfrak{M}$.

We call such $\Gamma$ a grading group for $\mathfrak{M}$.

The grading group $\Gamma$ decomposes $K(\mathfrak{M})$ into a direct sum

$$
K(\mathfrak{M})=\bigoplus_{\gamma \in \Gamma} K(\mathfrak{M})_{\gamma}
$$

where $K(\mathfrak{M})_{\gamma}$ is the submodule of $K(\mathfrak{M})$ generated by stack functions $X \rightarrow \mathfrak{M}$ which factor through $\mathfrak{M}_{\gamma}$. The Hall product, as well as the commutative product, are graded with respect to (31). For $x \in K(\mathfrak{M})$, we denote the projection of $x$ into the component $K(\mathfrak{M})_{\gamma}$ by $x_{\gamma}$.

Let $\mathfrak{N} \subset \mathfrak{M}$ be a linear algebraic substack avoiding $\operatorname{Spec} R \stackrel{0}{\longrightarrow}$, with the properties

(i) every intersection $\mathfrak{N}_{\gamma}=\mathfrak{N} \cap \mathfrak{M}_{\gamma}$ is of finite type,

(ii) $\mathfrak{N}$ is closed under direct summands and extensions in $\mathfrak{M}$, the latter meaning that if $\mathfrak{N}^{(2)}$ is defined as in (29), then the composition $\mathfrak{N}^{(2)} \rightarrow \mathfrak{M}^{(2)} \stackrel{b}{\longrightarrow} \mathfrak{M}$ factors through $\mathfrak{N} \subset \mathfrak{M}$,

(iii) there is a submonoid $\Gamma_{+} \subset \Gamma$, such that $\mathfrak{N}_{\gamma} \neq \varnothing$ implies that $\gamma \in$ $\Gamma_{+} \backslash\{0\}$. The monoid $\Gamma_{+}$is required to have the property that every $\gamma \in \Gamma_{+}$admits only finitely many decompositions $\gamma=\alpha+\beta$, such that both $\alpha, \beta \in \Gamma_{+}$. We will further assume that $\Gamma_{+}$has the property that the intersection of all cofinite ideals is empty.

If these axioms hold, $\mathfrak{N}$ satisfies the finiteness conditions above, so that the $\varepsilon_{k}(\xi)$ are defined, for all $\xi \in K(\mathfrak{M})$.

Remark 3.18. If $\mathfrak{M}$ is the stack of coherent sheaves on a projective curve, then we can take $\Gamma=\mathbb{Z}^{2}$, and define $\mathfrak{M}_{(d, r)}$, for $(d, r) \in \mathbb{Z}^{2}$ to be the stack of sheaves of rank $r$ and degree $d$. Suppose $\mathfrak{N}$ is the stack of bundles whose Harder-Narasimhan slopes are contained in the interval $(a, b) \subset \mathbb{R}$. Then we can take

$$
\Gamma_{+}=\{(0,0)\} \cup\left\{(d, r) \in \mathbb{Z}^{2} \mid r>0 \text { and } a<\frac{d}{r}<b\right\},
$$

and the above requirements will be satisfied.

Remark 3.19. If $\mathfrak{M}$ is the stack of representations of a quiver $Q$, we can take $\Gamma=\mathbb{Z}^{Q_{0}}$, where $Q_{0}$ is the set of vertices of $Q$, and then set $\mathfrak{M}_{\gamma}$, for $\gamma \in \Gamma$, equal to the stack of representations with dimension vector $\gamma$. If we take $\mathfrak{N}=\mathfrak{M}_{*}$, we can take $\Gamma_{+}=\mathbb{Z}_{\geq 0}^{Q_{0}}$.

Let us also define

$$
K(\mathfrak{M})_{S}=\bigoplus_{\gamma \in S} K(\mathfrak{M})_{\gamma} \subset K(\mathfrak{M})
$$

for any cofinite ideal $S \subset \Gamma_{+}$. For every such $S$, the group $K(\mathfrak{M})_{S}$ is an ideal (with respect to both multiplications) in the ring $K(\mathfrak{M})_{+}=$ 
$K(\mathfrak{M})_{\Gamma_{+}}$, and we may complete $K(\mathfrak{M})_{+}$with respect to this collection of ideals, to obtain $\hat{K}(\mathfrak{M})_{+}$. The morphism $K(\mathfrak{M})_{+} \rightarrow \hat{K}(\mathfrak{M})_{+}$is injective and both multiplications extend to $\hat{K}(\mathfrak{M})_{+}$.

In $\hat{K}(\mathfrak{M})_{+}$the sum

$$
[\mathfrak{N}]=\sum_{\gamma \in \Gamma_{+}}\left[\mathfrak{N}_{\gamma} \rightarrow \mathfrak{M}\right]
$$

converges.

The idempotent operators commute with the $\Gamma$-grading, and so everything defined in terms of them does, too.

Proposition 3.20. In $\hat{K}(\mathfrak{M})_{+}[[t]]$, we have

$$
\varepsilon_{t}[\mathfrak{N}]=\sum_{n \geq 0}\left(\begin{array}{c}
t \\
n
\end{array}\right)[\mathfrak{N}]^{* n} .
$$

Hence we can write

$$
\varepsilon_{t}[\mathfrak{N}]=(1+[\mathfrak{N}])^{* t}=\exp _{*}\left(t \log _{*}(1+[\mathfrak{N}])\right),
$$

where exponential and logarithm are defined by their power series using the Hall product. In particular,

$$
\varepsilon_{1}[\mathfrak{N}]=\log _{*}(1+[\mathfrak{N}]),
$$

and

$$
\varepsilon_{k}[\mathfrak{N}]=\frac{1}{k !} \log (1+[\mathfrak{N}])^{* k}=\frac{1}{k !}\left(\varepsilon_{1}[\mathfrak{N}]\right)^{* k} .
$$

Proof. Using the formula

$$
F_{n}[\mathfrak{N}]=\left[\mathfrak{N}^{(n)} \stackrel{b}{\longrightarrow} \mathfrak{M}\right]=\underbrace{[\mathfrak{N}] * \ldots *[\mathfrak{N}]}_{n \text { times }},
$$

the result follows.

Remark 3.21. Compare the two formulas

$$
\begin{aligned}
\varepsilon_{t}[\mathfrak{N}] & =(1+[\mathfrak{N}])^{* t}, \\
\pi_{t}[\mathfrak{N}] & =(1+[\mathfrak{N}])^{t}
\end{aligned}
$$

Remark 3.22. Let us write $\hat{\mathscr{K}}(\mathfrak{M})_{+}$for the subspace of $\hat{K}(\mathfrak{M})_{+}[[t]]$, defined by requiring the coefficient of $t^{k}$ to be contained in $\hat{K}^{\leq k}(\mathfrak{M})_{+}$, for all $k$. Then

$$
\varepsilon_{t}[\mathfrak{N}]=(1+[\mathfrak{N}])^{* t} \in \hat{\mathscr{K}}(\mathfrak{M})_{+} .
$$

Remark 3.23. Setting $t=1$, we also get that $1+[\mathfrak{N}]=\exp _{*}\left(\varepsilon_{1}[\mathfrak{N}]\right)$. One should think of $1+[\mathfrak{N}]$ as group-like. 


\section{Hopf algebra}

We make a brief remark, without striving for generality.

Let us fix $\mathfrak{N} \subset \mathfrak{M}$ and $\Gamma_{+} \subset \Gamma$ as before. Assume for simplicity that $\Gamma$ is free. In particular, $\alpha+\beta=0$, for $\alpha, \beta \in \Gamma_{+}$, implies $\alpha=\beta=0$.

For $0 \neq \gamma \in \Gamma_{+}$, abbreviate the element $\left[\mathfrak{N}_{\gamma} \rightarrow \mathfrak{M}\right] \in K(\mathfrak{M})$ by $[\gamma]$.

For a finite sequence $(\gamma)=\gamma_{1}, \ldots, \gamma_{n}$ of non-zero elements of $\Gamma_{+}$, write

$$
[(\gamma)]=\left[\gamma_{1}, \ldots, \gamma_{n}\right]=\left[\gamma_{1}\right] * \ldots *\left[\gamma_{n}\right] .
$$

In particular, for $n=0$, we have $[\varnothing]=1$.

In many cases of interest, the Hall algebra elements $[(\gamma)]$, as $(\gamma)$ runs over all finite sequences of non-zero elements of $\Gamma_{+}$are linearly independent over $\mathbb{Q}$. Let us assume that this is the case. Then the $\mathbb{Q}$-span of all $[(\gamma)]$ is a $\mathbb{Q}$-subalgebra of $K(\mathfrak{M})$, which is free on the generators $[\gamma]$, for $\gamma \in \Gamma_{+} \backslash\{0\}$, as a unitary $\mathbb{Q}$-algebra. Let us denote this algebra by $U$. Let us further assume that the morphism induced by the commutative product $U \otimes U \rightarrow K(\mathfrak{M})$ is injective. (Again, this will hold in many cases of interest.)

We will now define a comultiplication $\Delta$ on $U$, making a $U$ a cocommutative Hopf algebra over $\mathbb{Q}$.

To define $\Delta$, it is convenient to extend the notation $[(\gamma)]$ to finite sequences of elements of $\Gamma_{+}$, which may be zero. This is done by setting $[0]=1$. Thus $[(\gamma)]$ is unchanged by 'crossing off its zeros'. We then define

$$
\begin{aligned}
\Delta: U & \longrightarrow U \otimes U \\
{[(\gamma)] } & \longmapsto \sum_{(\alpha)+(\beta)=(\gamma)}[(\alpha)] \otimes[(\beta)],
\end{aligned}
$$

where the sum is over all pairs of sequences of the same length as $\gamma$, but allowing zeros.

This defines on $U$ the structure of a cocommutative Hopf-algebra.

Lemma 3.24. The diagram

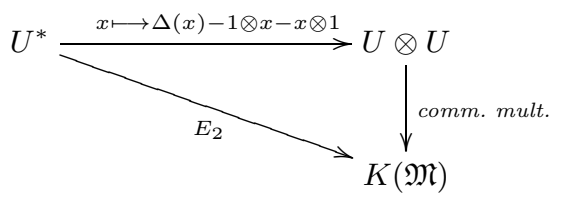

is commutative, where $U^{*} \subset U$ is the augmentation ideal.

It follows that the virtual indecomposables in $U$ are equal to the primitives with respect to the Hopf algebra structure:

$$
U^{\text {prim }}=U^{\text {vir }} .
$$

As $U$ is a cocommutative Hopf algebra, it is isomorphic to the universal enveloping algebra of $U^{\text {prim }}$, by the Cartier-Gabriel theorem [6, Theorem 3.8.2]. 
The Lie algebra $U^{\text {vir }}$ is free, as a Lie algebra over $\mathbb{Q}$, on the elements

$$
\varepsilon[\gamma]=\sum_{n>0} \frac{(-1)^{n+1}}{n} \sum_{\substack{\gamma=\gamma_{1}+\ldots+\gamma_{n} \\ \gamma_{1}, \ldots, \gamma_{n}>0}}\left[\gamma_{1}\right] * \ldots *\left[\gamma_{n}\right],
$$

for $\gamma \in \Gamma_{+} \backslash\{0\}$.

Remark 3.25. Suppose $\mathfrak{N}=\mathfrak{M}=\mathfrak{V e c t}$ is the stack of vector bundles. We take $\Gamma=\mathbb{Z}$ and $\Gamma_{+}=\mathbb{Z}_{\geq 0}$. Then the $[(\gamma)]$ are, indeed, linearly independent over $\mathbb{Q}$, at least if our ground ring $R$ is a field. Moreover, $U \otimes U \rightarrow K(\mathfrak{M})$ is injective. The Hopf algebra we obtain is the Hopf algebra of non-commutative symmetric functions, see [6, Example $4.1(\mathrm{~F})]$.

Remark 3.26. It is doubtful that it is possible to extend the coproduct to all of $K(\mathfrak{M})$, in such a way that $K^{\text {vir }}(\mathfrak{M})=K(\mathfrak{M})^{\text {prim }}$. By the above considerations, we consider the family of operators $\left(E_{n}\right)$ as a substitute, which allows us to prove at least some of the result one would expect in a cocommutative Hopf algebra. In particular, we find it unlikely that, in general, $K(\mathfrak{M})$ would be isomorphic to the universal enveloping algebra of $K^{\text {vir }}(\mathfrak{M})$. 


\section{Integration}

The integral of a stack function $(X, A) \rightarrow(\mathfrak{M}, \mathfrak{A})$ does three things: it forgets the structure map to $(\mathfrak{M}, \mathfrak{A})$, it forgets the algebroid structure, mapping $(X, A)$ to $X$, and it introduces the bundle relations in $K(\mathrm{St})$, for non-inert morphisms of algebraic stacks.

\section{The vector bundle relations}

Let $K(\mathrm{St})$ be the Grothendieck $K(\mathrm{DM})$-algebra of algebraic stacks (finite type, with affine diagonal), modulo the scissor and the bundle relations. A bundle relation is any equation of the form

$$
[Y]=[F \times X],
$$

for a fibre bundle $Y \rightarrow X$ of algebraic stacks with special structure group and fibre $F$.

It is well-known, that

$$
\begin{aligned}
K(\mathrm{St}) & =K(\mathrm{DM})\left[\frac{1}{q}\right]\left[\frac{1}{q^{n}-1}\right]_{n \geq 1} \\
& =K(\operatorname{Var})\left[\frac{1}{q}\right]\left[\frac{1}{q^{n}-1}\right]_{n \geq 1} .
\end{aligned}
$$

We prefer the latter expression in terms of $K$ (Var).

Note that the (connected, semi-simple) inertia operator does not preserve non-inert bundle relations. Therefore, in $K(\mathrm{St})$, we cannot talk about $I^{\circ, s s}[X]$, only about $\left[I_{X}^{\circ, s s}\right]$.

\section{Regular motivic weights}

Definition 4.1. We call an element of $K(\mathrm{St})$ regular, if it can be written with a denominator which does not vanish at $q=1$. Thus the subalgebra of regular motivic weights $K(\mathrm{St})_{\text {reg }} \subset K(\mathrm{St})$ is by definition the image of the morphism of $K$ (Var)-algebras

$$
K(\operatorname{Var})\left[\frac{1}{q}\right]\left[\frac{1}{q^{n}+\ldots+1}\right]_{n \geq 1} \longrightarrow K(\operatorname{Var})\left[\frac{1}{q}\right]\left[\frac{1}{q^{n}-1}\right]_{n \geq 1}=K(\mathrm{St}) .
$$

The image of $K(\mathrm{DM})$ in $K(\mathrm{St})$ is contained in $K(\mathrm{St})_{\text {reg. }}$ Hence we can also think of $K(\mathrm{St})_{r e g}$ as a $K(\mathrm{DM})$-algebra. This follows from the following Lemma.

Lemma 4.2. Every finite type stack with quasi-finite stabilizer has regular motivic weight in $K(\mathrm{St})$.

Proof. Let $Z$ be a stack with quasi-finite stabilizer (meaning that its inertia $I_{Z}$ is quasi-finite over $Z$ ). By stratifying $Z$, if necessary, we may assume that the inertia stack of $Z$ is in fact finite. By [14, Proposition 3.5.7, $Z$ is stratified by global quotient stacks, so we may assume that $Z=Y / \mathrm{GL}_{m}$, for an algebraic space $Y$, such that $\mathrm{GL}_{m}$ acts on $Y$ with finite stabilizer. The maximal torus $T \subset \mathrm{GL}_{m}$ then also acts with finite stabilizer on $Y$. The flattening stratification $\amalg Y_{i} \rightarrow Y$ of the stabilizer $\operatorname{Stab}_{T} Y$ is then $T$-equivariant, so that $T$ acts on each $Y_{i}$, and by 
passing to open and closed subspaces of the $Y_{i}$, we may assume that the action of $T$ on $Y_{i}$ has constant stabilizer (see [17, I, 5.4]). Then $T$ acts on $Y_{i}$ freely through a quotient $T_{i}$ by a finite subgroup. We conclude:

$$
[Z]=\frac{1}{\left[\mathrm{GL}_{m}\right]} \sum_{i}\left[Y_{i}\right]=\frac{1}{\left[\mathrm{GL}_{m}\right]} \sum_{i}\left[T_{i}\right]\left[Y_{i} / T_{i}\right]=\frac{[T]}{\left[\mathrm{GL}_{m}\right]} \sum_{i}\left[Y_{i} / T_{i}\right],
$$

because each quotient $T_{i}$ of $T$ is isomorphic to $T$. The last term in (32) is regular, because each $Y_{i} / T_{i}$ is an algebraic space, and

$$
\frac{[T]}{\left[\mathrm{GL}_{m}\right]}=q^{-\frac{1}{2} m(m-1)} \prod_{i=1}^{m} \frac{1}{q^{i}+\ldots+1}
$$

does not vanish at $q=1$.

\section{The integral}

Mapping a stack function $(X, A) \rightarrow(\mathfrak{M}, \mathfrak{A})$ to the class $[X] \in K(\mathrm{St})$ gives rise to a well-defined homomorphism $K(\mathfrak{M}) \rightarrow K(\mathrm{St})$ of $K(\mathrm{DM})$-modules. We denote this homomorphism by

$$
\int: K(\mathfrak{M}) \rightarrow K(\mathrm{St})
$$

\subsection{The No Poles theorem}

Theorem 4.3. The composition $\int \circ I^{\circ, \text { ss }}$ factors through the algebra of regular motivic weights:

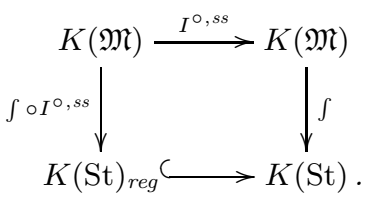

Proof. It suffices to prove that $\left[A^{\times, s s}\right] \in K(\mathrm{St})$ is regular, for every clear algebroid $(X, A)$, admitting a faithful representation. This will suffice, by Proposition 1.66. If $(X, A)$ is such an algebroid, there exists a DeligneMumford stack $Y$, with a left $\mathrm{GL}_{n}$-action, together with a strict $\mathrm{GL}_{n^{-}}$ equivariant algebra subbundle $\left.B \hookrightarrow M_{n \times n}\right|_{Y}$, such that $B^{\times}=\operatorname{Stab}_{\mathrm{GL}_{n}} Y$, and $(X, A)=\left(\mathrm{GL}_{n} \backslash Y, \mathrm{GL}_{n} \backslash B\right)$.

Let $D_{n} \subset M_{n \times n}$ be the diagonal subalgebra, and $T=D_{n}^{\times}$the standard maximal torus of $\mathrm{GL}_{n}$. As $\mathrm{GL}_{n}$ acts on $\left.B \subset M_{n \times n}\right|_{Y}$, so does the torus $T$. We will now stratify $B$ by the stabilizer with respect to the action of $T$. For this stratification to be canonical, we need $\operatorname{Stab}_{T} B$ to be the units in a finite type algebra over $B$.

In fact, such an algebra $\left.C \subset D_{n}\right|_{B}$ is given as the intersection of $\left.D_{n}\right|_{B}$ with $\left(\left.B\right|_{B}\right)^{f i x}$ inside $\left.M_{n \times n}\right|_{B}$. Here $\left(\left.B\right|_{B}\right)^{f i x}$ is the centralizer of the tautological section of $\left.B\right|_{B}$, or, under the identification $\left.B\right|_{B}=B \times_{Y} B$, the stack of commuting pairs. Thus, a section $\left.(u, b, y) \in D_{n}\right|_{B}$ is in $C$, if and only if $u \in Z_{B(y)}(b)$. 
We have, indeed, an equality

$$
C^{\times}=\operatorname{Stab}_{T} B
$$

of relative group schemes over $B$, because for $t \in T$, and $(b, y) \in B \subset$ $\left.M_{n \times n}\right|_{Y}$

$$
\begin{aligned}
t \in \operatorname{Stab}_{T}(b, y) & \Longleftrightarrow{ }^{t} b=b \text { and } t y=y \\
& \Longleftrightarrow t b=b t \text { and } t \in \operatorname{Stab}_{\mathrm{GL}_{n}(y)} \\
& \Longleftrightarrow t b=b t \text { and } t \in B^{\times}(y) \\
& \Longleftrightarrow t \in Z_{B^{\times}(y)}(b) \\
& \Longleftrightarrow t \in C^{\times}(b, y) .
\end{aligned}
$$

The subalgebras of $D_{n}$ are in one-to-one correspondence with partitions $I=\left\{I_{1}, \ldots, I_{r}\right\}$ of the set $\underline{n}=\{1, \ldots, n\}$. The partition $\underline{n}=$ $I_{1} \sqcup \ldots \sqcup I_{r}$ corresponds to the subalgebra $D_{I}$ whose primitive idempotents are the $e_{I_{\rho}}=\sum_{i \in I_{\rho}} e_{i}$, for $\rho=1, \ldots, r$. Let us write $T_{I}=D_{I}^{\times}$for the torus of units in $D_{I}$.

Now there is a unique stratification

$$
\coprod_{I} B_{I} \longrightarrow B
$$

such that a section $(b, y)$ of $B$ factors through $B_{I}$, if and only if the pullback of $\left.C \subset D_{n}\right|_{B}$ via $(b, y)$ is equal to $D_{I}$. The existence of this stratification is proved by passing to the rank stratification (see Definition 1.23) of $C$, and observing that a subalgebra bundle $\left.C \subset D_{n}\right|_{S}$, for any stack $S$, decomposes $S$ into a disjoint union of open and closed substacks, such that $C$ is constant over these components. We may reformulate the defining property of $B_{I}$ by saying that $(b, y) \in B_{I}$, if and only if $Z_{B(y)}(b) \cap D_{n}=D_{I}$. We also have, for $(b, y) \in B_{I}$, that $\operatorname{Stab}_{T}(b, y)=C^{\times}(b, y)=D_{I}^{\times}=T_{I}$.

The stratification (34) is $T$-equivariant, because for $t \in T$, and $(b, y) \in$ $B$, we have

$$
\begin{aligned}
(b, y) \in B_{I} & \Longleftrightarrow Z_{B(y)}(b) \cap D_{n}=D_{I} \\
& \Longleftrightarrow Z_{B(t y)}\left({ }^{t} b\right) \cap{ }^{t} D_{n}={ }^{t} D_{I} \\
& \Longleftrightarrow Z_{B(t y)}\left({ }^{t} b\right) \cap D_{n}=D_{I} \\
& \Longleftrightarrow\left({ }^{t} b, t y\right) \in B_{I},
\end{aligned}
$$

as $T \subset D_{n}$, and $D_{n}$ is commutative.

(Let us remark that we were not able to prove that for a general action of $T$ on a Deligne-Mumford stack $Y$, the stabilizer stratifies $Y$ equivariantly. The fact that the stabilizer is equal to the units in an algebra helps. Note also, that we did not prove a defining property for (34) in terms of stabilizers in T.)

So, for every partition $I$ of $\underline{n}$, the torus $T$ acts on $B_{I} \subset B$, with stabilizer $T_{I}$. We therefore get an induced action of $T / T_{I}$ on $B_{I}$. Matrix conjugation preserves units, so we get and induced action of $T / T_{I}$ on

$$
B_{I}^{\times}=B_{I} \cap B^{\times} .
$$


In fact, this action even respects $B_{I}^{\times, s s}=B_{I} \cap B^{\times, s s}$, but the following modification does not.

Consider the action of $T_{I} \subset T$ on $B_{I}$ by left multiplication:

$$
t(b, y)=(t b, y) .
$$

This is a well-defined action, because $t \in T_{I}$, and $(b, y) \in B_{I}$, implies that $t \in Z_{B(y)}(b)$. In particular, $t \in B(y)$, so that $(t, y) \in B$, and the product $(t, y)(b, y)=(t b, y)$ in $B$ exists. Moreover, $(t b, y) \in B_{I}$, because $Z_{B(y)}(t b) \cap D_{n}=Z_{B(y)}(b) \cap D_{n}$. This action of $T_{I}$ on $B_{I}$ preserves $B_{I}^{\times}$. Over fields, it also preserves sections which are semi-simple, because the product of two semi-simple commuting matrices is again a semi-simple matrix. (Note that this does not imply that $T_{I}$ acts on $B_{I}^{s s}$, because even if $(b, y)$ is a strict section of $B$, the product $(t b, y)$ may not be strict.)

We finally consider the action of $T(I)=T_{I} \times T / T_{I}$ on $B_{I}^{\times}$, defined by

$$
\left(t^{\prime}, t\right) *(b, y)=\left(t^{\prime t} b, t y\right) .
$$

The quotient stack $Z_{I}=B_{I}^{\times} / T(I)$ is a finite type scheme over $R$, so its Zariski topological space $\left|Z_{I}\right|$ is a Zariski space (see [15], Chapter 5). By Chevalley's theorem (see [ibid.]), the image of $\left|B_{I}^{\times, s s}\right|$ in $\left|Z_{I}\right|$ is constructible, so we can find disjoint, locally closed (reduced) algebraic substacks $Z_{1}, \ldots, Z_{n} \subset Z_{I}$, such that this image is equal to $\left|Z_{1}\right| \sqcup \ldots \sqcup\left|Z_{n}\right| \subset\left|Z_{I}\right|$. Let

$$
\widetilde{Z}_{I}=Z_{1} \amalg \ldots \amalg Z_{n} .
$$

This is a finite type algebraic stack endowed with a representable monomorphism $\widetilde{Z}_{I} \rightarrow Z_{I}$.

We claim that $\widetilde{Z}_{I}$ is an algebraic stack with quasi-finite stabilizer. This will follow from the fact that, for field valued points, the action of $T(I)$ on $B_{I}^{\times, s s}$ has finite stabilizers. To see this, assume that

$$
\left(t^{\prime} b, t y\right)=(b, y)
$$

for $\left(t^{\prime}, t\right) \in T_{I} \times T / T_{I}$, and $(b, y) \in B_{I}^{\times, s s}$. This implies that $t y=y$, hence conjugation by $t$ preserves the fiber $B(y)$ of $B$ over $y$. We have

$$
t^{\prime t} b=b \text {, }
$$

where $t^{\prime}$ commutes with $b$. Rewriting as ${ }^{t} b=t^{\prime-1} b$, we see that ${ }^{t} b$ commutes with $b$. Changing basis, if necessary, we can diagonalize the three matrices $b,{ }^{t} b$ and $t^{\prime}$, simultaneously. Since $b$ and ${ }^{t} b$ have the same eigenvalues, we see that the entries of the diagonal matrix ${ }^{t} b$, are obtained from those of $b$ by a permutation. Hence there are at most $n$ ! possible values for ${ }^{t} b$, and hence for $t^{\prime}=b^{t} b^{-1}$. For every one of these possible values of $t^{\prime}$, there is at most one $t \in T / T_{I}$, such that ${ }^{t} b=t^{\prime-1} b$. Thus the action (35) has finite stabilizers, at least on field valued points of $B_{I}^{\times, s s}$, as required.

Consider the cartesian diagram

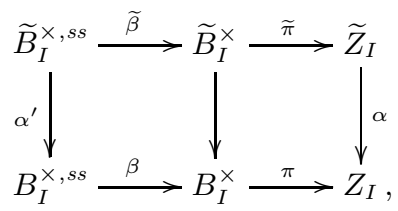


obtained by pulling back $B_{I}^{\times, s s} \rightarrow B_{I}^{\times} \rightarrow Z_{I}$ via $\widetilde{Z}_{I} \rightarrow Z_{I}$. The morphisms $\alpha$ and $\beta$ are disjoint unions of isomorphisms onto locally closed substacks, so the same is true for $\alpha^{\prime}$ and $\widetilde{\beta}$. But both $\alpha^{\prime}$ and $\widetilde{\beta}$ are surjective on underlying Zariski topological spaces, so by the scissor relations, we have

$$
\left[B_{I}^{\times, s s}\right]=\left[\widetilde{B}_{I}^{\times, s s}\right]=\left[\widetilde{B}_{I}^{\times}\right],
$$

in $K(\mathrm{St})$. The morphism $\pi$ is a principal $T(I)$-bundle, so the same is true for $\tilde{\pi}$, and so by the bundle relations, we have

$$
\left[B_{I}^{\times, s s}\right]=\left[\widetilde{B}_{I}^{\times}\right]=[T(I)]\left[\widetilde{Z}_{I}\right]=(q-1)^{n}\left[\widetilde{Z}_{I}\right],
$$

in $K(\mathrm{St})$. It follows, that we have

$$
\begin{aligned}
{\left[A^{\times, s s}\right] } & =\left[\mathrm{GL}_{n} \backslash B^{\times, s s}\right]=\frac{\left[B^{\times, s s}\right]}{\left[\mathrm{GL}_{n}\right]}=\frac{1}{\left[\mathrm{GL}_{n}\right]} \sum_{I}\left[B_{I}^{\times, s s}\right] \\
& =\frac{1}{\left[\mathrm{GL}_{n}\right]} \sum_{I}(q-1)^{n}\left[\widetilde{Z}_{I}\right]=q^{-\frac{1}{2} n(n-1)} \prod_{i=1}^{n-1} \frac{1}{q^{i}+\ldots+1} \sum_{I}\left[\widetilde{Z}_{I}\right] .
\end{aligned}
$$

The claim now follows from Lemma 4.2

Corollary 4.4. The multiple $(q-1)^{k} \int$ of the integral takes regular values on $K^{\leq k}(\mathfrak{M})$, for every $k \geq 0$.

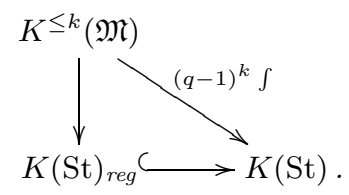

Proof. Consider the following diagram:

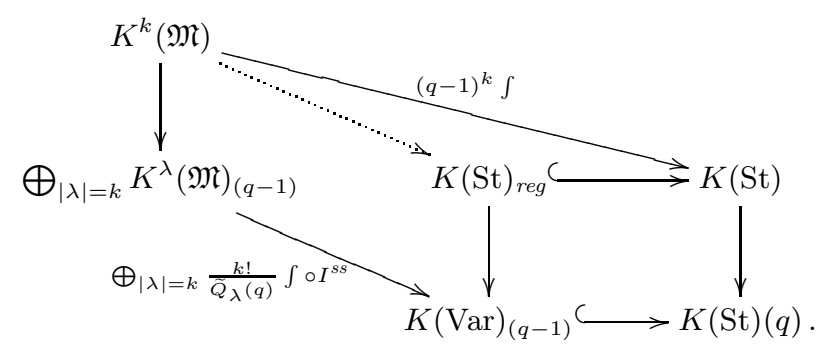

The dotted arrow exists because the square in the lower right of this diagram is cartesian, and the outer part of the diagram commutes. (Here we have identified the localization $K(\operatorname{Var})_{(q-1)}$ with its image in $K(\mathrm{St})(q)=$ $\left.K(\mathrm{St}) \otimes_{\mathbb{Q}[q]} \mathbb{Q}(q).\right)$

Corollary 4.5. Defining $\int t=q-1$ extends the integral to a $K$ (Var)linear homomorphism

$$
\int: \mathscr{K}(\mathfrak{M}) \longrightarrow K(\mathrm{St})_{\text {reg }}
$$




\subsection{The integral vs. the Hall product}

\section{The $\Gamma$-indexed integral}

Let $\Gamma$ be a grading group for $\mathfrak{M}$, as in Section 3.3 We assume, in addition, that $\Gamma$ is endowed with a $\mathbb{Z}$-valued bilinear form $\chi$.

Definition 4.6. We call $\mathfrak{M}$ hereditary, if for every $\gamma, \beta \in \Gamma$, the morphism $\mathfrak{E}_{\gamma, \beta} \rightarrow \mathfrak{M}_{\gamma} \times \mathfrak{M}_{\beta}$ is a vector bundle stack ([4, Definition 1.9) of $\operatorname{rank}-\chi(\beta, \gamma)$.

Let us assume henceforth that $\mathfrak{M}$ is hereditary.

We define $K(\mathrm{St})[\Gamma]$ to be the free $K(\mathrm{St})$-module on the symbols $u^{\gamma}$, for $\gamma \in \Gamma$, and introduce an associative product on $K(\mathrm{St})[\Gamma]$ by the formula

$$
u^{\gamma} * u^{\beta}=q^{-\chi(\beta, \gamma)} u^{\gamma+\beta},
$$

and extend it linearly, to make $K(\mathrm{St})[\Gamma]$ a $K(\mathrm{St})$-algebra. Regular coefficients form a subalgebra $K(\mathrm{St})_{\text {reg }}[\Gamma]$.

We define the $\Gamma$-indexed integral

$$
\begin{gathered}
\int: \mathscr{K}(\mathfrak{M}) \longrightarrow K(\mathrm{St})_{\text {reg }}[\Gamma] \\
\sum_{\gamma \in \Gamma} x_{\gamma} \longmapsto \sum_{\gamma \in \Gamma} u^{\gamma} \int x_{\gamma} .
\end{gathered}
$$

Proposition 4.7. If $\mathfrak{M}$ is hereditary the $\Gamma$-indexed integral preserves the star product. In fact, for $x, y \in \mathscr{K}(\mathfrak{M})$ we have

$$
\int x * y=\int x * \int y \quad \in K(\mathrm{St})_{\text {reg }}[\Gamma]
$$

Proof. This is a straightforward calculation. One uses the fact that for $X \rightarrow \mathfrak{M}_{\gamma}$ and $Y \rightarrow \mathfrak{M}_{\beta}$, the morphism $X * Y \rightarrow X \times Y$ is a vector bundle stack of rank $-\chi(\gamma, \beta)$, and hence, in $K(\mathrm{St})$, we have $[X * Y]=$ $q^{-\chi(\gamma, \beta)}[X][Y]$.

\section{Semi-classical limit}

We will pass to the semi-classical limit of the integral $\int: \mathscr{K}(\mathfrak{M}) \rightarrow$ $K(\mathrm{St})_{\text {reg }}[\Gamma]$, by setting $t=0$ (in the source), and hence $q=1$ (in the target). As $\int$ respects the $*$-product, the semi-classical limit will be a morphism of Poisson algebras.

Modulo $(q-1)$, the star product on $K(\mathrm{St})_{r e g}[\Gamma]$ is commutative, in fact, modulo $(q-1)$ it is given by the commutative product $u^{\gamma} \cdot u^{\beta}=u^{\gamma+\beta}$. Hence the quotient $K(\mathrm{St})_{r e g}[\Gamma] /(q-1)$ inherits a Poisson bracket, defined by

$$
x * y-y * x \equiv\{x, y\}(q-1) \quad \bmod (q-1)^{2} .
$$

Explicitly, it is given by

$$
\left\{u^{\gamma}, u^{\beta}\right\}=-\tilde{\chi}(\beta, \gamma) u^{\gamma+\beta}
$$


where $\tilde{\chi}$ is (twice) the anti-symmetrization of $\chi$ :

$$
\widetilde{\chi}(\beta, \gamma)=\chi(\beta, \gamma)-\chi(\gamma, \beta)
$$

We conclude:

Theorem 4.8. If $\mathfrak{M}$ is hereditary, we have a morphism of Poisson algebras

$$
\int_{q=1}: K(\mathfrak{M}) \longrightarrow K(\mathrm{St})_{r e g} /(q-1)[\Gamma]
$$

The Poisson structure on $K(\mathfrak{M})$ is described in Section [3.2, the one on $K(\mathrm{St})_{\text {reg }} /(q-1)[\Gamma]$, above, see (37). The $u^{\gamma}$ coefficient of the integral $\int_{q=1}$ may be expressed as

$$
\int_{q=1} x=\left.\left(\int \pi_{q-1}(x)\right)\right|_{q=1}=\left.\sum_{n=0}^{\infty}\left(\begin{array}{c}
q-1 \\
n
\end{array}\right) \int E_{n}(x)\right|_{q=1},
$$

for $x \in K(\mathfrak{M})_{\gamma}$. Here we have used the operator $\pi_{t}$ of Definition 2.10 , and substituted $t=q-1$.

Proof. The homomorphism (38) is obtained by setting $t=0$ in (36). Note that the deformation parameter $t$ is mapped to the deformation parameter $(q-1)$, so that the Poisson bracket (which depends on the choice of the deformation parameter) is preserved.

To calculate $\int_{q=1}$, note that $x \mapsto \pi_{t}(x)$ is a section of the quotient map $\mathscr{K}(\mathfrak{M}) \rightarrow K(\mathfrak{M})$, obtained by setting $t=0$. This gives rise to displayed formula.

Remark 4.9. Note that the diagram

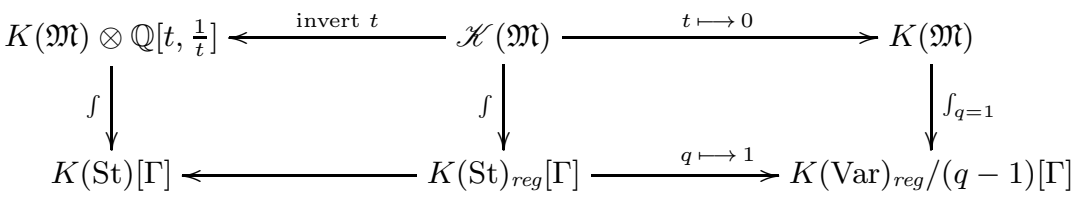

commutes. The central column is a morphism of one-parameter families of non-commutative algebras. The left hand column is the general fibre, and a morphism of non-commutative $K(\operatorname{Var}) \otimes \mathbb{Q}\left[t, \frac{1}{t}\right]$-algebras, the right hand column is the semi-classical limit, and hence a morphism of Poisson algebras.

Restricting the theorem to the virtually indecomposable elements, we obtain:

Corollary 4.10. The semi-classical limit of the integral defines a morphism of Lie algebras over K(Var):

$$
\int_{q=1}: K^{\text {vir }}(\mathfrak{M}) \longrightarrow K(\mathrm{St})_{\text {reg }} /(q-1)[\Gamma] .
$$


The bracket in $K^{\text {vir }}(\mathfrak{M})$ is the commutator bracket of the Hall product, the bracket in $K(\operatorname{Var})_{\text {reg }} /(q-1)[\Gamma]$ is given in 37$)$. The integral $\int_{q=1}$ is given by the formula

$$
\int_{q=1} x=\left.\sum_{\gamma \in \Gamma} u^{\gamma}\left((q-1) \int x_{\gamma}\right)\right|_{q=1}
$$

for a virtually indecomposable Hall algebra element $x \in K^{\text {vir }}(\mathfrak{M})$.

Remark 4.11. We have a surjective morphism of $K$ (Var)-algebras

$$
K(\operatorname{Var}) /(q-1) \stackrel{\sim}{\longrightarrow} K(\mathrm{St})_{r e g} /(q-1) .
$$

This morphism is (most likely), not injective, because there is no (obvious) reason why elements in $\operatorname{Ann}(q-1) \subset K$ (Var) should map to zero in $K(\operatorname{Var}) /(q-1)$, although they certainly map to zero in $K(\mathrm{St})_{\text {reg }} /(q-1)$.

Without too much more effort, it is possible to prove that the semiclassical limit of the integral lifts to a $K$ (Var)-linear homomorphism

$$
\int_{q=1}: \mathscr{K}(\mathfrak{M}) \longrightarrow K(\operatorname{Var}) /(q-1)[\Gamma]
$$

Unfortunately, we cannot, at the moment, prove that this lift is a morphism of Poisson algebras.

Remark 4.12. We leave it to the (interested) reader to write down the analogue of Theorem 4.8 for the case that $\mathfrak{M}$ is Calabi-Yau-3, rather than hereditary. This will include proving our main theorems for an equivariant version $K(\mathfrak{M})^{\hat{\mu}}$ of $K(\mathfrak{M})$, and including vanishing cycle and orientation data weights in the integral. 


\section{Appendix. Comparison with Joyce's vir- tual projections in an example}

Let us write $[n]=\left[B \mathrm{GL}_{n} \rightarrow \mathfrak{V} \mathfrak{e c t}\right] \in K(\mathfrak{V} \mathfrak{e c t})$. We have (cf. Remark 3.21)

$$
E_{r}[n]=\sum_{\substack{\ell_{1}+\ldots+\ell_{r}=n \\ \ell_{1} \ldots \ell_{r}>0}}\left[\ell_{1}\right] \ldots\left[\ell_{r}\right] .
$$

This gives us

$$
\begin{aligned}
\pi_{k}[n] & =\sum_{r} \frac{s(r, k)}{r !} E_{r}[n] \\
& =\sum_{r} \frac{s(r, k)}{r !} \sum_{\substack{\ell_{1}+\ldots+\ell_{r}=n \\
\ell_{1} \ldots \ell_{r}>0}}\left[\ell_{1}\right] \ldots\left[\ell_{r}\right] \\
& =\sum_{\lambda \vdash n} \frac{s(|\lambda|, k)}{|\operatorname{Aut} \lambda|} \prod_{i}\left[\lambda_{i}\right] .
\end{aligned}
$$

We remark also, that the formula of Remark 2.11 gives us

$$
\pi_{t}\left(\sum_{n \geq 0}[n] u^{n}\right)=\left(\sum_{n \geq 0}[n] u^{n}\right)^{t}
$$

which contains the above formulas for $\pi_{k}[n]$.

In [11, §5.2], Joyce defines projection operators $\Pi_{n}^{v i}: K(\mathfrak{M}) \rightarrow K(\mathfrak{M})$, which pairwise commute, and add up to the identity (although he works with bare algebraic stacks, not algebroids). We expect that modulo this difference, we have

$$
\pi_{k}=\Pi_{k}^{v i}
$$

We will prove that these operators take the same values on the elements $[n] \in K(\mathfrak{V e c t})$.

Proposition .13. We have

$$
\pi_{k}[n]=\Pi_{k}^{v i}[n]
$$

for all $k$ and $n$.

Proof. Let $T_{n}$ be the $n$-dimensional torus of diagonal matrices inside $\mathrm{GL}_{n}$.

Joyce's $\mathcal{P}$ set [11, Definition 5.3] is trivial in this case because as a quotient stack $\mathrm{BGL}_{n}=* / \mathrm{GL}_{n}$ where $*$ is a point so $\mathcal{P}\left(*, T_{n}\right)=\left\{T_{n}\right\}$. The $\mathcal{Q}$ set $\mathcal{Q}\left(\mathrm{GL}_{n}, T_{n}\right)$, is computed in [11, Example 5.7] to be the set of all tori

$$
T_{\varphi}:=\left\{\operatorname{diag}\left(z_{1}, \cdots, z_{n}\right): z_{i} \in \mathbb{G}_{m}, z_{i}=z_{j} \text { if } \varphi(i)=\varphi(j), \quad \forall i, j\right\} .
$$

where $\varphi$ ranges over all surjection maps $\varphi: \underline{n} \rightarrow \underline{r}$. Finally the $\mathcal{R}$ set coincides with $\mathcal{Q}$. Joyce's definition then needs computation of $M_{G}^{X}(P, Q, R)$ 
where $P, Q$, and $R$ are selected respectively from $\mathcal{P}, \mathcal{Q}$ and $\mathcal{R}$. In our case this is

$$
M_{\mathrm{GL}_{n}}^{*}\left(T_{n}, Q, R\right)=\left|\frac{N_{\mathrm{GL}_{n}}\left(T_{n}\right)}{C_{\mathrm{GL}_{n}}(Q) \cap N_{\mathrm{GL}_{n}}\left(T_{n}\right)}\right|^{-1} n_{T_{n}}^{\mathrm{GL}_{n}}(R, Q)
$$

for all choices of $R, Q \in \mathcal{Q}\left(\mathrm{GL}_{n}, T_{n}\right)$ such that $R \subseteq Q$. Now we unwind the definition of $n_{T_{n}}^{\mathrm{GL}_{n}}(R, Q)$.

$$
n(R, Q)=\sum_{\substack{B \subseteq\{\hat{Q} \in \mathcal{Q}: \hat{Q} \subseteq Q\} \\ Q \in B, \cap_{\hat{Q} \in B} \hat{Q}=R}}(-1)^{|B|-1}
$$

We can finally define the virtual projections of $\mathrm{BGL}_{n}$ as

$$
\Pi_{k}^{v i}\left(\mathrm{BGL}_{n}\right)=\sum_{R: \operatorname{dim} R=k} \sum_{Q: R \subseteq Q} M_{\mathrm{GL}_{n}}^{*}\left(T_{n}, Q, R\right)\left[B C_{G}(Q)\right] .
$$

We say $Q \in \mathcal{Q}\left(\mathrm{GL}_{n}, T_{n}\right)$ is of 'type $\sigma$ ' if the corresponding surjection $\varphi:\{1, \cdots, n\} \rightarrow\{1, \cdots, r\}$ induces the partition $\sigma \vdash n$. Note that, there are $\frac{n !}{\sigma_{1} ! \cdots \sigma_{n} !(1 !)^{\sigma_{1} \cdots(n !)^{\sigma_{n}}}}$ of them. Also $C_{\mathrm{GL}_{n}} Q$ only depends on the type of $Q$ and is isomorphic to $\prod_{i=1}^{n}\left[\mathrm{GL}_{i}\right]^{\sigma_{i}}$. The normalizer of $T_{n}$ is $S_{n} \ltimes T^{n}$ and therefore

$$
C_{\mathrm{GL}_{n}}\left(T_{\varphi}\right) \cap N_{\mathrm{GL}_{n}}\left(T_{n}\right)=\prod_{i=1}^{n}\left[S_{i} \ltimes T_{i}\right]^{\sigma_{i}}
$$

and

$$
\left|\frac{N_{\mathrm{GL}_{n}}\left(T^{n}\right)}{C_{\mathrm{GL}_{n}}(Q) \cap N_{\mathrm{GL}_{n}}\left(T_{n}\right)}\right|^{-1}=\frac{(1 !)^{\sigma_{1}} \cdots(n !)^{\sigma_{n}}}{n !} .
$$

We have

$$
\begin{aligned}
\Pi_{k}^{v i}\left(\mathrm{BGL}_{n}\right) & =\sum_{R: \operatorname{dim} R=k} \sum_{Q: R \subseteq Q} M_{\mathrm{GL}_{n}}^{*}\left(T_{n}, Q, R\right)\left[B C_{G}(Q)\right] \\
& =\sum_{Q}\left|\frac{N_{\mathrm{GL}_{n}}\left(T_{n}\right)}{C_{\mathrm{GL}_{n}}(Q) \cap N_{\mathrm{GL}_{n}}\left(T_{n}\right)}\right|^{-1}\left(\sum_{\substack{R \subseteq Q \\
\operatorname{dim} R=k}} n_{T_{n}}^{\mathrm{GL}_{n}}(R, Q)\right)\left[B C_{G}(Q)\right] \\
& =\sum_{\sigma}(\# Q \text { of type } \sigma) \cdot \frac{(1 !)^{\sigma_{1}} \cdots(n !)^{\sigma_{n}}}{n !} s(|\sigma|, k) \prod_{i=1}^{n}\left[\mathrm{BGL}_{i}\right]^{\sigma_{i}} \\
& =\sum_{\sigma} \frac{1}{\sigma_{1} ! \cdots \sigma_{n} !} s(|\sigma|, k) \prod_{i=1}^{n}\left[\mathrm{BGL}_{i}\right]^{\sigma_{i}} .
\end{aligned}
$$

where the third line follow from the lemma below. We conclude that Joyce's virtual projections of $\mathrm{BGL}_{n}$ are identical to our eigenprojections.

Lemma .14. For a $Q$ of type $\sigma$, we have

$$
\sum_{R: \operatorname{dim} R=k} n(R, Q)=s(|\sigma|, k) .
$$


Proof. We let $m=\operatorname{dim} Q=|\sigma|$ in this proof. Obviously if $|\sigma|<k$, there is no possible choice of

$$
B \subseteq\{\hat{Q}: \hat{Q} \subseteq Q\}: Q \in B, \bigcap_{\hat{Q} \in B} \hat{Q}=R
$$

therefore proving

$$
\sum_{R: \operatorname{dim} R=k} n(R, Q)=s(|\sigma|, k) \quad \text { if } \quad|\sigma|<k .
$$

In the case that $|\sigma|=k$, the only choice of $R$ is $Q$ itself and the only choice of $B$ is the set $B=\{Q\}$. This proves

$$
\sum_{R: \operatorname{dim} R=k} n(R, Q)=s(|\sigma|, k) \quad \text { if } \quad|\sigma|=k .
$$

All other values of $s(|\sigma|, k)$ are defined recursively by

$$
s(m, k)=s(m-1, k-1)-(m-1) s(m-1, k) .
$$

So it suffices to show that $\sum_{R: \operatorname{dim} R=k} n(R, Q)$ satisfies the same recursive relation.

For any choice of $R$,

$$
n(R, Q)=\sum_{\substack{B \subseteq\{\hat{Q} \in \mathcal{Q}: \hat{Q} \subseteq Q\} \\ Q \in B, \cap}}(-1)^{|B|-1}
$$

can be computed also form only choosing those $\hat{Q}$ that are codimension 1 inside $Q$. This is because for every $\hat{Q}$ of codimension $>2$ the number $t$ of intermediate subtori $Q^{\prime}$

$$
\hat{Q} \subset Q^{\prime} \subseteq Q
$$

is positive and therefore $B$ containing $\hat{Q}$ is included in $2^{t}$ possible choices of $B$ with cancelling size parities.

Let's write the points of $Q$ as $m$-tuples $\left(x_{1}, \ldots, x_{m}\right)$ with $x_{i} \in \mathbb{G}_{m}$. Let $W$ be the $m-1$ dimensional torus consisting of points $\left(x_{1}, \ldots, x_{m-1}\right)$. Any $R$ with $\operatorname{dim} R=k$ is given by a set of defining equations

$$
x_{i_{1}}=\cdots=x_{i_{k_{i}}}, i=1,2, \ldots
$$

In defining equation of $R$ with $\operatorname{dim} R=k$, either $x_{m}$ does not appear in which case $\left.R\right|_{W}$ is $k-1$ dimensional. The second case is if $x_{m}$ appears in defining equation of $R$, in which case $\left.R\right|_{W}$ is $k$ dimensional and any choice of $B$ consisting of only codimension 1 elements, satisfying $\bigcap_{\hat{Q} \in B} \hat{Q}=R$ loses one of its elements after restriction to $\left.R\right|_{W}$. This shows that

$$
\begin{gathered}
\sum_{\substack{B \subseteq\{\hat{Q} \in \mathcal{Q}: \hat{Q} \subseteq Q\} \\
Q \in B, \operatorname{dim} \cap \hat{Q} \in B}}(-1)^{|B|-1}=\sum_{\substack{B \subseteq\{\hat{Q} \in \mathcal{Q}: \hat{Q} \subseteq W\} \\
W \in B, \operatorname{dim} \bigcap_{\hat{Q} \in B} \hat{Q}=k-1}}(-1)^{|B|-1} \\
-(m-1) \sum_{\substack{B \subseteq\{\hat{Q} \in \mathcal{Q}: \hat{Q} \subseteq W\} \\
W \in B, \operatorname{dim} \bigcap_{\hat{Q} \in B} \hat{Q}=k}}(-1)^{|B|-1}
\end{gathered}
$$

which completes the proof. 


\section{References}

[1] D. Abramovich, M. Olsson, and A. Vistoli. Tame stacks in positive characteristic. Ann. Inst. Fourier (Grenoble), 58(4):1057-1091, 2008.

[2] M. Artin, J. E. Bertin, M. Demazure, A. Grothendieck, P. Gabriel, M. Raynaud, and J.-P. Serre. Schémas en groupes SGA3. Institut des Hautes Études Scientifiques, Paris, 1963/1966.

[3] M. Artin, A. Grothendieck, and J. L. Verdier. Théorie des Topos et Cohomologie Etale des Schémas, SGA4. Lecture Notes in Mathematics Nos. 269, 270, 305. Springer, Berlin, Heidelberg, New York, 1972,73 .

[4] K. Behrend and B. Fantechi. The intrinsic normal cone. Invent. Math., 128(1):45-88, 1997.

[5] T. Bridgeland. An introduction to motivic Hall algebras. Adv. Math., 229(1):102-138, 2012.

[6] P. Cartier. A primer of Hopf algebras. In Frontiers in number theory, physics, and geometry. II, pages 537-615. Springer, Berlin, 2007.

[7] A. D'Agnolo and P. Polesello. Deformation quantization of complex involutive submanifolds. In Noncommutative geometry and physics, pages 127-137. World Sci. Publ., Hackensack, NJ, 2005.

[8] A. Grothendieck. Revêtements etales et groupe fondamental SGA1, volume 224 of Lecture notes in mathematics. Springer-Verlag, 1971.

[9] A. Grothendieck and J. Dieudonné. Éléments de géométrie algébrique EGA. Number 4, 8, 11, 17, 20, 24, 28, and 32 in Publications Mathématiques. Institut des Hautes Études Scientifiques, 1960-1967.

[10] D. Joyce. Configurations in abelian categories. II. Ringel-Hall algebras. Adv. Math., 210(2):635-706, 2007.

[11] D. Joyce. Motivic invariants of Artin stacks and 'stack functions'. $Q$. J. Math., 58(3):345-392, 2007.

[12] D. Joyce and Y. Song. A theory of generalized Donaldson-Thomas invariants. Mem. Amer. Math. Soc., 217(1020):iv+199, 2012.

[13] M. Kontsevich. Deformation quantization of algebraic varieties. Lett. Math. Phys., 56(3):271-294, 2001. EuroConférence Moshé Flato 2000, Part III (Dijon).

[14] A. Kresch. Cycle groups for Artin stacks. Invent. Math., 138(3):495536, 1999.

[15] G. Laumon and L. Moret-Bailly. Champs algébriques, volume 39 of Ergebnisse der Mathematik und ihrer Grenzgebiete. 3. Folge. Springer-Verlag, Berlin, 2000.

[16] B. Noohi. Fundamental groups of algebraic stacks. J. Inst. Math. Jussieu, 3(1):69-103, 2004.

[17] J. Oesterlé. Schémas en groupes de type multiplicatif. In Autour des schémas en groupes. Vol. I, volume 42/43 of Panor. Synthèses, pages 63-91. Soc. Math. France, Paris, 2014. 
[18] The Stacks Project Authors. Stacks Project. http://stacks. math. columbia. edu, 2015. Last time retrieved December 2016. 